\title{
Chapter 16 Organic-Inorganic Hybrid Perovskite Solar Cells
}

\author{
Hiroyuki Fujiwara, Nikolas J. Podraza, Maria Isabel Alonso, Masato Kato, \\ Kiran Ghimire, Tetsuhiko Miyadera, Masayuki Chikamatsu
}

\begin{abstract}
Quite high efficiencies exceeding 20\% have been realized in solar cells incorporating organic-inorganic hybrid perovskites $\left(\mathrm{APbX}_{3}\right)$, which have a unique structure with a center cation $\left[\mathrm{A}=\mathrm{CH}_{3} \mathrm{NH}_{3}{ }^{+}, \mathrm{HC}\left(\mathrm{NH}_{2}\right)_{2}{ }^{+}\right]$located within a $\mathrm{PbX}_{3}{ }^{-}$cage $(\mathrm{X}=\mathrm{I}, \mathrm{Br}, \mathrm{Cl})$. Superior characteristics of hybrid perovskite solar cells can be understood from the nature of optical transitions and the efficient carrier collection in the device. From these points of view, this chapter provides details on optical properties of various hybrid perovskite materials and carrier dynamics in the solar cells. In particular, based on the first-principles analyses of different perovskite materials, we present universal rules that allow the unified interpretation of the optical absorption phenomenon in $\mathrm{APbX}_{3}$ perovskites. The external quantum efficiency (EQE) analysis further reveals that high short-circuit current densities $\left(>20 \mathrm{~mA} / \mathrm{cm}^{2}\right)$ observed in the perovskite solar cells originate from electric-field-assisted carrier collection and the suppressed optical losses in the devices. Although hybrid perovskites have quite favorable characteristics for solar cells, these materials exhibit rather intense phase change upon exposure to humid air. In this chapter, the degradation process of $\mathrm{CH}_{3} \mathrm{NH}_{3} \mathrm{PbI}_{3}$ in humid air, characterized by applying ellipsometry technique, is further presented and discussed.
\end{abstract}

H. Fujiwara, M. Kato

Gifu University, 1-1 Yanagido, Gifu 501-1193, Japan

e-mail: fujiwara@gifu-u.ac.jp

N. J. Podraza, K. Ghimire

University of Toledo, Toledo, $\mathrm{OH} 43606$, USA

\section{I. Alonso}

Institut de Ciència de Materials de Barcelona (ICMAB-CSIC), Campus UAB, 08193 Bellaterra, Spain.

T. Miyadera, M. Chikamatsu

Research Center for Photovoltaics, National Institute of Advanced Industrial Science and Technology, 1-1-1 Umezono, Tsukuba, 305-8568, Japan 


\subsection{Introduction}

Research into organic-inorganic hybrid perovskite solar cells is progressing rapidly and quite remarkable conversion efficiencies exceeding $20 \%$ have already been realized by using hybrid perovskite light absorbers [1-4]. The operation of a hybrid perovskite solar cell was first demonstrated by Kojima et al. using methylammonium lead iodide $\left(\mathrm{MAPbI}_{3}, \mathrm{CH}_{3} \mathrm{NH}_{3} \mathrm{PbI}_{3}\right)$ in 2009 [5]. Although the conversion efficiency of this solar cell was $3.8 \%$, later the efficiency was improved to $10.9 \%$ in 2012 [6], leading to drastic expansion of the researches on hybrid perovskite photovoltaic devices [7-14].

In the last few years, formamidinium lead iodide [ $\left.\mathrm{FAPbI}_{3}, \mathrm{HC}\left(\mathrm{NH}_{2}\right)_{2} \mathrm{PbI}_{3}\right]$, which exhibits higher thermal stability, has been applied to solar cells as an alternative hybrid perovskite material $[1-4,15-17]$. However, this perovskite is intrinsically unstable and a cubic $\mathrm{FAPbI}_{3}$ crystal $\left(\alpha-\mathrm{FAPbI}_{3}\right)$ shows a gradual phase transformation into a transparent $\delta$-FAPbI 3 phase having a one-dimensional crystal structure [18,19]. Quite fortunately, $\mathrm{FAPbI}_{3}$-based perovskites can be stabilized by including a small amount of $\mathrm{MA}^{+}$and $\mathrm{Cs}^{+}$and, as a result, a variety of hybrid perovskites $\left(\mathrm{APbX}_{3}\right)$ consisting of mixed center cations $\left(\mathrm{A}=\mathrm{MA}^{+}, \mathrm{FA}^{+}, \mathrm{Cs}^{+}\right)$with different $\mathrm{PbX}_{3}{ }^{-}$cages $(\mathrm{X}=\mathrm{I}, \mathrm{Br})$ have been developed [1-4,20-24]. To date, quite high conversion efficiencies over $20 \%$ have been demonstrated in (FA,MA) $\mathrm{Pb}(\mathrm{I}, \mathrm{Br})_{3}$ and (FA, MA, $\left.\mathrm{Cs}\right) \mathrm{Pb}(\mathrm{I}, \mathrm{Br})_{3}$ solar cells [1-4].

Figure 16.1 shows basic crystal structures of $\mathrm{MAPbI}_{3}$ and $\mathrm{FAPbI}_{3}$ hybrid perovskites: (a) $\mathrm{MAPbI}_{3}$ (pseudocubic), (b) $\mathrm{MAPbI}_{3}$ (tetragonal) and (c) $\alpha-\mathrm{FAPbI}_{3}$ (pseudocubic). These crystal structures represent the optimized crystal structures obtained from the density functional theory (DFT) calculation using the Perdew-Burke-Ernzerhof (PBE) scheme [25] within the cubic [Figs. 16.1(a) and (c)] and tetragonal [Fig. 16.1(b)] bases $[26,27]$. The arrows indicate the directions of the $a, b$, and $c$ axes of the unit cells. It can be seen that hybrid perovskite compounds in Fig. 16.1 have a unique combination of the organic A-site center cation with the inorganic cage consisting of $\mathrm{PbI}_{3}{ }^{-}$. At room temperature, $\mathrm{MAPbI}_{3}$ single crystals show a tetragonal structure $[9,28,29]$. However, the $\mathrm{MAPbI}_{3}$ crystals become cubic above $57{ }^{\circ} \mathrm{C}$ [9]. For $\mathrm{FAPbI}_{3}$, there are two different phase structures at room temperature (i.e., $\alpha$-FAPbI 3 and $\delta$-FAPbI 3 ) $[15,18,29]$ of which $\delta$ - $\mathrm{FAPbI}_{3}$, that will be shown later, is the most stable crystal structure at room temperature $[18,19]$.

In Figs. 16.1(a) and (c), the unit cells have pseudocubic structures with the lattice parameters of $a=6.306 \AA, b=6.291 \AA$ and $c=6.310 \AA\left(\mathrm{MAPbI}_{3}\right)$ and $a=6.416 \AA, b=$ $6.236 \AA$, and $c=6.353 \AA\left(\alpha-\mathrm{FAPbI}_{3}\right)$. Thus, the unit cell sizes of $\mathrm{MAPbI}_{3}$ and $\alpha-\mathrm{FAPbI}_{3}$ are quite similar. However, $a$ of $\alpha-\mathrm{FAPbI}_{3}$ is expanded slightly, compared with $b$ and $c$, 
(a) $\mathrm{MAPbl}_{3}$ (Pseudocubic)

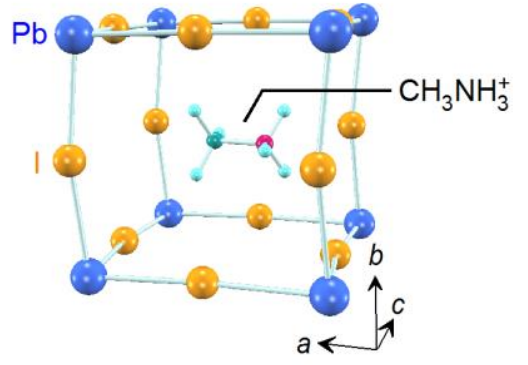

(c) $\alpha-\mathrm{FAPbl}_{3}$ (Pseudocubic)

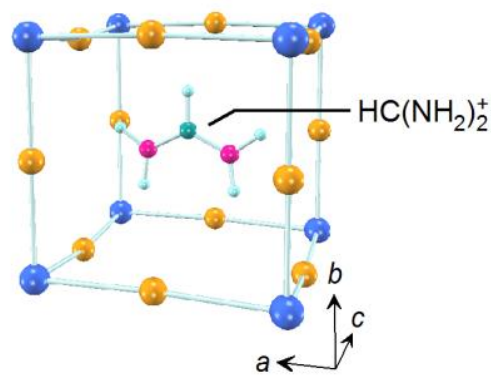

(b) $\mathrm{MAPbl}_{3}$ (Tetragonal)

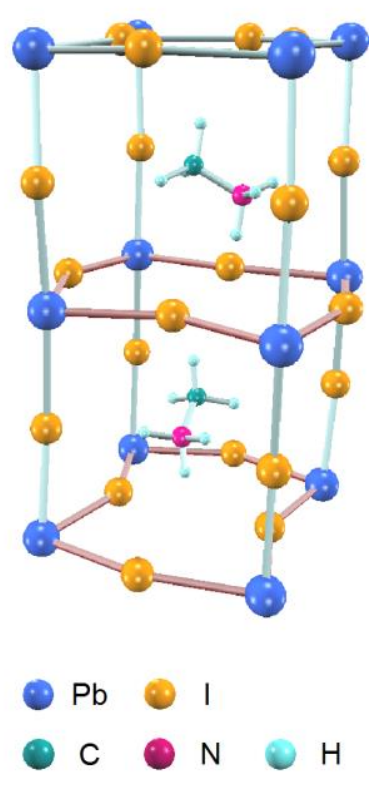

Fig. 16.1 Crystal structures of $\mathrm{MAPbI}_{3}$ and $\mathrm{FAPbI}_{3}$ hybrid perovskites determined by DFT calculations using PBE: (a) $\mathrm{MAPbI}_{3}$ (pseudocubic) [26], (b) $\mathrm{MAPbI}_{3}$ (tetragonal) and (c) $\alpha-\mathrm{FAPbI}_{3}$ (pseudocubic) [27]. The arrows indicate the $a, b$, and $c$ axes of the pseudocubic unit cells. In (b), the in-plane Pb-I networks are shown by the thin red color.

due to the steric effect of $\mathrm{FA}^{+}$, while the lattice parameters are almost the same in $\mathrm{MAPbI}_{3}$. These values are comparable to the experimental values of $a=6.259 \AA$ $\left(\mathrm{MAPbI}_{3}\right)$ [9] and $a=6.362 \AA\left(\alpha-\mathrm{FAPbI}_{3}\right)$ [19].

In the DFT-optimized structures obtained assuming $0 \mathrm{~K}, \mathrm{MA}^{+}$and $\mathrm{FA}^{+}$interact with $\mathrm{I}$ atoms, forming hydrogen bonds expressed by $\mathrm{I} \cdots \mathrm{H}-\mathrm{N}$, and the orientation and location of the center cation are determined primarily by this hydrogen bonding interaction [19,26,27,30-33]. Nevertheless, it has been confirmed experimentally that, at room temperature, $\mathrm{MA}^{+}$and $\mathrm{FA}^{+}$reorient quite rapidly (0.5-14 ps) inside the $\mathrm{Pb}$-I network [19,34-40]. In Fig. 16.1, on the other hand, the I-Pb-I angle is slightly bent and, in the tetragonal phase, the I-Pb-I bond angle changes rather significantly. It has been 


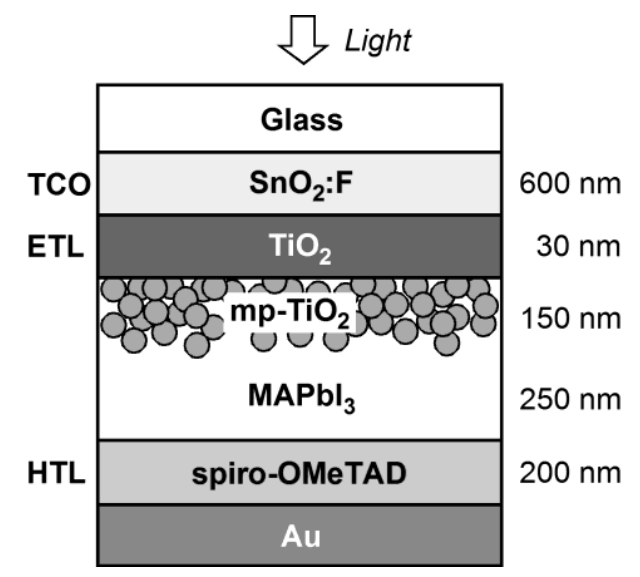

Fig. 16.2 Basic structure of a hybrid perovskite $\left(\mathrm{MAPbI}_{3}\right)$ solar cell fabricated by spin coating. In this structure, the $\mathrm{MAPbI}_{3}$ absorber layer is inserted between the mesoporous $(\mathrm{mp})-\mathrm{TiO}_{2}$ electron transport layer (ETL) and spiro-OMeTAD hole transport layer (HTL).

reported that the introduction of the large center cation leads to the formation of collinear I-Pb-I bonds, which are closer to $180^{\circ}$ [41]. Quite interestingly, the collinear bond formation induces the slight band gap $\left(E_{\mathrm{g}}\right)$ reduction $[30,41,42]$, which has been explained by the anti-bonding character of the $\mathrm{Pb}-\mathrm{I}$ bond $[41,42]$.

Figure 16.2 shows a basic structure of a hybrid perovskite $\left(\mathrm{MAPbI}_{3}\right)$ solar cell $[6,8,10,12]$. This solar cell consists of a glass substrate, $\mathrm{SnO}_{2}: \mathrm{F}$ transparent conductive oxide (TCO), $\mathrm{TiO}_{2}$ electron transport layer (ETL), light absorber $\left(\mathrm{MAPbI}_{3}\right)$, spiro-OMeTAD hole transport layer (HTL) and back metal electrode (Au or Ag). The spiro-OMeTAD is an organic compound (see Fig. 9.18 in Vol. 2), which is employed quite commonly in hybrid perovskite solar cells $[6,8]$ and can be formed by spin coating. For the fabrication of the solar cells, commercial glass substrates on which TCO $\left(\mathrm{SnO}_{2}: \mathrm{F}\right)$ layers are formed (TEC-8 or TEC-15, Pilkington) are used widely (see Chapter 18). On this $\mathrm{SnO}_{2}: \mathrm{F} /$ glass substrate, a dense (compact) $\mathrm{TiO}_{2}$ layer, followed by a porous (mesoporous; mp) $\mathrm{TiO}_{2}$ layer, is formed prior to the perovskite layer fabrication [6,8]. One quite remarkable feature of hybrid perovskite solar cells is that high efficiency devices can be made by adopting a rather simple solution-based process, combined with low temperature annealing at $\sim 100{ }^{\circ} \mathrm{C}[6,7,12]$. Accordingly, by further developing hybrid perovskite solar cells, low-cost production of large-area solar cell modules could be realized. 
(a)

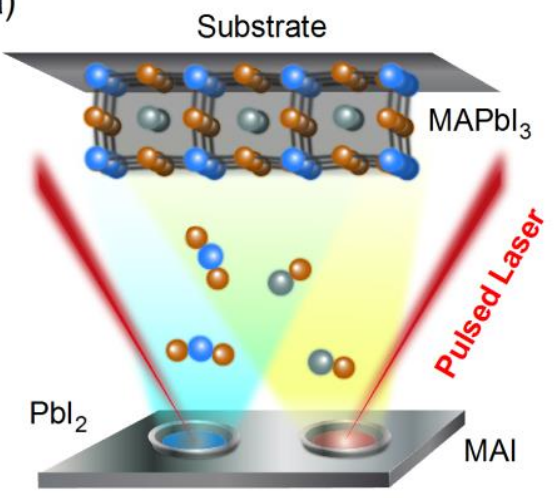

(b)

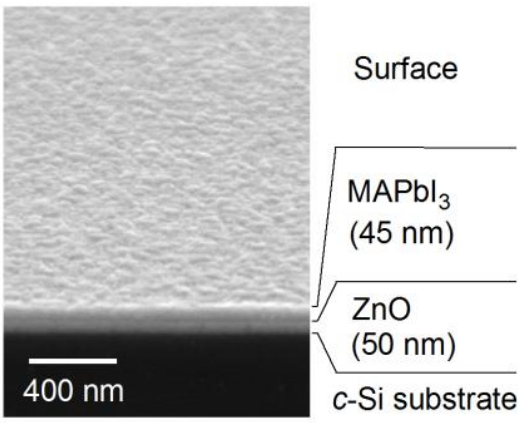

Fig. 16.3 (a) Schematic of the laser evaporation process and (b) a scanning electron microscope (SEM) image of the $\mathrm{MAPbI}_{3} / \mathrm{ZnO} / c$-Si structure [26].

In this chapter, we will overview the optical characteristics of unique hybrid perovskite compounds and the operational principles of the solar cells (Sections 16.2 16.3, H. Fujiwara, M. Kato, T. Miyadera and M. Chikamatsu). Moreover, this chapter describes spectroscopic ellipsometry (SE) characterization of hybrid perovskite structures based on real-time measurements (Section 16.4, N. J. Podoraza and K. Ghimire). One of the drawbacks of hybrid perovskite solar cells is a rather strong degradation in humid air, and the degradation phenomenon and its mechanism are discussed in Section 16.5 (M. I. Alonso).

\subsection{Optical Properties}

\subsubsection{Optical Constants of Hybrid Perovskites}

For $\mathrm{MAPbI}_{3}$, many inconsistent optical data have been reported [26,43-52] (see Fig. 1.3). However, the disagreement observed among the reported optical constants can primarily be attributed to the influence of roughness component (Chapter 6). Since spectroscopic ellipsometry (SE) is a surface-sensitive technique, the fabrication of samples with smooth surfaces is essential for reliable SE characterization. For the preparation of smooth $\mathrm{MAPbI}_{3}$ and $\alpha-\mathrm{FAPbI}_{3}$ layers, a laser evaporation technique shown in Fig. 16.3(a) has been applied [26,53]. In this technique, $\mathrm{PbI}_{2}$ and MAI (or FAI) source materials are heated by a near-infrared laser, and the evaporation rates of the source materials are controlled precisely by adjusting the pulsed-laser power and 
frequency. By using this technique, an ultrasmooth $\mathrm{MAPbI}_{3}$ layer shown in Fig. 16.3(b) can be formed [26] and, in this sample, a thin $\mathrm{ZnO}$ layer is provided to improve film adhesion on a crystalline $\mathrm{Si}(c-\mathrm{Si})$ substrate. For the suppression of surface roughening and structural non-uniformity in the growth direction, characterization of thin layers [45 $\mathrm{nm}$ in Fig. 16.3(b)] is quite effective (Chapter 6). The $\mathrm{MAPbI}_{3}$ and $\alpha-\mathrm{FAPbI}_{3}$ layers fabricated by laser evaporation show sharp x-ray diffraction peaks, which are consistent with the perovskite pseudocubic phases, and the formation of secondary $\mathrm{PbI}_{2}$ phase is negligible [26,27].

Unfortunately, $\mathrm{MAPbI}_{3}$ [51,54-59] and $\alpha$-FAPbI 3 [16,21,23,29] exhibit significant degradation in humid air (Section 16.5). Thus, to avoid the air exposure, the samples were transferred from the deposition system to the SE measurement system using a $\mathrm{N}_{2}$-filled plastic bag. From this procedure, the SE spectra of the pristine $\mathrm{MAPbI}_{3}$ and $\alpha$-FAPbI ${ }_{3}$ were measured without exposing the samples to humid air at all $[26,27]$, although the high-energy spectra of $\mathrm{MAPbI}_{3}(E \geq 4.75 \mathrm{eV})$ were obtained after the short-time air exposure ( 20 s) at 40\% relative humidity [26].

Moreover, to determine reliable optical constants of hybrid perovskites, the SE analyses have been performed using a global error minimization (GEM) scheme [26,27], in which the dielectric function is determined self-consistently using more than two samples having different layer thicknesses on substrates [60,61] (Section 10.2). In this method, the dielectric function is obtained first from a thin layer and the extracted dielectric function is then applied for the SE analysis of a thicker layer (or layers) under the assumption that the bulk-layer optical properties are independent of the layer thickness. In the actual GEM analyses of the hybrid perovskites, a pair of samples with thicknesses in a range of 45-200 $\mathrm{nm}$ have been employed [26,27].

Figure 16.4 shows an example of GEM analysis performed for $\mathrm{MAPbI}_{3}$ [26]. In this case, two samples with $\mathrm{MAPbI}_{3}$ layer thicknesses of $45 \mathrm{~nm}$ [Fig. 16.3(b)] and $85 \mathrm{~nm}$ were used and two sets of ellipsometry spectra were obtained from these samples [i.e., $(\psi, \Delta)_{45 \mathrm{~nm}}$ and $\left.(\psi, \Delta)_{85 \mathrm{~nm}}\right]$. For this SE analysis, an optical model shown in Fig. 16.4(a) has been assumed. The interface and $\mathrm{SiO}_{2}$ layers correspond to the surface roughness of the $\mathrm{ZnO}$ layer and the native oxide of the $c$-Si substrate, respectively. The $\mathrm{ZnO}$ surface roughness can be characterized from the $\mathrm{SE}$ analysis of a $\mathrm{ZnO} / \mathrm{SiO}_{2} / c-\mathrm{Si}$ structure (see Section 18.3.2). The optical properties of the surface roughness layer are calculated as a 50:50 vol.\% mixture of the bulk layer and voids by applying the Bruggeman effective-medium approximation (EMA) [60,61] (see Sections 3.4.2 and 6.1), while a 50:50 vol.\% mixture of $\mathrm{MAPbI}_{3}$ and $\mathrm{ZnO}$ is assumed for the interface layer.

Figure 16.4(b) shows experimental SE spectra of $(\psi, \Delta)_{85 \mathrm{~nm}}$ and the solid lines 
(a)

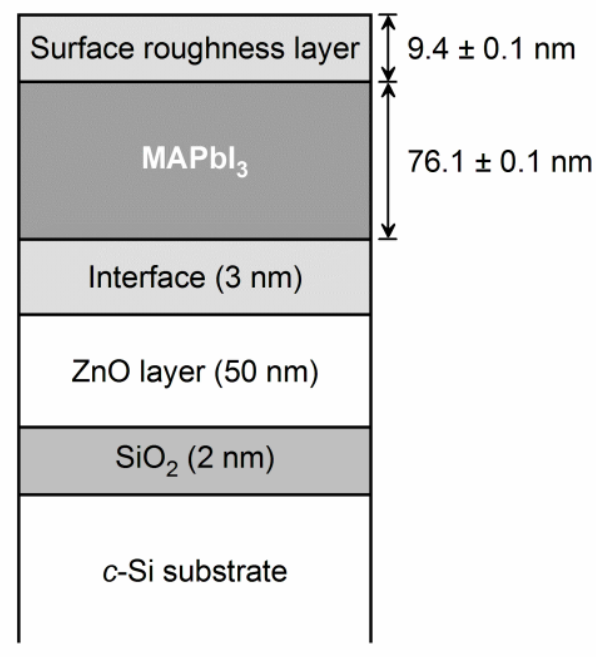

(b)

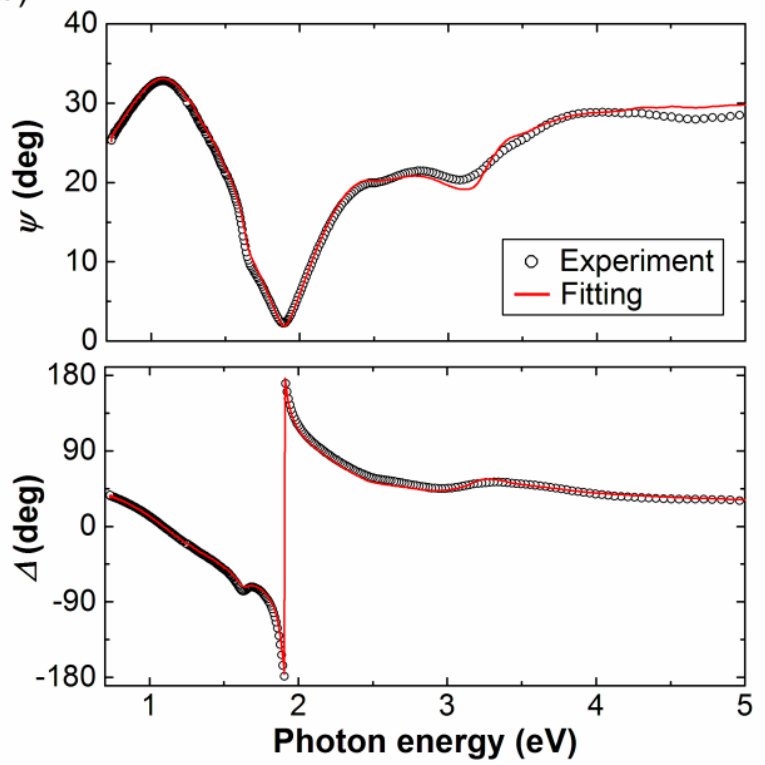

Fig. 16.4 (a) Optical model and (b) $(\psi, \Delta)$ ellipsometry spectra for a $\mathrm{MAPbI}_{3}(85$ $\mathrm{nm}) / \mathrm{ZnO}(50 \mathrm{~nm}) / \mathrm{SiO}_{2}(2 \mathrm{~nm}) / c$-Si structure [26]. The solid lines show the fitting result calculated using the $\mathrm{MAPbI}_{3}$ dielectric function extracted from the thinner layer (45 nm). In (a), the layer thicknesses determined from the SE analysis of (b) are shown. In (b), the SE measurement was carried out in a $\mathrm{N}_{2}$ environment without exposing the sample to air at an angle of incidence of $75^{\circ}$.

represent the fitting result calculated using the $\mathrm{MAPbI}_{3}$ dielectric function extracted from $(\psi, \Delta)_{45 \mathrm{~nm}}[26]$. The calculated spectra show excellent fitting to the experimental spectra, confirming the validity of the analysis. The layer thicknesses determined from the SE analysis of Fig. 16.4(b) are also summarized in Fig. 16.4(a). In the subsequent analysis, the final dielectric function is extracted from $(\psi, \Delta)_{45 \mathrm{~nm}}$ by adjusting the $\mathrm{MAPbI}_{3}$ bulk layer thickness slightly since the $\varepsilon_{2}$ values obtained from the above analysis show very small negative values $\left(\varepsilon_{2} \sim-0.05\right)$ at $E<E_{\mathrm{g}}$. In particular, the bulk layer thickness is increased slightly ( $\sim 5 \%$ of total thickness) so that the $\varepsilon_{2}$ values at $E<$ $E_{\mathrm{g}}$ become completely zero. Such minor adjustment is often necessary due to the imperfection of samples. It should be noted that the surface-roughness layer thickness of $2.9 \pm 0.1 \mathrm{~nm}$ obtained from the SE analysis of $(\psi, \Delta)_{45 \mathrm{~nm}}$, shows reasonable agreement with the root-mean-square roughness of $4.6 \mathrm{~nm}$ observed in AFM. Thus, the overall ellipsometry analysis can be justified by comparing the roughness values characterized from SE and AFM [Fig. 6.1(b)]. It has been confirmed that thermal annealing $\left(80^{\circ} \mathrm{C}\right.$ for 
(a)

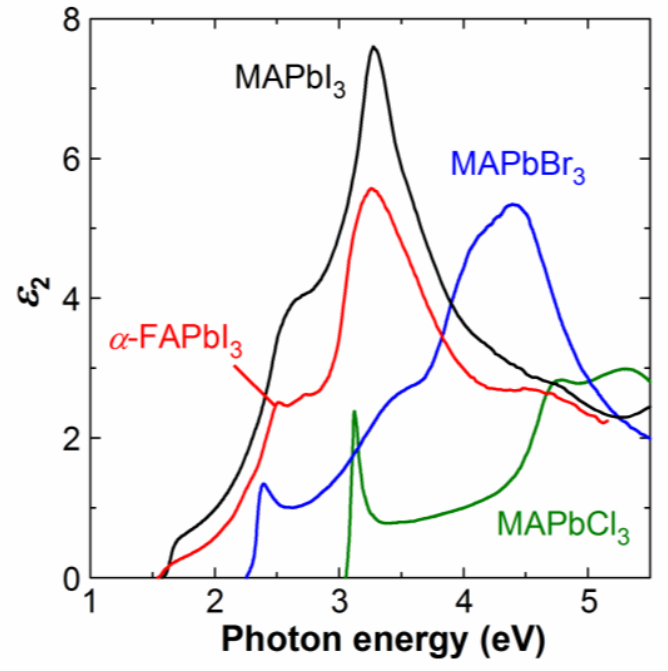

(b)

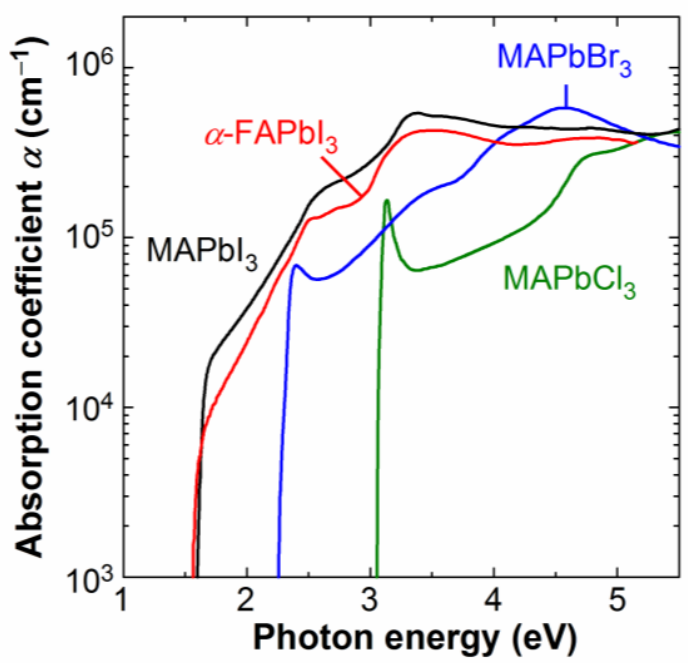

Fig. 16.5 (a) $\varepsilon_{2}$ spectra and (b) $\alpha$ spectra of $\mathrm{MAPbX}_{3}(\mathrm{X}=\mathrm{I}, \mathrm{Br}, \mathrm{Cl})$ and $\alpha-\mathrm{FAPbI}_{3}$. The reported optical data of $\mathrm{MAPbI}_{3}$ [26], $\alpha$ - $\mathrm{FAPbI}_{3}$ [27], $\mathrm{MAPbBr}_{3}$ [52], and $\mathrm{MAPbCl}_{3}$ [52] are shown. The results of $\alpha-\mathrm{FAPbI}_{3}$ and $\mathrm{MAPbI}_{3}$ were obtained from the GEM analyses of polycrystalline layers, whereas those of $\mathrm{MAPbBr}_{3}$ and $\mathrm{MAPbCl}_{3}$ were extracted from the single crystals.

45 min) of a $\mathrm{MAPbI}_{3}$ sample does not change the SE result significantly, while annealing at a higher temperature $\left(100{ }^{\circ} \mathrm{C}\right.$ for $\left.45 \mathrm{~min}\right)$ leads to $\mathrm{PbI}_{2}$ formation by the MAI desorption [26].

Figure 16.5 summarizes (a) the $\varepsilon_{2}$ spectra and (b) the $\alpha$ spectra of $\mathrm{MAPbX}_{3}$ and $\alpha-\mathrm{FAPbI}_{3}$. The optical functions of $\mathrm{MAPbI}_{3}$ [26] and $\alpha-\mathrm{FAPbI}_{3}$ [27] have been determined from the GEM analyses of the laser-evaporated layers (i.e., Fig. 16.4 for $\mathrm{MAPbI}_{3}$ ), whereas those of $\mathrm{MAPbBr}_{3}$ and $\mathrm{MAPbCl}_{3}$ have been obtained from the SE analyses of the single crystals [52]. The complete optical data of these materials are also shown in Chapter 10 (Vol. 2). In Fig. 16.5(a), when the X-site halogen atom is changed, the whole dielectric function shifts toward higher energy with the gradual reduction of the $\varepsilon_{2}$ amplitude for the lighter halogen atom. In this case, a high-energy transition peak observed at $3.24 \mathrm{eV}$ in $\mathrm{MAPbI}_{3}$ splits into two peaks in $\mathrm{MAPbBr}_{3}$ and $\mathrm{MAPbCl}_{3}$. The sharp absorption peaks of $\mathrm{MAPbBr}_{3}$ and $\mathrm{MAPbCl}_{3}$, observed near the $E_{\mathrm{g}}$ regions, originate from excitonic transitions [62] and the excitonic peaks become more pronounced in $\mathrm{MAPbCl}_{3}$.

Quite interestingly, the $\varepsilon_{2}$ amplitude of $\alpha-\mathrm{FAPbI}_{3}$ is roughly half of that of $\mathrm{MAPbI}_{3}$ at $E<3 \mathrm{eV}$, even though the overall $\varepsilon_{2}$-spectral shape is independent of the center cation. 
Accordingly, the replacement of the A-site cation has a large influence on the absorption strength. In fact, the $\alpha$ values of $\alpha-\mathrm{FAPbI}_{3}$ are notably smaller than those of $\mathrm{MAPbI}_{3}$ and, at $1.7 \mathrm{eV}, \alpha$ of $\alpha-\mathrm{FAPbI}_{3}$ is half of that of $\mathrm{MAPbI}_{3}$ [Fig. 16.5(b)]. The lower light absorption observed in $\alpha$-FAPbI 3 explains why thicker absorber layers $(\sim 500 \mathrm{~nm})$ are generally necessary for $\mathrm{FAPbI}_{3}$-based perovskite solar cells [1-4], compared with $\mathrm{MAPbI}_{3}$ solar cells (typically $\sim 300 \mathrm{~nm}$ ) [7]. The low $\alpha$ values observed in $\alpha-\mathrm{FAPbI}_{3}$ have also been confirmed based on external quantum efficiency (EQE) analysis of a $\alpha-\mathrm{FAPbI}_{3}$ solar cell [27]. The results of Fig. 16.5 show clearly that the A-site cation $\left(\mathrm{MA}^{+}\right.$and $\left.\mathrm{FA}^{+}\right)$modifies the oscillator strength of the optical transition (or $\varepsilon_{2}$ amplitude), while the $\mathrm{X}$-site halogen atom determines the transition energy.

Often, high conversion efficiencies demonstrated in $\mathrm{MAPbI}_{3}$ solar cells are attributed to quite high $\alpha$ in $\mathrm{MAPbI}_{3}[13,63,64]$. Nevertheless, many $\alpha$ values reported earlier for $\mathrm{MAPbI}_{3}$ have been overestimated seriously due to the influence of surface roughness (see Fig. 1.3 and Chapter 6), and the actual $\alpha$ values of $\mathrm{MAPbI}_{3}$ in Fig. 16.5(b) are comparable to those of $\mathrm{CuInGaSe}_{2}, \mathrm{GaAs}$, and CdTe absorbers (Fig. 1.6). In other words, the $\alpha$ values of $\alpha-\mathrm{FAPbI}_{3}$ are lower than major solar-cell materials. Since hybrid perovskite layers fabricated by spin-coating processes are generally thin $(<1 \mu \mathrm{m})$, the optical confinement is of significant importance particularly in $\mathrm{FAPbI}_{3}$-based solar cells (see also Section 16.3).

Figure 16.6 summarizes (a) the dielectric functions and (b) the $\alpha$ spectra of hybrid-perovskite secondary phases including $\mathrm{PbI}_{2}$ [26], MAI [26] and $\delta$-FAPbI ${ }_{3}$ [27], together with those of $\mathrm{MAPbI}_{3}$ and $\alpha-\mathrm{FAPbI}_{3}$ in Fig. 16.5. As confirmed from Fig. 16.6, $\mathrm{PbI}_{2}$ exhibits $\varepsilon_{2}$ peaks at 2.51, 2.97, 3.26, 3.90, and $4.33 \mathrm{eV}$, where the rather sharp peak at $2.51 \mathrm{eV}$ has been attributed to an excitonic transition [65,66]. On the other hand, the visible light absorption in MAI and $\delta$-FAPbI 3 is quite weak.

Figure 16.7 presents the critical point (CP) analyses of (a) $\mathrm{MAPbI}_{3}$ [26] and (b) $\alpha-\mathrm{FAPbI}_{3}$ [27] dielectric functions. As described in Section 4.3.1, the CP analysis is used commonly to determine the optical transition energies and $E_{\mathrm{g}}$ accurately. For the $\mathrm{CP}$ analyses of $\mathrm{MAPbI}_{3}$ in Fig. 16.7(a) and $\alpha-\mathrm{FAPbI}_{3}$ in Fig. 16.7(b), a procedure described in Section 10.3.2 has been employed. Briefly, in this CP analysis, the second-derivative spectra calculated from the modeled dielectric functions (Chapter 10 in Vol. 2) are employed to suppress the spectral noise and the theoretical fitting to these spectra is then carried out. The open circles in Figs. 16.7(a) and (b) show the $\mathrm{d}^{2} \varepsilon_{1} / \mathrm{d} E^{2}$ spectrum obtained from the modeled dielectric functions, while the solid lines represent the fitting results calculated from Eqs. (10.2) and (10.3). From the CP analysis, $E_{0}\left(E_{\mathrm{g}}\right)$ of $\mathrm{MAPbI}_{3}$ is found to be $1.61 \pm 0.01 \mathrm{eV}$, while the peak transition energies in the high 
(a)

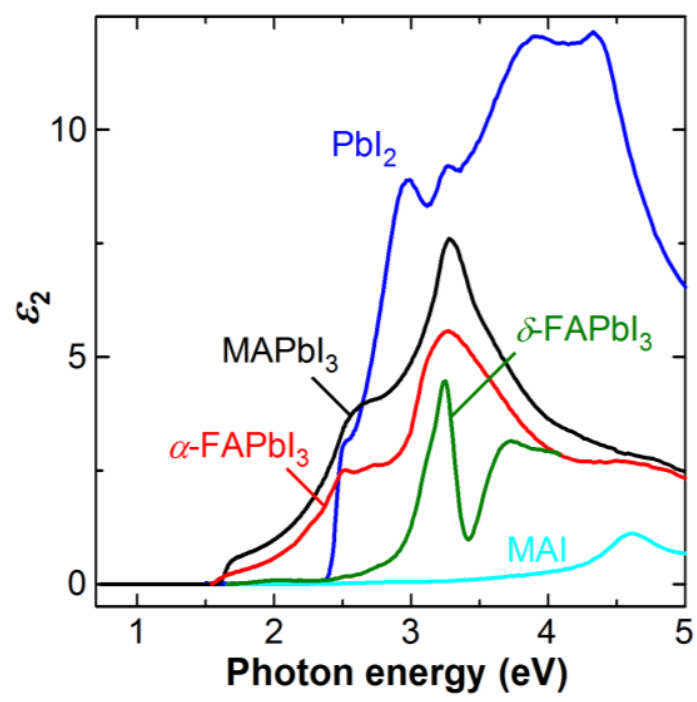

(b)

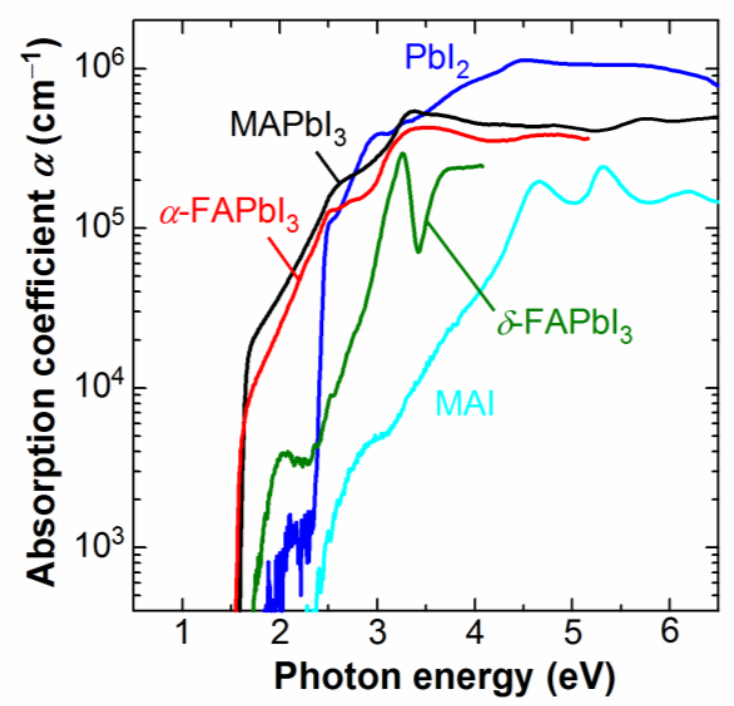

Fig. 16.6 (a) $\varepsilon_{2}$ spectra and (b) $\alpha$ spectra of hybrid-perovskite secondary phases including $\mathrm{PbI}_{2}$ [26], MAI [26] and $\delta-\mathrm{FAPbI}_{3}$ [27], together with those of $\mathrm{MAPbI}_{3}$ and $\alpha-\mathrm{FAPbI}_{3}$ in Fig. 16.5.

energy region are determined to be $E_{1}=2.53 \pm 0.01 \mathrm{eV}$ and $E_{2}=3.24 \pm 0.01 \mathrm{eV}$ [26]. The $\mathrm{CP}$ analysis of $\alpha-\mathrm{FAPbI}_{3}$ has been implemented assuming only three transitions at $E$ $\leq 3.5 \mathrm{eV}$, which results in the CP energies of $1.55 \pm 0.01 \mathrm{eV}, 2.48 \pm 0.01 \mathrm{eV}$, and $3.04 \pm$ $0.01 \mathrm{eV}$ [27]. The CP energy of $1.55 \pm 0.01 \mathrm{eV}$ corresponds to $E_{\mathrm{g}}$ of $\alpha-\mathrm{FAPbI}_{3}$. Thus, $E_{\mathrm{g}}$ of $\alpha$-FAPbI $\mathrm{I}_{3}$ changes only slightly, if compared with $\mathrm{MAPbI}_{3}$.

On the other hand, $E_{\mathrm{g}}$ can be analyzed more conveniently using a $(\alpha E)^{2}-E$ plot. In Fig. 16.7(c), an example of this $E_{\mathrm{g}}$ analysis, performed for the $\alpha$ spectrum of $\mathrm{MAPbI}_{3}$, is shown [26]. In this analysis, the $E_{\mathrm{g}}$ value is determined from the intercept, but the analysis implemented using different $(\alpha E)^{2}$ regions gives different $E_{\mathrm{g}}$ values. Thus, the reliability of this analysis is rather low. Moreover, when the effect of surface roughness is underestimated in SE analyses, $E_{\mathrm{g}}$ is also underestimated (see Fig. 1.3). Accordingly, slightly smaller $E_{\mathrm{g}}$ values reported for $\mathrm{MAPbI}_{3}(1.50-1.61 \mathrm{eV}$ in Refs. $[10,12,16,29,54,67])$ and $\alpha-\mathrm{FAPbI}_{3}(1.43-1.53 \mathrm{eV}$ in Refs. $[4,15-17,21,29,68])$ could be interpreted by (i) the uncertainty in the $E_{\mathrm{g}}$ analysis and (ii) the contribution of surface roughness.

In Fig. 16.7(d), from the $\alpha$ spectrum of $\mathrm{MAPbI}_{3}$ and $\alpha-\mathrm{FAPbI}_{3}$, the Urbach energy $\left(E_{\mathrm{U}}\right)$ is further determined assuming $\alpha \propto \exp \left(E / E_{\mathrm{U}}\right)$ [i.e., $\ln (\alpha) \propto E / E_{\mathrm{U}}$ ]. The $E_{\mathrm{U}}$ values 
(a)

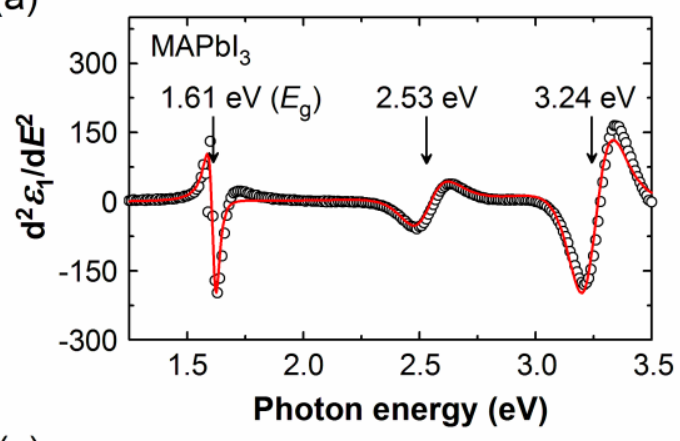

(c)

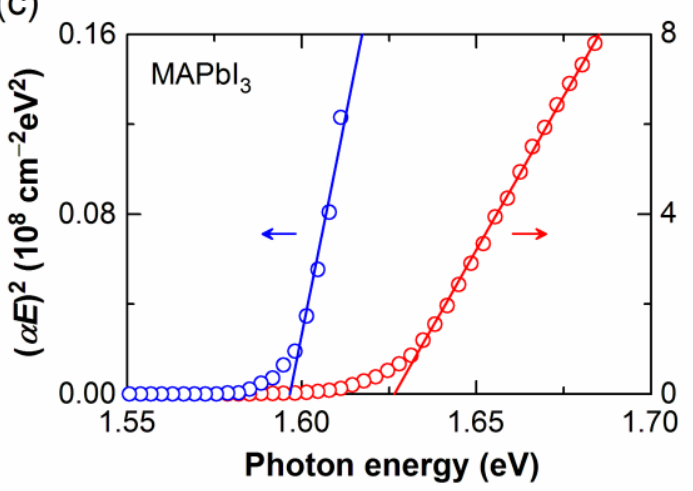

(b)

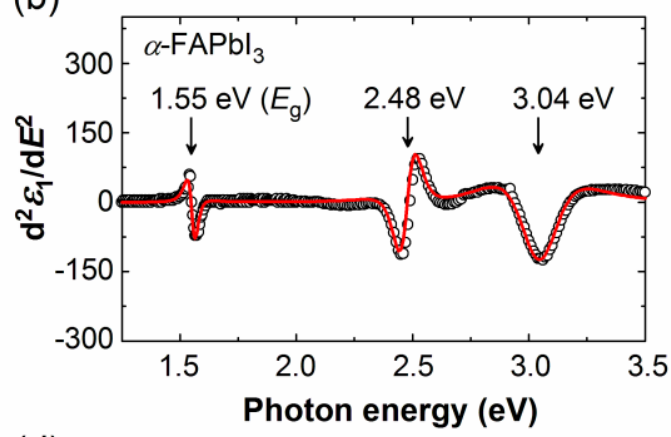

(d)

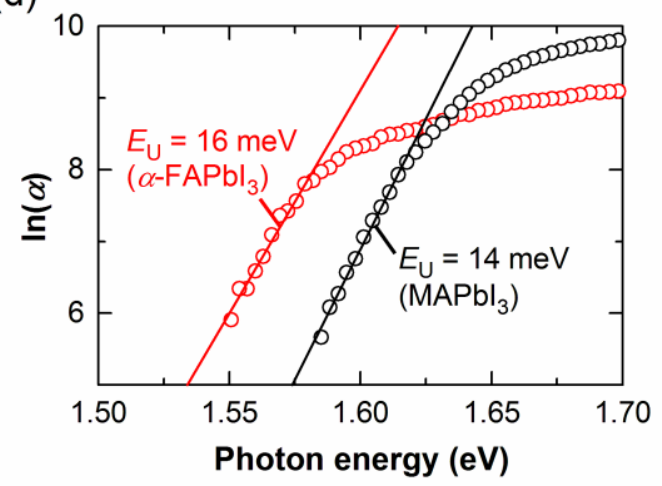

Fig. 16.7 Critical point (CP) analyses of (a) $\mathrm{MAPbI}_{3}$ [26] and (b) $\alpha-\mathrm{FAPbI}_{3}$ [27] dielectric functions, together with (c) $E_{\mathrm{g}}$ analysis for $\mathrm{MAPbI}_{3}$ using the $(\alpha E)^{2}-E$ plot [26] and (d) analysis of the Urbach energy $\left(E_{\mathrm{U}}\right)$ assuming $\ln (\alpha) \propto E / E_{\mathrm{U}}$. In the CP analyses of (a) and (b), the open circles denote the experimental data and the solid lines represent the theoretical fittings. From the $\mathrm{CP}$ analyses, $E_{\mathrm{g}}$ values are determined to be $1.61 \pm 0.01 \mathrm{eV}\left(\mathrm{MAPbI}_{3}\right)$ and $1.55 \pm 0.01 \mathrm{eV}\left(\alpha-\mathrm{FAPbI}_{3}\right)$. These $E_{\mathrm{g}}$ values correspond to the transition energies of $E_{0}$. In (c), the $E_{\mathrm{g}}$ analysis results for two different $(\alpha E)^{2}$ regions are shown, which result in different $E_{\mathrm{g}}$ values. The open circles show the experimental data and the solid lines represent the linear fitting results.

of $\mathrm{MAPbI}_{3}$ and $\alpha$-FAPbI ${ }_{3}$ estimated from this simple analysis are $14 \mathrm{meV}$ [26] and 16 $\mathrm{meV}$ [27], respectively, confirming the sharp absorption onset near $E_{\mathrm{g}}$ in hybrid perovskites [45].

In Fig. 16.8, the crystal structures and $\alpha$ spectra of $\delta$-FAPbI $3, \mathrm{MAPbI}_{3} \cdot \mathrm{H}_{2} \mathrm{O}$ and orthorhombic $\mathrm{CsPbI}_{3}\left(\delta-\mathrm{CsPb}_{3}\right)$ are shown. The crystal structure of $\delta$ - $\mathrm{FAPbI}_{3}$ in Fig. 16.8(a) is derived from the DFT calculation, while the reported crystal structures of $\mathrm{MAPbI}_{3} \cdot \mathrm{H}_{2} \mathrm{O}$ [69] and $\delta-\mathrm{CsPbI}_{3}[29]$ are shown in Figs. 16.8(b) and (c), respectively. 
(a) $\delta$ - $\mathrm{FPbl}_{3}$

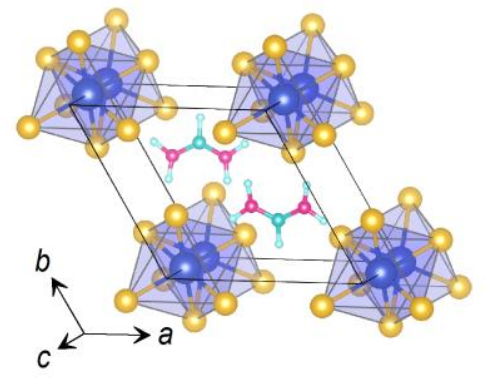

(b) $\mathrm{MAPbl}_{3} \cdot \mathrm{H}_{2} \mathrm{O}$

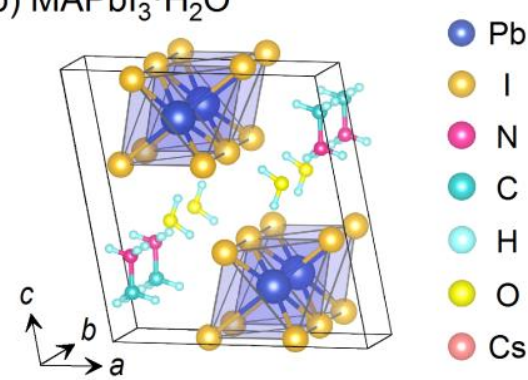

(c) $\delta$-CsPbl

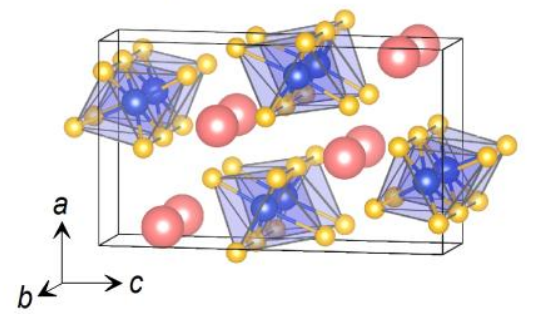

(d)

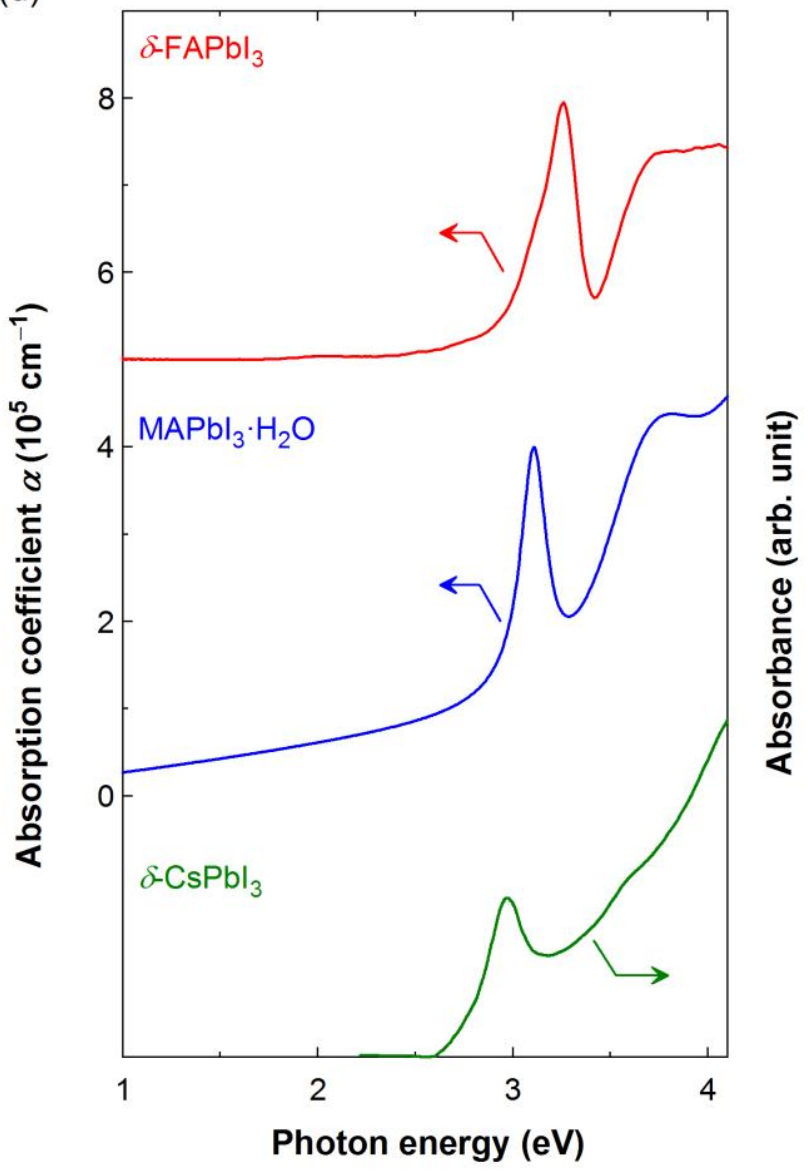

Fig. 16.8 Crystal structures of (a) $\delta$-FAPbI ${ }_{3}$, (b) $\mathrm{MAPbI}_{3} \cdot \mathrm{H}_{2} \mathrm{O}$ and (c) $\delta-\mathrm{CsPbI}_{3}$ and (d) their $\alpha$ spectra. The DFT-derived crystal structure $\left(\delta-\mathrm{FAPbI}_{3}\right)$ and reported crystal structures of $\mathrm{MAPbI}_{3} \cdot \mathrm{H}_{2} \mathrm{O}$ [69] and $\delta$ - $\mathrm{CsPbI}_{3}$ [29] are shown. All the crystals have similar one-dimensional crystal structures. In this figure, the $\alpha$ spectra reported for $\delta$-FAPbI ${ }_{3}$ [27], $\mathrm{MAPbI}_{3} \cdot \mathrm{H}_{2} \mathrm{O}$ [51] and $\delta-\mathrm{CsPbI}_{3}$ [70] are summarized. The $\alpha$ spectrum of $\delta$-FAPbI 3 is shifted by $5 \times 10^{5} \mathrm{~cm}^{-1}$ for clarity.

All the crystal phases have one-dimensional crystal structures consisting of $\mathrm{PbI}_{6}$ octahedra, although the crystal structure of $\delta$-FAPbI 3 is slightly different from the other two. It should be noted that, at room temperature, $\delta$ - $\mathrm{FAPbI}_{3}$ and $\delta-\mathrm{CsPbI}_{3}$ are the most stable phases of $\mathrm{FAPbI}_{3}$ and $\mathrm{CsPbI}_{3}$ [22]. On the other hand, it has been confirmed that the $\mathrm{MAPbI}_{3} \cdot \mathrm{H}_{2} \mathrm{O}$ phase is formed by the exposure of $\mathrm{MAPbI}_{3}$ to humid air [51] (Section 16.5). 
It can be seen from Fig. 16.8(d) that the reported $\alpha$ spectra of $\mathrm{MAPbI}_{3} \cdot \mathrm{H}_{2} \mathrm{O}$ [51] and $\delta-\mathrm{CsPbI}_{3}[70]$ are quite similar to that of $\delta$-FAPbI $\mathrm{F}_{3}$. In fact, all these materials show similar absorption peaks at $E=3.0-3.3 \mathrm{eV}$ with weak shoulder peaks at 3.6-3.8 eV. The sharp peak at $3.1 \mathrm{eV}$ in $\mathrm{MAPbI}_{3} \cdot \mathrm{H}_{2} \mathrm{O}$ has been attributed to the excitonic transition [51]. The DFT calculations confirmed that the interaction between the neighboring $\mathrm{PbI}_{6}$ octahedrons contributes to reduce $E_{g}$ and, as a result, $E_{\mathrm{g}}$ of $\mathrm{MAPbI}_{3}$ with three-dimensional $\mathrm{PbI}_{6}$ structure is much smaller, compared with two-dimensional $\mathrm{PbI}_{2}$ and one-dimensional $\mathrm{MAPbI}_{3} \cdot \mathrm{H}_{2} \mathrm{O}$ crystals [59].

\subsubsection{Optical Transitions in Hybrid Perovskites}

To understand the light absorption in hybrid perovskite crystals, the optical transition analyses have been implemented using DFT. In particular, the dielectric functions of $\mathrm{MAPbI}_{3}, \mathrm{MAPbBr}_{3}$ and $\alpha-\mathrm{FAPbI}_{3}$ can be reproduced quite well assuming simple pseudocubic structures based on DFT within PBE [26,27]. Moreover, universal rules that allow the unified interpretation of the light absorption in $\mathrm{APbX}_{3}$-type perovskites have been established [27]. In this section, these results obtained from the DFT analyses are introduced.

Figure 16.9 summarizes the DFT-derived crystal structures of (a) $\mathrm{MAPbI}_{3}$ [26], (b) $\alpha-\mathrm{FAPbI}_{3}$ [27] and (c) $\mathrm{MAPbBr}_{3}$ [27], and corresponding DFT dielectric functions of (d) $\mathrm{MAPbI}_{3}$ [26], (e) $\alpha-\mathrm{FAPbI}_{3}$ [27] and (f) $\mathrm{MAPbBr}_{3}$ [27]. The crystal structures of $\mathrm{MAPbI}_{3}$ and $\alpha-\mathrm{FAPbI}_{3}$ in Fig. 16.9 are identical to those shown in Fig. 16.1. The arrows indicate the $a, b$, and $c$ axes of the unit cells. In the optimized structures, the $\mathrm{C}-\mathrm{N}$ bonds of $\mathrm{MA}^{+}$in $\mathrm{MAPbI}_{3}$ and $\mathrm{MAPbBr}_{3}$ are aligned to be almost parallel to the $a$ axis, whereas the N-C-N plane of $\mathrm{FA}^{+}$in $\alpha-\mathrm{FAPbI}_{3}$ is parallel to the $a-b$ plane (or the line connecting two $\mathrm{N}$ atoms of $\mathrm{FA}^{+}$is parallel to the $a$ axis). The orientation of $\mathrm{FA}^{+}$in $\alpha$-FAPbI 3 [Fig. 16.9(b)] is consistent with the experimental result [19].

In Figs. 16.9(d)-(f), the experimental dielectric functions shown in Fig. 16.5 (open circles) are compared with the DFT-derived dielectric functions (solid lines). The theoretical dielectric functions have been obtained assuming the different polarization states with directions parallel to the $a, b$ and $c$ axes in Figs. 16.9(a)-(c), and the arrows indicated in Figs. 16.9(d)-(f) represent the transition (CP) energies in the Brillouin zone described below. For $\mathrm{MAPbBr}_{3}$, however, all the calculated spectra have been shifted toward higher energy by $0.22 \mathrm{eV}$ to match the DFT result with the experimental result. The DFT calculations show that all the perovskite compounds have highly anisotropic optical characteristics. In the case of $\mathrm{MAPbI}_{3}$, for example, the dielectric function for 
(a) $\mathrm{MAPbl}_{3}$

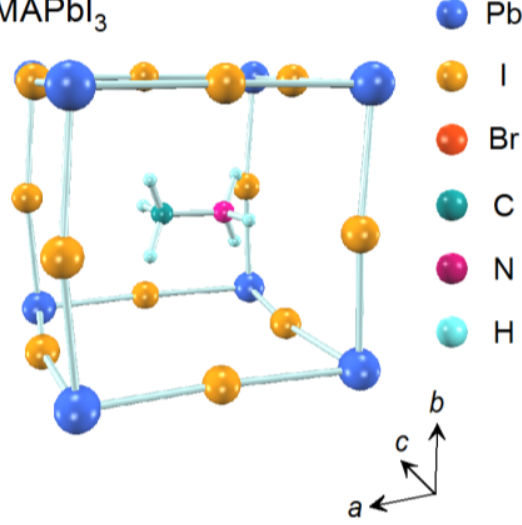

(b) $\alpha-\mathrm{FAPbl}_{3}$

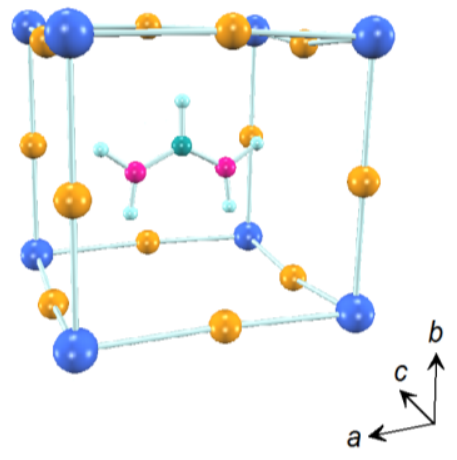

(c) $\mathrm{MAPbBr}_{3}$

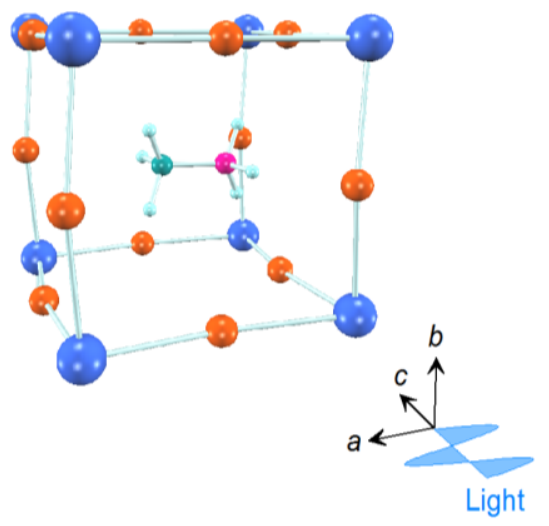

(d)

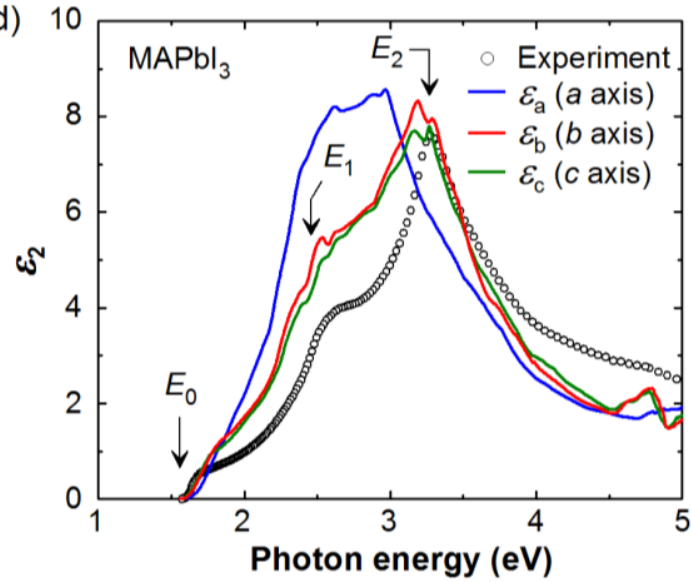

(e)

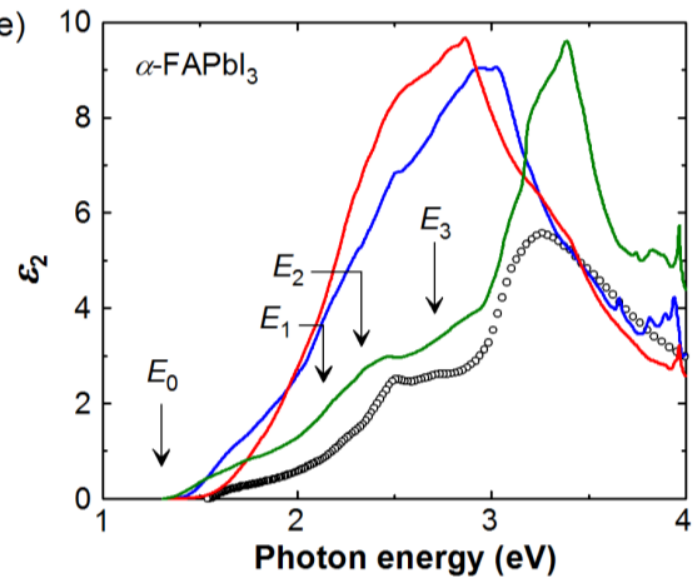

(f)

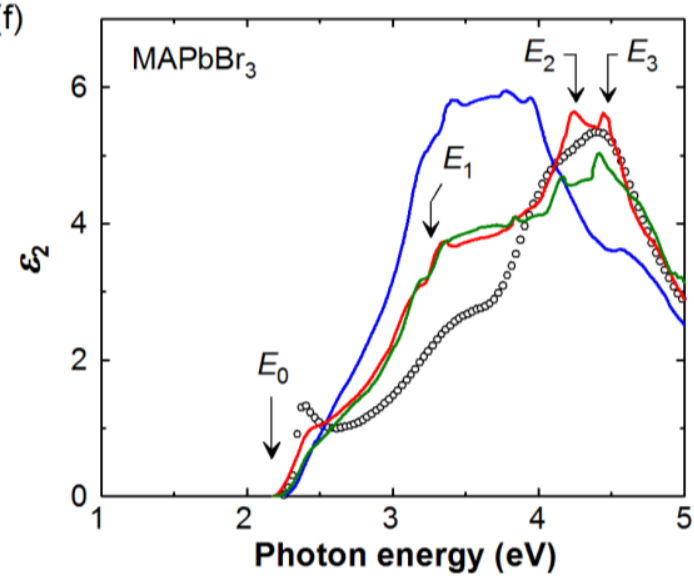

Fig. 16.9 Pseudocubic crystal structures of (a) $\mathrm{MAPbI}_{3}$, (b) $\alpha-\mathrm{FAPbI}_{3}$ and (c) $\mathrm{MAPbBr}_{3}$ obtained from the DFT calculations, and $\varepsilon_{2}$ spectra of (d) $\mathrm{MAPbI}_{3}$, (e) $\alpha-\mathrm{FAPbI}_{3}$ and (f) $\mathrm{MAPbBr}_{3}$, obtained from the experiments (open circles) and DFT calculations (solid lines). In (a)-(c), the arrows indicate the $a, b$, and $c$ axes of the unit cells. In (d)-(f), the experimental results correspond to those shown in Fig. 16.5, and $\varepsilon_{\mathrm{a}}, \varepsilon_{\mathrm{b}}$ and $\varepsilon_{\mathrm{c}}$ represent the $\varepsilon_{2}$ spectra calculated assuming the light polarization along the $a, b$, and $c$ axes in (a)-(c), respectively. On the $\varepsilon_{2}$ spectra, the transition energies determined by the DFT analyses (see Fig. 16.10) are indicated. In (f), the DFT spectra of $\mathrm{MAPbBr}_{3}$ have been shifted toward higher energy by $0.22 \mathrm{eV}$ to obtain better matching with the experimental spectrum. The experimental and DFT data are taken from Ref. [26] (MAPbI $\left.{ }_{3}\right)$ and Ref. [27] $\left(\alpha-\mathrm{FAPbI}_{3}\right.$ and $\left.\mathrm{MAPbBr}_{3}\right)$. 
the $a$ axis polarization $\left(\varepsilon_{\mathrm{a}}\right)$ is quite different to those for the $b$ and $c$ axes ( $\varepsilon_{\mathrm{b}}$ and $\varepsilon_{\mathrm{c}}$ ), and similar trends can be confirmed for $\mathrm{MAPbBr}_{3}$. It can be seen that the overall shapes of $\varepsilon_{\mathrm{b}}$ and $\varepsilon_{\mathrm{c}}$ obtained for $\mathrm{MAPbI}_{3}$ and $\mathrm{MAPbBr}_{3}$ show remarkable agreement with those of the experimental spectra. In the case of $\alpha-\mathrm{FAPbI}_{3}$, the $\varepsilon_{2}$ spectra for the $a$ and $b$ axes $\left(\varepsilon_{\mathrm{a}}\right.$ and $\varepsilon_{\mathrm{b}}$ ) exhibit different shapes from that for the $c$ axis $\left(\varepsilon_{\mathrm{c}}\right)$, and the shape of $\varepsilon_{\mathrm{c}}$ agrees well with the experimental dielectric function.

In Figs. 16.9(d)-(f), the CP energies $\left(E_{0-3}\right)$ of $\mathrm{MAPbI}_{3}, \mathrm{MAPbBr}_{3}$ and $\alpha-\mathrm{FAPbI}_{3}$, deduced from the DFT calculations, show excellent agreement with the experimental results, and all the fine absorption features observed experimentally in these perovskites are reproduced quite well. However, the band-edge excitonic transition observed in $\mathrm{MAPbBr}_{3}$ at $2.4 \mathrm{eV}$ is not reproduced in the calculation result as the DFT calculations in Fig. 16.9 have been performed without incorporating electron-hole interactions (or exciton formation). Consequently, the calculated $\varepsilon_{2}$ becomes lower than the experimental $\varepsilon_{2}$ near the $E_{\mathrm{g}}$ region. In contrast, excellent matching is observed between the experimental and DFT spectra for $\mathrm{MAPbI}_{3}$ and $\alpha-\mathrm{FAPbI}_{3}$, confirming that the contribution of excitons in the light absorption process is negligible in these materials $[26,27]$. Non-excitonic nature of $\mathrm{MAPbI}_{3}$ has already been confirmed and discussed quite extensively $[47,71,72]$.

Figure 16.10 shows the band structure and the density of states (DOS) of (a) $\mathrm{MAPbI}_{3}$ [26] and (b) $\alpha-\mathrm{FAPbI}_{3}$ [27], calculated from the pseudocubic structures of Figs. 16.9(a) and (b), respectively. For the band structures, the corresponding Brillouin zone is shown in the inset and the partial DOS of each material is also shown. The $V_{j}$ and $C_{j}$ in these figures denote the $j$ th valence and conduction bands from the valence band maximum (VBM) and the conduction band minimum (CBM), respectively. In both $\mathrm{MAPbI}_{3}$ and $\alpha-\mathrm{FAPbI}_{3}, V_{1}$ consists of the $\mathrm{Pb} 6 s$ and I $5 p$ states, whereas $C_{1}$ is dominated by $\mathrm{Pb} 6 p$ $[9,26,64,73,74]$, as confirmed from the corresponding partial DOS. The band structures of $\mathrm{MAPbI}_{3}$ and $\alpha-\mathrm{FAPbI}_{3}$ are quite similar, but $\alpha-\mathrm{FAPbI}_{3}$ has an additional conduction band at $\sim 2.9 \mathrm{eV}$ due to the $\pi$-state of the $s p^{2} \mathrm{C}$ atom in $\mathrm{FA}^{+}$. The insets of Fig. 16.10(a) also show the charge density profiles of $\mathrm{MAPbI}_{3}$ for the designated energy regions of $E$ $=1.4 \sim 1.6 \mathrm{eV}(\mathrm{CBM}), E=-0.2 \sim 0.0 \mathrm{eV}(\mathrm{VBM})$, and $E=-0.6 \sim-0.4 \mathrm{eV}$. Because of the antibonding nature of $\mathrm{Pb}-\mathrm{I}$ [75], the charge densities near VBM are localized on the $\mathrm{Pb}(6 s)$ and $\mathrm{I}(5 p)$, while the charge density near $\mathrm{CBM}$ is dominated by $\mathrm{Pb} 6 p$. In the band structures of Fig. 16.10, the energy positions of $M_{1-3}$ and those of $X_{1-3}$ differ slightly because the assumed cubic crystal is distorted and the resulting reciprocal lattices $\left(a^{*}=2 \pi / a, b^{*}=2 \pi l b, c^{*}=2 \pi / c\right)$ are different. In particular, the $\alpha$-FAPbI 3 unit cell is distorted largely due to the steric effect of $\mathrm{FA}^{+}$and the energy differences of $M_{1-3}$ 

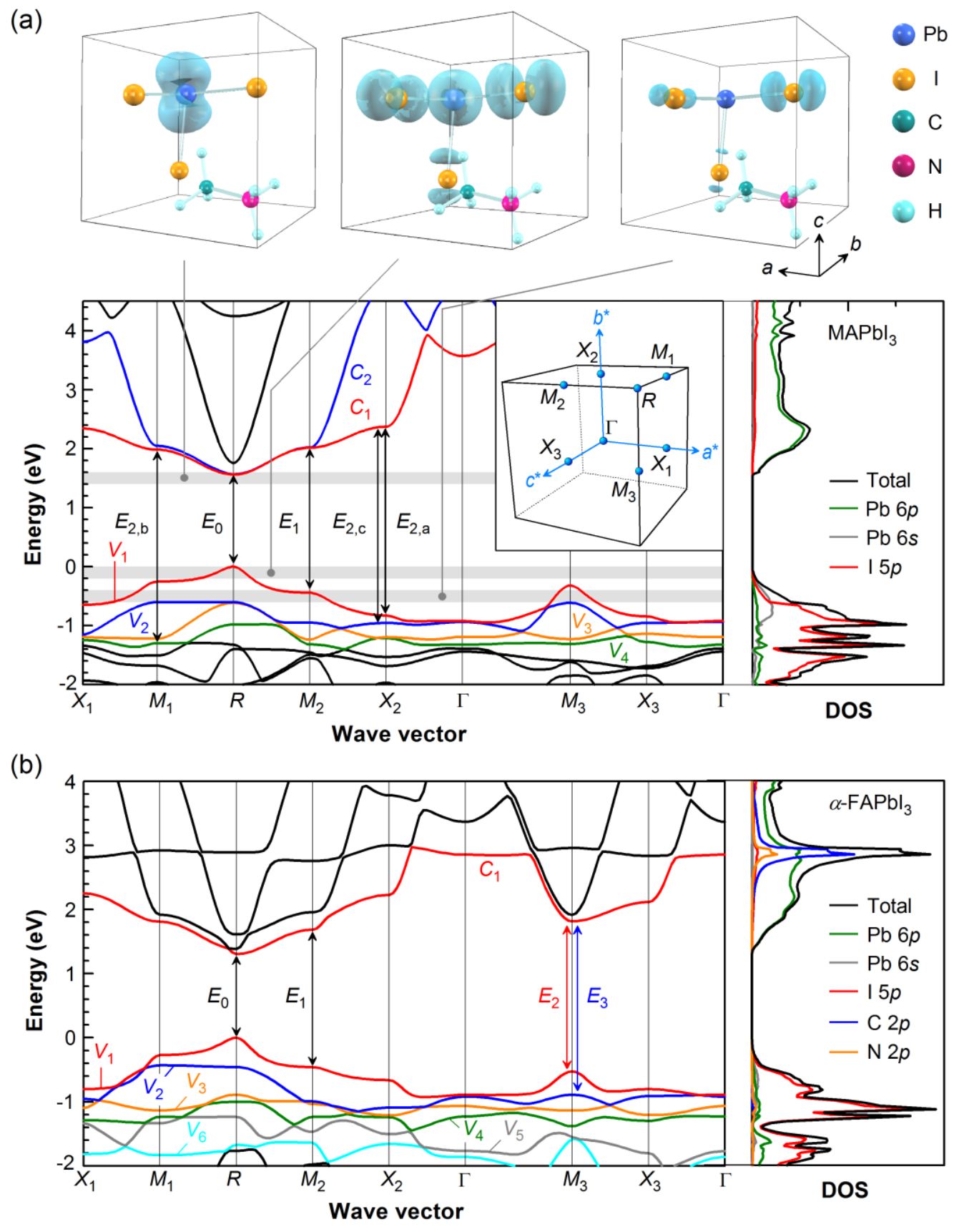

Fig. 16.10 Band structure and DOS of (a) $\mathrm{MAPbI}_{3}$ [26] and (b) $\alpha-\mathrm{FAPbI}_{3}$ [27] pseudocubic crystals. In (a) and (b), $V_{j}$ and $C_{j}$ denote the $j$ th valence and conduction bands from VBM and CBM, respectively, and the partial DOS distributions are also indicated. The optical transitions determined by the polarization-dependent DFT analyses are indicated by arrows and the energy positions of these transitions are shown in Fig. 16.9. The inset of (a) shows the high symmetry points in the Brillouin zone defined by the reciprocal lattices $\left(a^{*}=2 \pi / a, b^{*}=2 \pi / b, c^{*}=2 \pi / c\right)$ of the assumed pseudocubic structure and the charge density profiles for the designated energy regions of $E=-0.2 \sim 0.0 \mathrm{eV}(\mathrm{VBM}), E=1.4 \sim 1.6 \mathrm{eV}(\mathrm{CBM})$ and $E=-0.6 \sim-0.4 \mathrm{eV}$. 
(a)

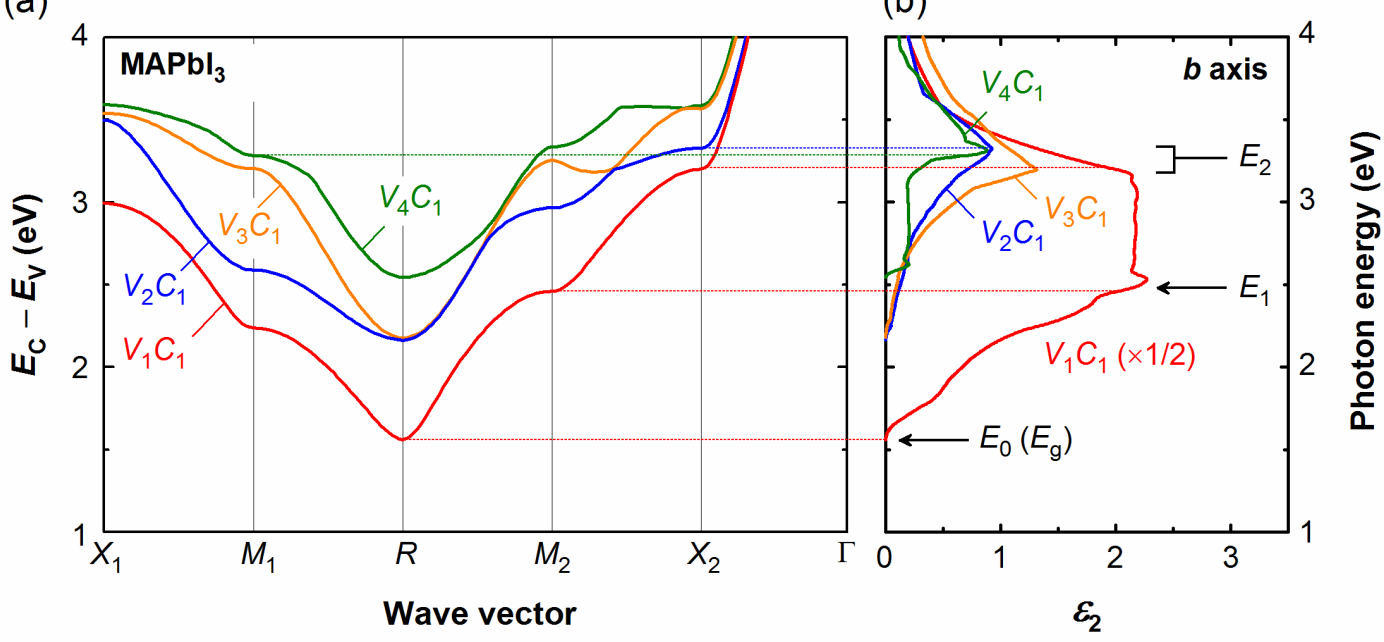

Fig. 16.11 (a) Energy separation between the conduction and valence bands $\left(E_{\mathrm{C}}-E_{\mathrm{V}}\right)$ in $\mathrm{MAPbI}_{3}$ and (b) contributions of various interband transitions to the $\varepsilon_{2}$ spectrum of $\mathrm{MAPbI}_{3}$. In (a), $V_{j} C_{1}(j \leq 4)$ denote the interband transitions of $V_{j} \rightarrow C_{1}$ in Fig. 16.10(a). In (b), the $\varepsilon_{2}$ contributions of $V_{j} C_{1}$ for the $b$ axis (i.e., $\varepsilon_{\mathrm{b}}$ ) are shown, and the amplitude of the $\varepsilon_{2}$ spectrum for the $V_{1} C_{1}$ transition is reduced to half for clarity. The data reported in Ref. [77] are shown.

and $X_{1-3}$ are larger in $\alpha-\mathrm{FAPbI}_{3}$ than in $\mathrm{MAPbI}_{3}$.

The arrows in Figs. 16.10(a) and (b) represent the interband transitions that occur at high symmetry points in the pseudocubic Brillouin zone. These optical transitions have been characterized by calculating the dielectric response of each interband transition by DFT [26,27], as described below. The energy positions of the arrows in Figs. 16.9(d)-(f) correspond to the interband transition energies obtained from the corresponding band structures. In the case of $\mathrm{MAPbI}_{3}$, however, the $E_{2}$ transition consists of three components and each component is labeled using the subscript of $a, b$ or $c$. It can be seen that $\mathrm{MAPbI}_{3}[9,26,64]$ and $\alpha-\mathrm{FAPbI}_{3}[27,76]$ are direct transition semiconductors with the $E_{0}\left(E_{\mathrm{g}}\right)$ transition at the $R$ point (cubic symmetry) and other hybrid perovskites also show direct gaps $[27,76]$. The transition energies of $E_{0-3}$ obtained from the band structures show remarkable agreement with the experimental values, as confirmed from Fig. 16.9(d)-(f).

Figure 16.11 shows the optical transition analysis performed for $\mathrm{MAPbI}_{3}$ [77]. In this analysis, the energy separation between the conduction and valence bands $\left(E_{\mathrm{C}}-E_{\mathrm{V}}\right)$ is calculated first for each interband transition of $V_{j} \rightarrow C_{k}$. In Fig. 16.11(a), the calculated 
results for $V_{j} C_{1}(j \leq 4)$ of Fig. 16.10(a) are shown, where $V_{j} C_{1}$ denote the transition of $V_{j}$ $\rightarrow C_{1}$. The $\varepsilon_{2}$ contributions induced by the $V_{j} C_{1}$ transitions are calculated further [Fig. 16.11(b)] and are compared with the energy separations in Fig. 16.11(a). From this procedure, the optical transitions at high symmetry points that satisfy van Hove singularities [78] in $\mathbf{k}$ space (i.e., $\nabla_{\mathbf{k}}\left[E_{\mathrm{C}}(\mathbf{k})-E_{\mathrm{V}}(\mathbf{k})\right]=0$ ) have been determined. It can be seen from Fig. 16.11(b) that the optical transition in the visible region is dominated by the $V_{1} C_{1}$ transition. In Fig. 16.11(b), the $\varepsilon_{2}$ contributions for the $b$-axis component [i.e., $\varepsilon_{b}$ in Fig. 16.9(d)] are shown and, in this case, only the transitions at the $R, M_{1}, M_{2}$, and $X_{2}$ points are allowed (i.e., $b^{*}$ component of the Brillouin zone). From these assumptions, the $\mathrm{CP}$ energies observed in the $\varepsilon_{2}$ spectrum of $\mathrm{MAPbI}_{3}$ are assigned to the interband transitions of $V_{1} C_{1}$ at the $R$ ( $E_{0}$ transition), $M_{2}\left(E_{1}\right.$ transition) and $X_{2}\left(E_{2, \mathrm{a}}\right.$ transition) points. Moreover, the $\varepsilon_{2}$ peaks for the $V_{2} C_{1}$ and $V_{4} C_{1}$ transitions are attributed to the transitions of $V_{4} C_{1}$ at the $M_{1}$ point ( $E_{2, \mathrm{~b}}$ transition) and $V_{2} C_{1}$ at the $X_{2}$ point $\left(E_{2, \mathrm{c}}\right.$ transition). The CP energies observed in Fig. 16.11(a), however, deviate slightly from the exact peak positions confirmed in the calculated $\varepsilon_{2}$ spectra and thus slight ambiguity remains. Similar optical transition analyses have been made for $\alpha-\mathrm{FAPbI}_{3}$ and $\mathrm{MAPbBr}_{3}$ [27].

The above DFT analysis and partial DOS in Fig. 16.10 indicate that direct semiconductor-type transitions occur within the $\mathrm{PbI}_{3}{ }^{-}$component in $\mathrm{MAPbI}_{3}$ and $\alpha$-FAPbI ${ }_{3}$ in the visible region $(E<4 \mathrm{eV})$, supporting the efficient carrier generation in these semiconductors [26,27,77]. By applying the self-consistent $G W(\operatorname{sc} G W)$ approximation, the optical transition analyses of $\mathrm{MAPbX}_{3}$ have been performed and slightly different assignments have been proposed for $\mathrm{MAPbI}_{3}$ [52]. It should be emphasized that the DFT-derived dielectric function changes rather significantly depending on calculation (approximation) method. As examples, Fig. 16.12 compares the $\alpha$ spectra of (a) $\mathrm{MAPbI}_{3}$ and (b) $\alpha-\mathrm{FAPbI}_{3}$ calculated by DFT using different approximations. In these figures, the $\alpha$ values in linear and logarithmic scales are summarized. For $\mathrm{MAPbI}_{3}$, the DFT calculation results obtained within PBE [26], the $G W$ approximation [30], and the $\operatorname{sc} G W$ approximation [52] are shown. In the $G W$ and sc $G W$ calculations, the effect of the spin-orbit coupling (SOC) [79] has been taken into account. For the $G W$-SOC results [30], however, only relative $\alpha$ values have been reported, and the absolute $\alpha$ values are deduced by assuming that the $\alpha$ values at $3.0 \mathrm{eV}$ are identical to those of the PBE results. All the DFT calculations reproduce $E_{\mathrm{g}}$ of $\mathrm{MAPbI}_{3}$ successfully, as discussed previously [52,73,80-82]. In general, $E_{\mathrm{g}}$ is underestimated seriously in PBE [83]. For hybrid perovskites, however, the $E_{\mathrm{g}}$ values calculated by $\mathrm{PBE}$ agree quite well with the experimental values, which has been 
(a) $\mathrm{MAPbl}_{3}$

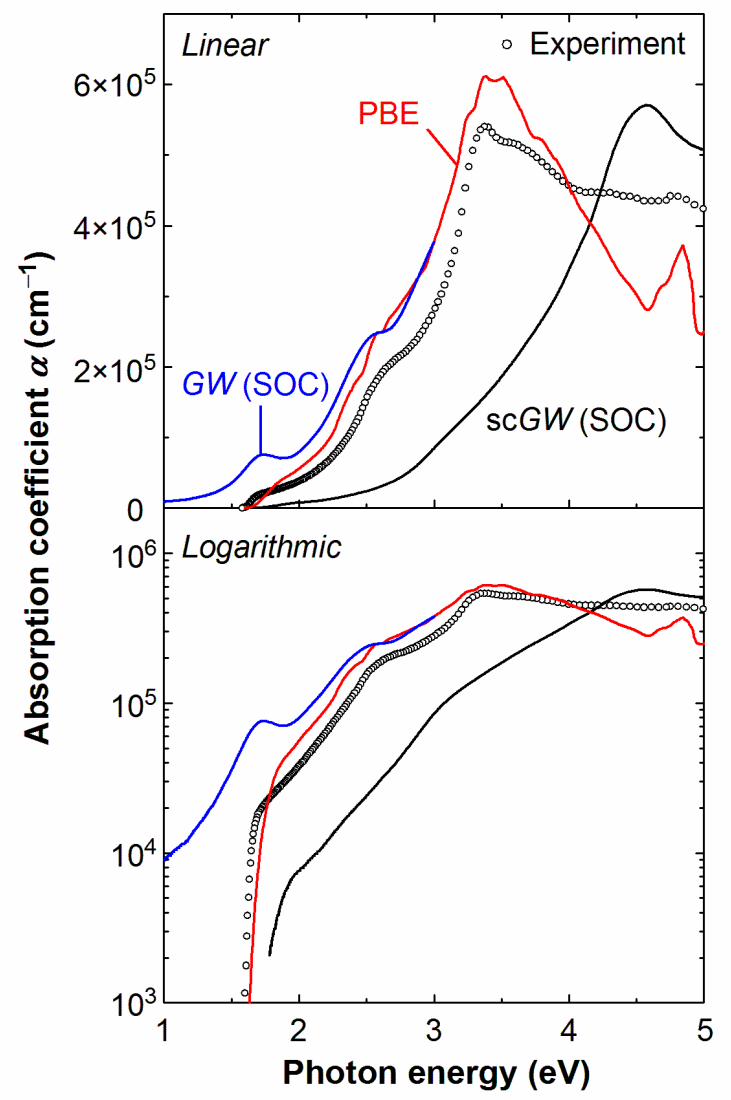

(b) $\alpha-\mathrm{FAPbl}_{3}$

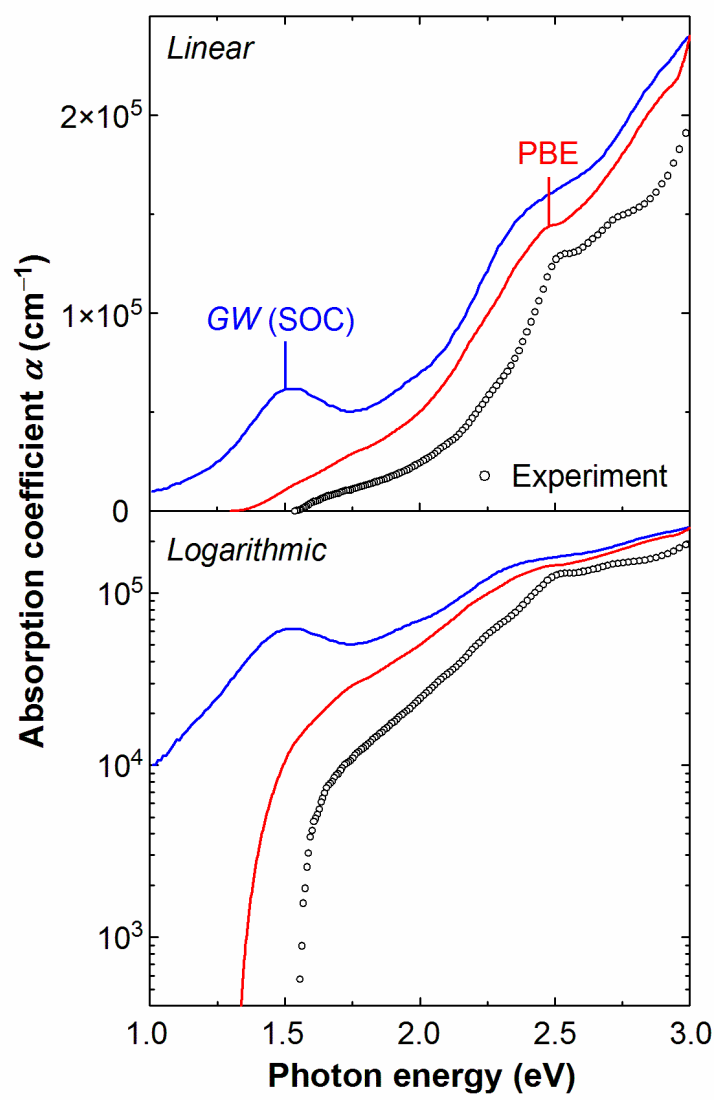

Fig. 16.12 $\alpha$ spectra of (a) $\mathrm{MAPbI}_{3}$ and (b) $\alpha$-FAPbI ${ }_{3}$ calculated by DFT using different approximations: $\mathrm{PBE}\left(\mathrm{MAPbI}_{3}\right.$ [26] and $\alpha-\mathrm{FAPbI}_{3}$ [27]), $G W$ approximation with $\mathrm{SOC}$ [30], and self-consistent $G W(\operatorname{sc} G W)$ approximation with SOC [52]. In (a) and (b), the $\alpha$ values in linear and logarithmic scales are shown. The absolute $\alpha$ values of the $G W$ (SOC) results [30] are deduced by assuming that the $\alpha$ values at $3.0 \mathrm{eV}$ are identical to those of the PBE results.

attributed to the cancellation of errors in the DFT calculations $[52,73,79]$. On the other hand, if the SOC interaction is not considered in the $G W$ and $\operatorname{sc} G W$, the $E_{\mathrm{g}}$ values are overestimated seriously [52,80-82].

The $\operatorname{sc} G W$ approximation is generally accepted as the most advanced DFT calculation method. However, the transition energies of the $\alpha$ spectrum obtained from the $\operatorname{sc} G W$-SOC occur at higher energies and the absolute $\alpha$ values are much smaller, compared with the experimental result. In the $G W$-SOC results of $\mathrm{MAPbI}_{3}$ and $\alpha$-FAPbI ${ }_{3}$, small peaks appear near $E_{\mathrm{g}}$ and the absorption features near the fundamental 
band edge are not reproduced well. As known widely [30,52,79-81], when the DFT calculations are implemented by incorporating SOC, all the bands split into two bands and the band structure becomes slightly indirect (or pseudo-direct). Thus, the $\alpha$ peak observed in the $G W$-SOC results could be attributed to the SOC effect. It has been confirmed that the SOC-induced band splitting near the fundamental gap is quite sensitive to the orientation and position of the A-site cation [84-86].

In contrast, the absolute $\alpha$ values and peak energies, deduced from the PBE calculations, show excellent agreement with the experimental spectra, although $E_{\mathrm{g}}$ of $\alpha-\mathrm{FAPbI}_{3}$ is underestimated. At least at this stage, therefore, the PBE calculations provide the best matching with the experimental data. Moreover, the PBE results reproduce the experimental tail-state absorption quite well with similar Urbach energies $\left(E_{\mathrm{U}} \sim 15 \mathrm{meV}\right)$. This is rather surprising as the DFT spectra are obtained assuming a perfect crystal structure with no phonon interaction $(0 \mathrm{~K})$. Accordingly, the room-temperature optical spectra of the hybrid perovskites, obtained from the experiments, exhibit ideal sharp absorption feature with quite suppressed tail-state absorption.

\subsubsection{Universal Rules for Light Absorption in $\mathrm{APbX}_{3}$ Perovskites}

The large $\alpha$ reduction induced by the replacement of $\mathrm{MA}^{+}$with $\mathrm{FA}^{+}$in $\mathrm{APbI}_{3}$ (Fig. 16.5) and the strong optical anisotropy confirmed in the DFT calculations (Fig. 16.9) are found to originate from the strong interaction of the A-site cation with the X-site halogen atom [27]. In particular, the visible light absorption in $\mathrm{APbX}_{3}$ varies rather significantly depending on the A-site cation species. Here, we discuss the optical absorption phenomena in hybrid perovskites based on more detailed DFT analyses using PBE [27].

Figure 16.13 represents the charge density profiles of (a) $\alpha-\mathrm{FAPbI}_{3}$, (b) $\mathrm{MAPbI}_{3}$ and (c) $\mathrm{CsPbI}_{3}$ in the energy region of $-0.6 \sim-0.4 \mathrm{eV}$ from $\mathrm{VBM}(E=0 \mathrm{eV})$. In this figure, the charge density indicated by the colors decreases with the order of red $>$ yellow $>$ green $>$ blue with red being the highest. These results have been obtained from the DFT-optimized structures (see Fig. 16.9 for $\mathrm{MAPbI}_{3}$ and $\alpha-\mathrm{FAPbI}_{3}$ ). The charge density of $\mathrm{MAPbI}_{3}$ has already been shown in the inset of Fig. 16.10(a).

The charge densities of the selected energy region $(-0.6 \sim-0.4 \mathrm{eV})$ correspond to the valence electron states that involve in the $E_{1}$ transition in $\mathrm{MAPbI}_{3}(2.46 \mathrm{eV})$ and the $E_{2}$ transition in $\alpha-\mathrm{FAPbI}_{3}(2.34 \mathrm{eV})$, as confirmed from the $V_{1}$ energy positions at the $M$ points in Fig. 16.10. Here, the valence charge states at the $M$ points are considered 
(a) $\alpha-\mathrm{FAPbl}_{3}$
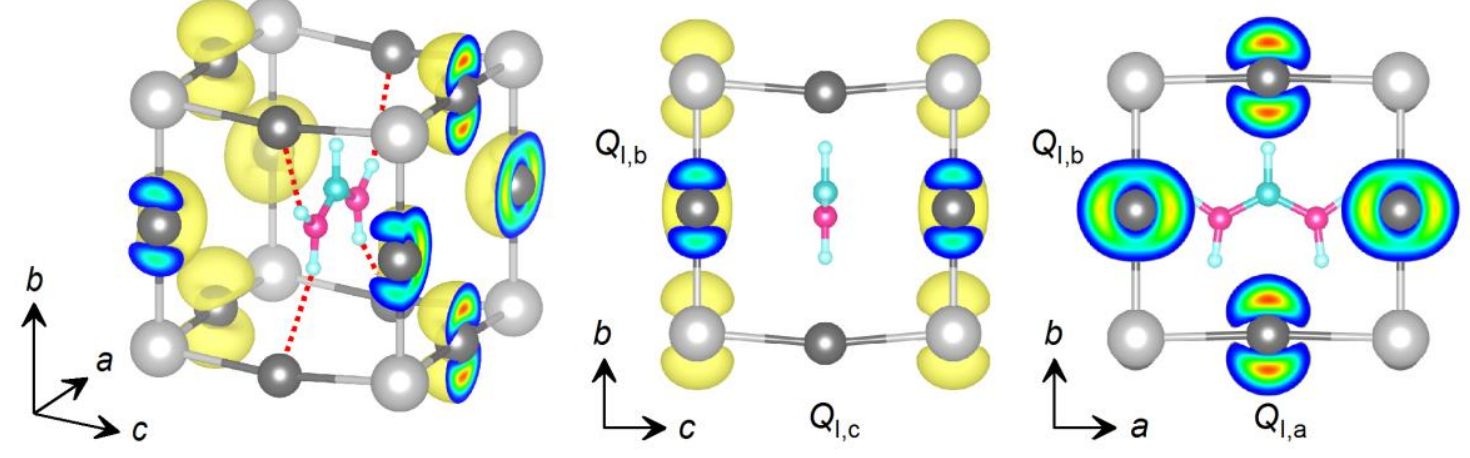

(b) $\mathrm{MAPbl}_{3}$
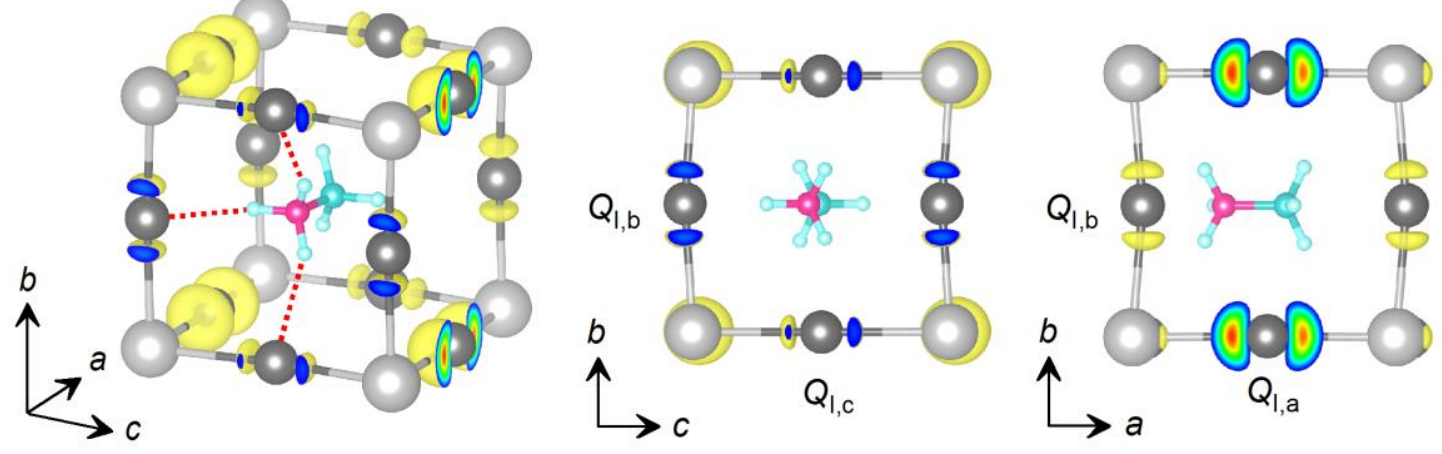

(c) $\mathrm{CsPbl}_{3}$
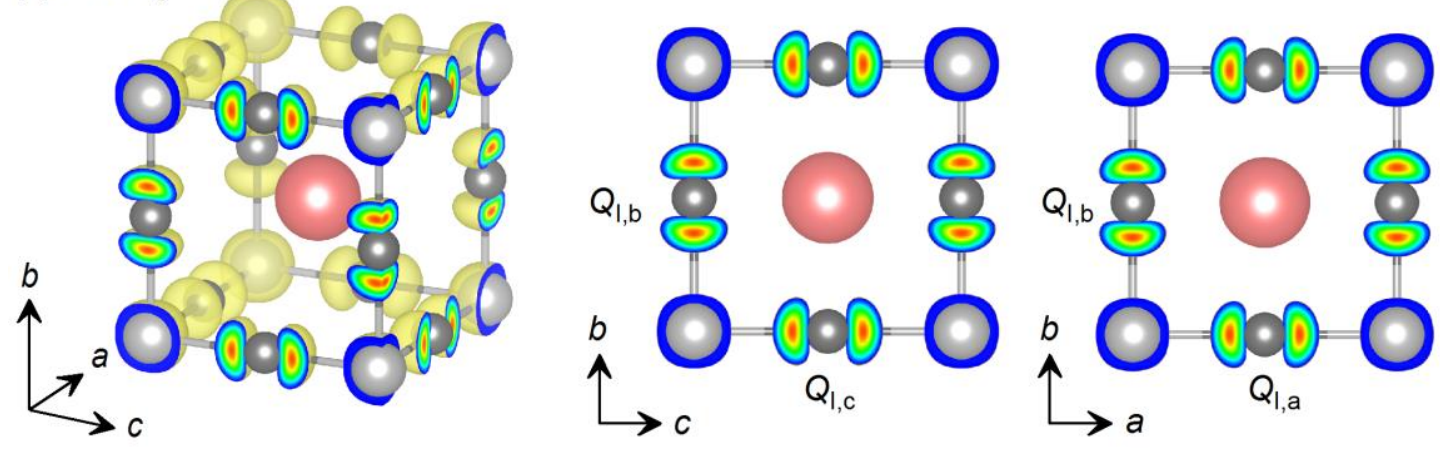

$\bigcirc \mathrm{Pb} \odot \mathrm{I} \odot \mathrm{C} \odot \mathrm{N} \odot \mathrm{H} \odot \mathrm{Cs}$

Fig. 16.13 Charge density profiles of (a) $\alpha-\mathrm{FAPbI}_{3}$, (b) $\mathrm{MAPbI}_{3}$ and (c) $\mathrm{CsPbI}_{3}$ in the energy region of $-0.6 \sim-0.4 \mathrm{eV}$ from $\mathrm{VBM}(E=0 \mathrm{eV})$. These profiles represent the valence charge densities for the $E_{2}$ transition $\left(\alpha-\mathrm{FAPbI}_{3}\right)$ and the $E_{1}$ transition $\left(\mathrm{MAPbI}_{3}\right.$ and $\left.\mathrm{CsPb}_{3}\right)$ at the $M$ point $\left(V_{1} C_{1}\right)$. The charge density decreases with the order of red $>$ yellow $>$ green $>$ blue with red being the highest. The arrows show the $a, b$, and $c$ axes of the unit cells, and the valence charges of the I atoms along the $a, b$, and $c$ axes are indicated as $Q_{\mathrm{I}, \mathrm{a}}, Q_{\mathrm{I}, \mathrm{b}}$ and $Q_{\mathrm{I}, \mathrm{c}}$, respectively. For $\alpha-\mathrm{FAPbI}_{3}$ and $\mathrm{MAPbI}_{3}$, the data reported in Ref. [27] are shown. 
specifically, as the visible light absorption at $\sim 2 \mathrm{eV}$ in the hybrid perovskites is characterized by the interband transitions near the $M$ point $\left(V_{1} C_{1}\right)$. Since the transition probability is determined by the interaction of incoming polarized light with the valence state [see Eq. (4.2)], the optical absorption can be studied more directly based on the valence electron (wave function) in the corresponding energy range. Although the conduction states are also important in determining the transition probability, the effect of the $\mathrm{Pb}$-derived conduction bands is treated as a constant contribution in this analysis.

In Fig. 16.13, the charge density profiles of $\mathrm{APbI}_{3}$ in the same energy region (-0.6 $-0.4 \mathrm{eV}$ ) are shown, but the distribution of the I-5p charge densities varies significantly depending on the cation species. More specifically, the I-5p valence electron of $\alpha$-FAPbI ${ }_{3}$ concentrates on the I atoms along the $a$ and $b$ axes, and the valence charges of these I atoms $\left(Q_{\mathrm{I}, \mathrm{a}}\right.$ and $Q_{\mathrm{I}, \mathrm{b}}$, respectively) are far larger than that of the $c$ axis direction $\left(Q_{\mathrm{I}, \mathrm{c}}\right)$. In the case of $\mathrm{MAPbI}_{3}$, the valence charge distribution is more uniform, even though $Q_{\mathrm{I}, \mathrm{a}}$ is larger than $Q_{\mathrm{I}, \mathrm{b}}$ and $Q_{\mathrm{I}, \mathrm{c}}$, while quite homogeneous charge distribution $\left(Q_{\mathrm{I}, \mathrm{a}} \sim Q_{\mathrm{I}, \mathrm{b}} \sim Q_{\mathrm{I}, \mathrm{c}}\right)$ can be seen for $\mathrm{CsPbI}_{3}$.

It has been confirmed that the distribution of $Q_{\mathrm{I}, \mathrm{a}-\mathrm{c}}$ is modified strongly by the hydrogen bonding interaction expressed by $\mathrm{I}\left(\delta^{-}\right) \cdots \mathrm{H}\left(\delta^{+}\right)-\mathrm{N}\left(\delta^{-}\right)$[27], where $\delta^{+}$and $\delta^{-}$ denote the positive and negative partial charges, respectively, and the magnitude of $\delta$ is determined mainly by the electronegativity. In particular, the hydrogen bondings of $\mathrm{I} \cdots \mathrm{H}-\mathrm{N}$, indicated by the dotted lines in Fig. 16.13(a) and (b), lead to the reduction of the N-I distance $[26,33]$. In the case of $\mathrm{MAPbI}_{3}$, for example, the $\mathrm{N}$ atom position of $\mathrm{MA}^{+}$is located more closely to the $b-c$ plane due to the hydrogen bondings formed with the I atoms in the $b$ and $c$ axis directions. The result of Fig. 16.13 shows clearly that the distribution of $Q_{\mathrm{I}, \mathrm{a}-\mathrm{c}}$ is governed primarily by the N-I distance and $Q_{\mathrm{I} \text {,a-c }}$ decreases significantly when the N-I distance is smaller. This electrostatic interaction between the $\mathrm{N}$ and I atoms, referred to as the anti-coupling effect hereafter, plays a critical role for the interpretation of the visible light absorption in hybrid perovskites. In $\alpha-\mathrm{FAPbI}_{3}$, the intense anti-coupling effect leads to the significant reduction of $Q_{\mathrm{I}, \mathrm{c}}$ due to the short distance between the I ( $c$ axis) and $\mathrm{N}$ atoms. As a result, the valence charge of I $5 p$ concentrates on the $a-b$ plane. In $\mathrm{MAPbI}_{3}$, smaller $Q_{\mathrm{I}, \mathrm{b}}$ and $Q_{\mathrm{I}, \mathrm{c}}$, compared with $Q_{\mathrm{I}, \mathrm{a}}$, can also be interpreted by the strong anti-coupling interaction as the N-I distance is shorter for the $\mathrm{I}$ atoms on the $b$ and $c$ axes. In contrast, the anti-coupling interaction is negligible in $\mathrm{CsPbI}_{3}$ due to the lack of the hydrogen bonding.

The highly anisotropic optical transitions at the $M$ point, observed in the DFT results of Fig. 16.9, can be understood based on the above anti-coupling effect. In the case of $\alpha-\mathrm{FAPbI}_{3}$, for example, the $\varepsilon_{2}$ value at $2.34 \mathrm{eV}$ corresponds to the $E_{2}$ transition, and $\varepsilon_{\mathrm{b}}$ 
shows the highest $\varepsilon_{2}$ value at this energy, while $\varepsilon_{\mathcal{c}}$ shows the lowest value [Fig. 16.9(e)]. As confirmed from Fig. 16.13(a), the charge density distribution of $Q_{\mathrm{I}, \mathrm{a}}$ is oriented along the $b$ axis, increasing the polarizability and the resulting $\varepsilon_{2}$ value significantly in this direction. Consequently, $\varepsilon_{b}$ of $\alpha-\mathrm{FAPbI}_{3}$ exhibits the maximum $\varepsilon_{2}$ value, while very low $Q_{\mathrm{I}, \mathrm{c}}$ results in very small $\varepsilon_{\mathrm{c}}$. In the case of $\mathrm{MAPbI}_{3}$, the distribution of $Q_{\mathrm{I}, \mathrm{a}}$ is aligned along the $a$ axis, leading to the higher $\varepsilon_{\mathrm{a}}$ value at $2.46 \mathrm{eV}$ ( $E_{1}$ transition), compared with $\varepsilon_{\mathrm{b}}$ and $\varepsilon_{\mathrm{c}}$ [Fig. 16.9(d)]. Moreover, the similar $\varepsilon_{2}$ values of $\varepsilon_{\mathrm{b}}$ and $\varepsilon_{\mathrm{c}}$ are consistent with the corresponding charge densities $\left(Q_{\mathrm{I}, \mathrm{b}} \sim Q_{\mathrm{I}, \mathrm{c}}\right)$ shown in Fig. 16.13(b). In contrast, $\mathrm{CsPbI}_{3}$ shows negligible optical anisotropy because of the uniform charge distribution of $Q_{\mathrm{I}, \mathrm{a}-\mathrm{c}}$. Moreover, when the $\mathrm{N}$ atom position of $\mathrm{MA}^{+}$is shifted intentionally and is placed at the center position of the $\mathrm{C}-\mathrm{N}$ bond in Fig. 16.13(b), the charge density distribution of $-0.6 \sim-0.4 \mathrm{eV}$ becomes more uniform and the strong anisotropic behavior disappears [27]. Accordingly, the anti-coupling effect is induced primarily by the $\mathrm{I} \cdots \mathrm{H}-\mathrm{N}$ hydrogen bonding interaction.

In Fig. 16.9, among the $\varepsilon_{2}$ spectra calculated for different light polarizations, the $\varepsilon_{2}$ spectrum having the lowest $\varepsilon_{2}$ values in the visible region shows the best match with the experimental data. This result indicates that the visible-light absorption in the actual hybrid perovskites is modified significantly by the strong anti-coupling effect. In other words, although the $\mathrm{Pb}$-derived conduction band $\left(C_{1}\right)$ is independent of the A species, the electronic states of the I atoms are influenced strongly by the hydrogen bonding of the A-site cation.

Figure 16.14 summarizes the dielectric functions of (a) $\alpha$-FAPbX 3 , (b) $\mathrm{MAPbX}_{3}$ and (c) $\mathrm{CsPbX}_{3}(\mathrm{X}=\mathrm{I}, \mathrm{Br}, \mathrm{Cl})$ obtained from DFT calculations [27]. These dielectric functions have been obtained by replacing the A-site cation in identical $\mathrm{PbI}_{3}{ }^{-}, \mathrm{PbBr}_{3}{ }^{-}$ and $\mathrm{PbCl}_{3}{ }^{-}$structures that are deduced from the structural optimization of $\mathrm{MAPbX}_{3}$. In this figure, however, only the $\varepsilon_{2}$ spectra of $\left.\varepsilon_{\mathrm{c}}(\alpha-\mathrm{FAPbX})_{3}\right)$ and $\varepsilon_{\mathrm{b}}\left(\mathrm{MAPbX}_{3}\right.$ and $\left.\mathrm{CsPbX}_{3}\right)$ are shown, and the bars indicate the energy positions of the $E_{1}$ transition at the $M_{2}$ point $\left(V_{1} C_{1}\right)$. It should be noted that, when the $\mathrm{PbX}_{3}^{-}$structures derived from $\mathrm{MAPbX}_{3}$ are assumed, the energies of the $E_{1}$ and $E_{2}$ transitions in $\alpha-\mathrm{FAPbX}_{3}$ become almost identical. It can be seen that the shapes of the $\varepsilon_{2}$ spectra are similar when the $\varepsilon_{2}$ spectra of the same $X$ are compared. Nevertheless, the $\varepsilon_{2}$ amplitude varies systematically with the A-site cation, independent of $X$, due to the presence of the anti-coupling effect. In particular, the $\varepsilon_{2}$ value of the $E_{1}$ transition, indicated by the bar, increases notably as the hydrogen bonding interaction becomes weaker.

For the variation of the $\varepsilon_{2}$ amplitude with the A-site cation, more quantitative analyses have been made. Figure 16.15 shows the $\varepsilon_{2}$ values for the $E_{1}$ transitions in 

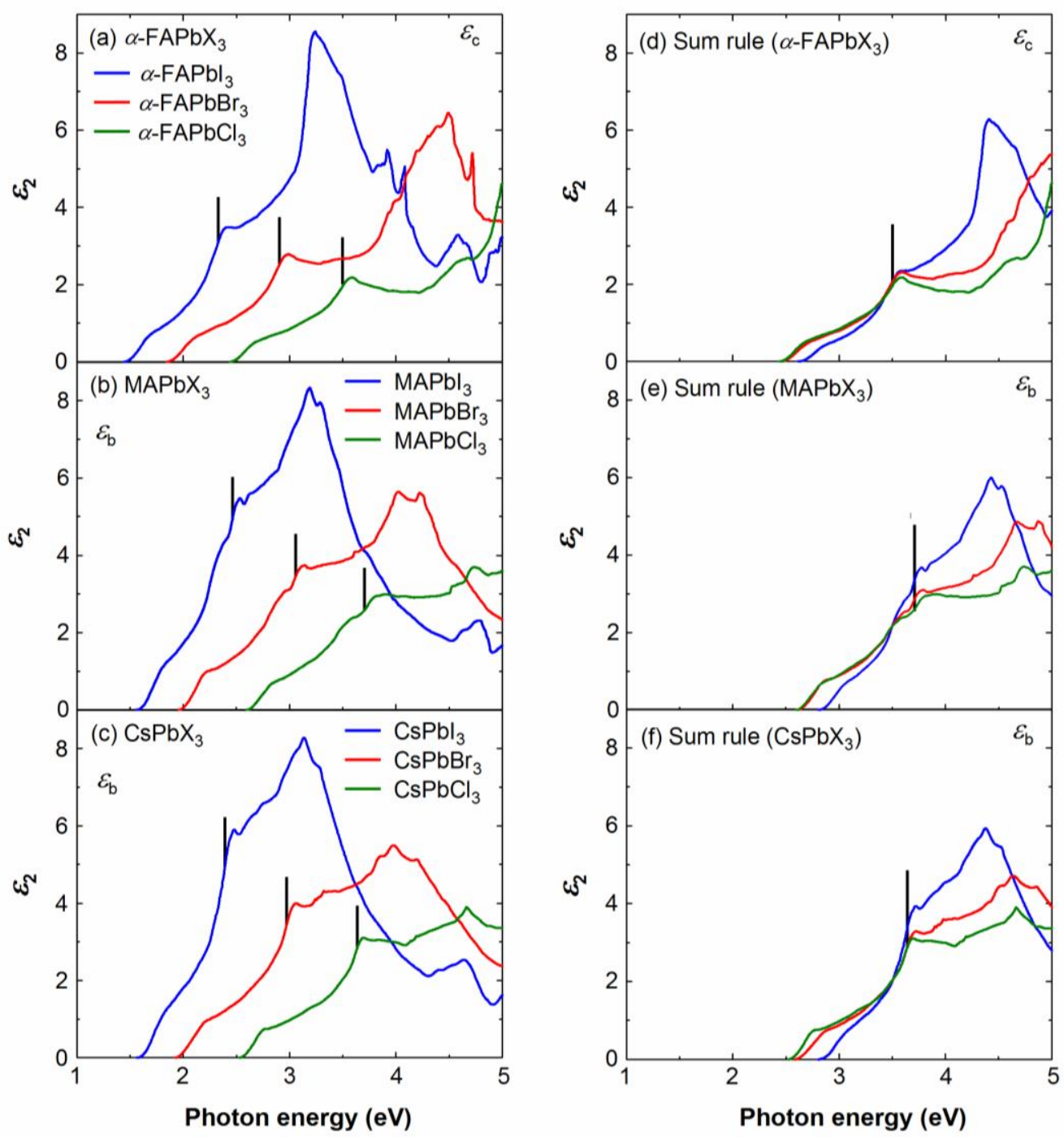

Fig. 16.14 $\varepsilon_{2}$ spectra of (a) $\alpha-\mathrm{FAPbX}_{3}$, (b) $\mathrm{MAPbX}_{3}$, and (c) $\mathrm{CsPbX}_{3}(\mathrm{X}=\mathrm{I}, \mathrm{Br}, \mathrm{Cl})$ calculated from DFT, together with the corresponding $\varepsilon_{2}$ spectra of (d) $\alpha$-FAPbX $\mathrm{MAPbX} 3$, and (f) $\mathrm{CsPbX}_{3}$ obtained by applying the sum rule for $\mathrm{APbI}_{3}$ and $\mathrm{APbBr}_{3}$. In the figures, only the $\varepsilon_{2}$ spectra for $\varepsilon_{\mathrm{c}}\left(\alpha-\mathrm{FAPbX}_{3}\right)$ and $\varepsilon_{\mathrm{b}}\left(\mathrm{MAPbX}_{3}\right.$ and $\left.\mathrm{CsPbX}\right)$ are shown. The bars indicate the energy positions of the $E_{1}$ transition at the $M_{2}$ point $\left(V_{1} C_{1}\right)$. The $\varepsilon_{2}$ spectra of (a)-(c) have been obtained by replacing the A-site cation in the identical $\mathrm{PbX}_{3}{ }^{-}$structures determined from the structural optimization of $\mathrm{MAPbX}_{3}$. The data reported in Ref. [27] are shown.

$\mathrm{APbX}_{3}$, which corresponds to the $\varepsilon_{2}$ values indicated by the bars in Figs. 16.14(a)-(c), as a function of the valence charge ratio expressed by $Q_{\mathrm{X}} / Q_{\text {Total }}$ [27]. Here, $Q_{\mathrm{X}} / Q_{\text {Total }}$ 


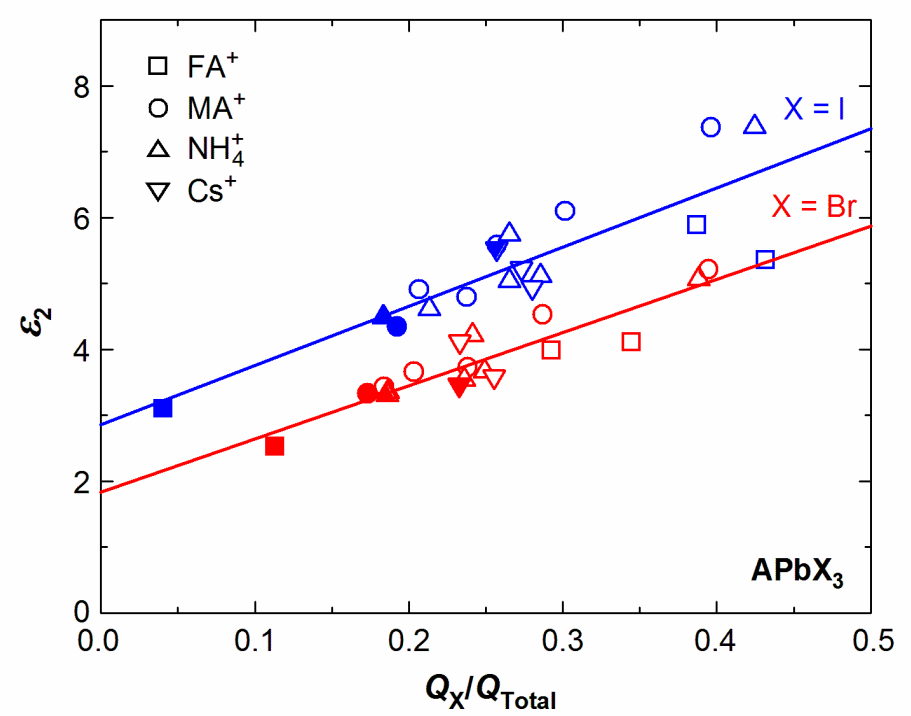

Fig. 16.15 (a) $\varepsilon_{2}$ values for the $E_{1}$ transition at the $M_{2}$ point $\left(V_{1} C_{1}\right)$ in $\mathrm{APbX}_{3}\left(\mathrm{~A}=\mathrm{FA}^{+}\right.$, $\left.\mathrm{MA}^{+}, \mathrm{NH}_{4}{ }^{+}, \mathrm{Cs}^{+} ; \mathrm{X}=\mathrm{I}, \mathrm{Br}\right)$ as a function of the valence charge ratio expressed by $Q_{\mathrm{X}} / Q_{\text {Total. }}$. The $Q_{\mathrm{X}}$ represents the valence charge of the X-site atom along the $a, b$, and $c$ axes and is calculated by selecting an energy region that corresponds to the $E_{1}$ transition at the $M_{2}$ point $\left(V_{1} C_{1}\right)$, whereas $Q_{\text {Total }}$ denotes the total charge of $\mathrm{APbX}_{3}$ in the same energy region. The closed symbol shows the plot for the lowest $Q_{\mathrm{x}} / Q_{\text {Total }}$ in each $\mathrm{APbX}_{3}$. The data reported in Ref. [27] are shown.

represents the relative magnitude of each $\mathrm{X}$-site valence charge (i.e., $Q_{\mathrm{X}, \mathrm{a}}, Q_{\mathrm{X}, \mathrm{b}}$ or $Q_{\mathrm{X}, \mathrm{c}}$ ) with respect to the total charge of $\mathrm{APbX}_{3}\left(Q_{\text {Total }}\right)$. For the calculation of $Q_{\mathrm{X}} / Q_{\text {Total }}$, the energy ranges that correspond to the $E_{1}$ transitions have been selected. In Fig. 16.15, the results for $\varepsilon_{\mathrm{a}}, \varepsilon_{\mathrm{b}}$ and $\varepsilon_{\mathrm{c}}$ in each $\mathrm{APbX}_{3}$ are plotted and the solid lines show the linear fitting results. It can be seen that the $\varepsilon_{2}$ value increases linearly with $Q_{\mathrm{X}} / Q_{\text {Total }}$, confirming the presence of a universal rule for the effect of the A-site cation. As a result, the $\varepsilon_{2}$ value of $\varepsilon_{\mathrm{a}}, \varepsilon_{\mathrm{b}}$ or $\varepsilon_{\mathrm{c}}$ in $\mathrm{APbX}_{3}$ increases when the corresponding $Q_{\mathrm{X}, \mathrm{a}}, Q_{\mathrm{X}, \mathrm{b}}$ or $Q_{\mathrm{X}, \mathrm{c}}$ becomes larger,

The closed symbol in Fig. 16.15 shows the $\varepsilon_{2}$ value for the lowest $Q_{x} / Q_{\text {Total }}$ in each $\mathrm{APbX}_{3}$. As we have seen in Fig. 16.9, the experimental $\varepsilon_{2}$ values are reproduced when the strong anti-coupling effect is considered (i.e., the lowest $\varepsilon_{2}$ values in the DFT spectra). Accordingly, the $\varepsilon_{2}$ values of the closed symbols approximate the light absorption strengths in the hybrid perovskites. These $\varepsilon_{2}$ values lead to a straightforward conclusion; the anti-coupling interaction strengthens with increasing number of $\mathrm{N}$ atoms 
in the center cation. Since $\mathrm{FA}^{+}$has two $\mathrm{N}$ atoms, a quite large anti-coupling interaction occurs in $\alpha$-FAPbX 3 , which in turn reduces the visible-light absorption significantly, as observed in the experimental $\alpha$ spectra [see Fig. 16.5(b)]. In contrast, $\mathrm{Cs}^{+}$is stabilized at the center position of the $\mathrm{PbX}_{3}{ }^{-}$cage [Fig. 16.13(c)] and shows the weak anti-coupling effect. Consequently, the visible light absorption increases in the order $\mathrm{FA}^{+}<\mathrm{MA}^{+}<$ $\mathrm{Cs}^{+}$.

As confirmed from Fig. 16.9, the experimental $\varepsilon_{2}$ spectra of $\alpha-\mathrm{FAPbI}_{3}, \mathrm{MAPbI}_{3}$ and $\mathrm{MAPbBr}_{3}$ do not match those calculated assuming optical isotropy [i.e., $\left(\varepsilon_{\mathrm{a}}+\varepsilon_{\mathrm{b}}+\varepsilon_{\mathrm{c}}\right) / 3$ ] and the overall visible absorption in these perovskites is minimized by the anti-coupling interaction. As mentioned earlier, $\mathrm{MA}^{+}$and $\mathrm{FA}^{+}$in the $\mathrm{PbI}_{3}{ }^{-}$reorient rapidly with a time scale of $0.5-14$ ps at room temperature [19,34-40]. In the DFT calculation that assumes $0 \mathrm{~K}$, however, the cation position is fixed completely and the effect of the cation reorientation is neglected. Even in this case, excellent agreement can be seen between the experimental and DFT results. This result supports the fact that the N-I distance is close and the anti-coupling phenomenon persists even at room temperature. In particular, the molecular dynamics (MD) simulations reveal that the orientation of the center cation is not completely random [35-40], and $\mathrm{MA}^{+}$and $\mathrm{FA}^{+}$are preferentially directed toward the face center position of the cubic structure $[36,37,39,40]$ by maintaining the stable $\mathrm{I}-\mathrm{H}$ (or I-N) distance even at elevated temperature [36,38,39,40]. Accordingly, the anti-coupling effect observed in the room-temperature optical spectrum can be interpreted by the strong hydrogen bonding effect of $\mathrm{MA}^{+}$and $\mathrm{FA}^{+}$.

On the other hand, the optical effect of $\mathrm{X}$ on the $\varepsilon_{2}$ spectrum of hybrid perovskites can be modeled according to the sum rule [27], given by the following expression [78]:

$$
\int E \varepsilon_{2}(E) d E=\text { const. }
$$

When the $\varepsilon_{2}$ spectrum is shifted toward higher energy by $\Delta E$, the sum rule requires that the $\varepsilon_{2}$ peak amplitude reduces to $E /(E+\Delta E)$ because $\int E \varepsilon_{2}(E) d E=$ $\int\left(E+\Delta E_{\mathrm{g}}\right) \varepsilon_{2}\left(E+\Delta E_{\mathrm{g}}\right) d E$. As confirmed from Fig. 16.14(a)-(c), with decreasing halogen mass $(\mathrm{I}>\mathrm{Br}>\mathrm{Cl})$, the whole $\varepsilon_{2}$ spectrum shifts toward higher energy and the $\varepsilon_{2}$ amplitude decreases. Such spectral changes induced by the $\mathrm{X}$ replacement can be expressed by applying Eq. (16.1). Figure 16.14 shows the $\varepsilon_{2}$ spectra of (d) $\alpha$-FAPbX 3 , (e) $\mathrm{MAPbX}_{3}$ and (f) $\mathrm{CsPbX}_{3}$ obtained from the calculations assuming the sum rule [27]. In these results, the $\varepsilon_{2}$ spectra of $\mathrm{APbI}_{3}$ and $\mathrm{APbBr}_{3}$ are shifted so that the $E_{1}$ transition energies match those of $\mathrm{APbCl}_{3}$. In this case, the amplitude of the shifted $\varepsilon_{2}$ spectra is 
reduced further by applying the sum rule. Under these assumptions, the $\varepsilon_{2}$ values for the $E_{1}$ transition become similar and all the spectra show reasonable overlap in a low energy region $(<3.7 \mathrm{eV})$. Accordingly, the $\varepsilon_{2}$ amplitude variation induced by $\mathrm{X}$ can simply be interpreted by the change in the transition energy.

\subsection{Operating Principles of Hybrid Perovskite Solar Cells}

In this section, to understand the working principles of hybrid perovskite solar cells, we will examine the photocarrier generation/collection in $\mathrm{MAPbI}_{3}$ solar cells based on (i) the internal quantum efficiency (IQE) simulation of a standard solar cell [26], performed by the optical admittance method assuming a perfectly flat structure (Section 2.2 in Vol. 2) and (ii) the characterization of the optical and recombination losses in $\mathrm{MAPbI}_{3}$ solar cells [87], determined from the EQE analysis using the e-ARC method (Section 2.3 in Vol. 2). These IQE and EQE calculations have been performed using the $\mathrm{MAPbI}_{3}$ optical constants shown in Fig. 16.5 (see also Fig. 10.2 in Vol. 2).

\subsubsection{Optical Simulation of a $\mathrm{CH}_{3} \mathrm{NH}_{3} \mathrm{PbI}_{3}$ Solar Cell}

Figure 16.16(a) shows the IQE and internal absorptance (IA) spectra for a $\mathrm{MAPbI}_{3}$ solar cell consisting of glass $/ \mathrm{SnO}_{2}: \mathrm{F} \quad(600 \quad \mathrm{~nm}) / \mathrm{TiO}_{2} \quad(200 \mathrm{~nm}) / \mathrm{MAPbI}_{3} \quad(400$ $\mathrm{nm}) /$ spiro-OMeTAD $(500 \mathrm{~nm}) / \mathrm{Ag}$ [26]. The IQE and IA spectra can be calculated by $I Q E=E Q E /(1-R)$ and $I A=A /(1-R)$, where $R$ and $A$ represent the reflectance and absorptance of the solar cell structure, respectively. By assuming a perfectly flat structure, the $R$ and EQE spectra can be calculated directly using Eqs. (2.18) in Vol. 2 and (2.26) in Vol. 2, respectively. In the optical simulation of Fig. 16.16(a), the effect of the multiple light reflection/transmission within the glass substrate is neglected (Section 2.2.2 in Vol. 2) and 100\% collection efficiency of generated carriers is assumed for the perovskite layer. This simulation result can be reproduced by using the optical constants of glass (Fig. 13.1 in Vol. 2), $\mathrm{TiO}_{2}$ (Fig. 11.12 in Vol. 2), $\mathrm{MAPbI}_{3}$ (Fig. 10.2 in Vol. 2), spiro-OMeTAD (Fig. 9.18 in Vol. 2) and Ag (Fig. 12.6 in Vol. 2) summarized in Part II (Vol. 2) of this book. For the $\mathrm{SnO}_{2}: \mathrm{F}$ layer [26], however, we assume the Drude parameters of $A_{\mathrm{D}}=0.919 \mathrm{eV}$ and $\Gamma=0.129 \mathrm{eV}$, which correspond to $N_{\mathrm{opt}}=1.2 \times 10^{20}$ $\mathrm{cm}^{-3}$ and $\mu_{\mathrm{opt}}=49 \mathrm{~cm}^{2} /(\mathrm{Vs})$ (see Section 18.3.4), together with the band-edge Tauc-Lorentz parameters of $A=44.55 \mathrm{eV}, C=12.00 \mathrm{eV}, E_{\mathrm{g}}=3.30 \mathrm{eV}$ and $E_{0}=7.00 \mathrm{eV}$ [see Eqs. (11.1) in Vol. 2 and (11.2) in Vol. 2]. The above calculation shows that the average $R$ value in the visible region $(\lambda=400 \sim 700 \mathrm{~nm}$ ) is $\sim 15 \%$ and a strong 
(a)

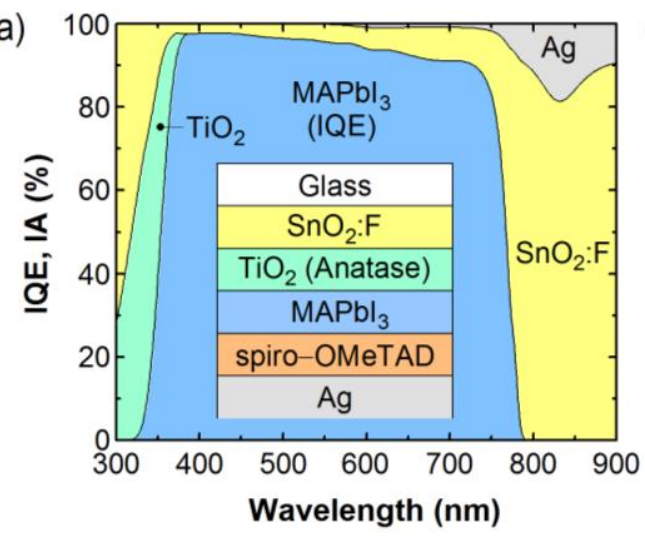

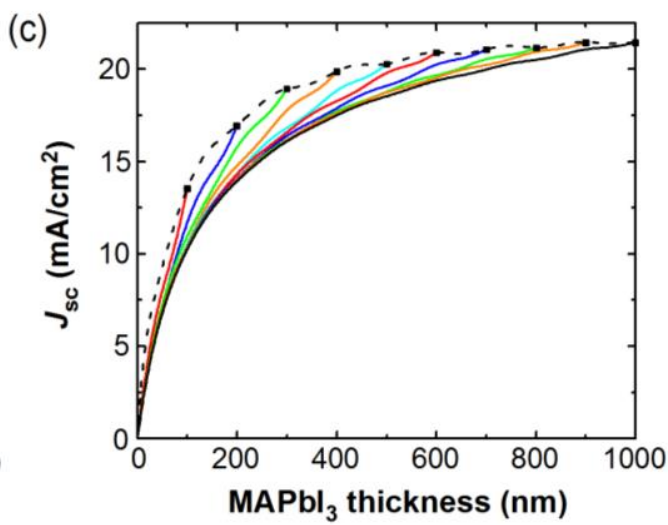

(b)

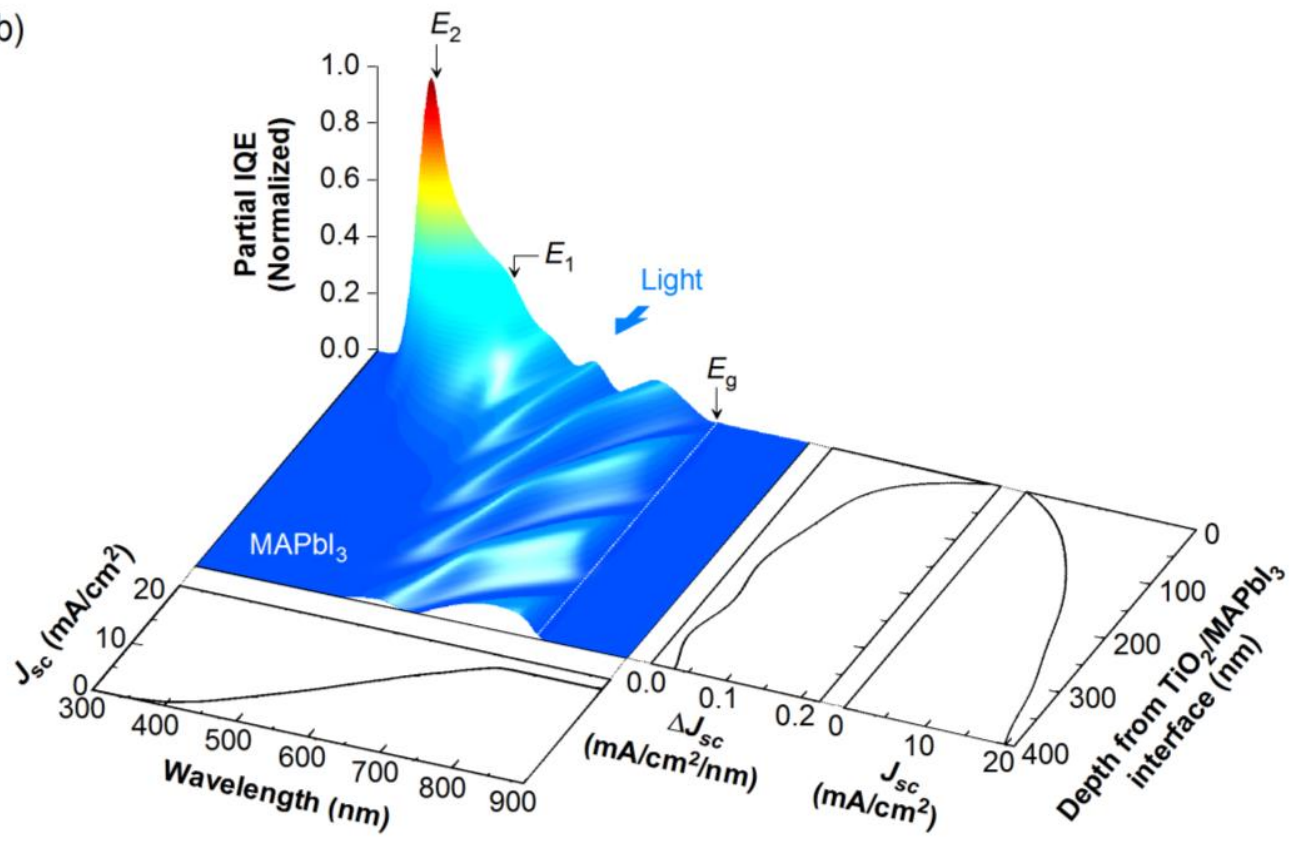

Fig. 16.16 (a) IQE spectrum of the $\mathrm{MAPbI}_{3}$ layer and IA spectra of the component layers in the glass $/ \mathrm{SnO}_{2}: \mathrm{F}(600 \mathrm{~nm}) / \mathrm{TiO}_{2}(200 \mathrm{~nm}) / \mathrm{MAPbI}_{3}(400 \mathrm{~nm}) /$ spiro-OMeTAD (500 nm)/Ag structure [26], (b) normalized partial IQE spectra for the $\mathrm{MAPbI}_{3}$, and (c) integrated $J_{\mathrm{sc}}$ for perovskite solar cells with discrete $\mathrm{MAPbI}_{3}$ thicknesses from 100 to $1000 \mathrm{~nm}$ [26]. In (b), $\Delta J_{\text {sc }}$ shows the $J_{\text {sc }}$ component generated within the 1-nm-thick $\mathrm{MAPbI}_{3}$ sublayer, and integrated $J_{\mathrm{sc}}$ values relative to the depth from the $\mathrm{TiO}_{2}$ interface and $\lambda$ are also shown. The arrows in (b) show the $E_{\mathrm{g}}\left(E_{0}\right), E_{1}$, and $E_{2}$ positions. In (c), the solid circles denote the end points of the $\mathrm{MAPbI}_{3}$ thickness and the dotted line shows the $J_{\mathrm{sc}}$ values obtained by varying the $\mathrm{MAPbI}_{3}$ layer thickness in the solar cell. 
interference effect appears in the $R$ and EQE spectra due to the assumed flat structure.

As shown in Fig. 16.16(a), for the $\mathrm{SnO}_{2}: \mathrm{F}$, the strong light absorption occurs at $E \geq$ $3.3 \mathrm{eV}(\lambda \leq 375 \mathrm{~nm})$ due to the interband transition, whereas the light absorption at $\lambda>$ $375 \mathrm{~nm}$ indicates the contribution by the free carrier absorption (Chapter 18). The $\mathrm{TiO}_{2}$ layer shows the interband transition at $\lambda<385 \mathrm{~nm}$. Since $E_{\mathrm{g}}$ of spiro-OMeTAD $(2.95$ $\mathrm{eV}$ ) is higher than that of $\mathrm{MAPbI}_{3}$, the light absorption in this layer is negligible. In addition, the light absorption in the $\mathrm{Ag}$ back electrode occurs mainly in the $\lambda$ region below $E_{\mathrm{g}}$ of $\mathrm{MAPbI}_{3}$ due to the strong light transmission in this regime. In Fig. 16.16(a), IQE of $\mathrm{MAPbI}_{3}$ is quite high in a wide $\lambda$ region of $400-700 \mathrm{~nm}$ and the IQE maximum in this range is limited by the free carrier absorption of the $\mathrm{SnO}_{2}: \mathrm{F}$ layer.

Under AM1.5G conditions, $J_{\mathrm{sc}}$ of the solar cell is $19.9 \mathrm{~mA} / \mathrm{cm}^{2}$ with a reflection loss of $3.6 \mathrm{~mA} / \mathrm{cm}^{2}$ and parasitic optical losses of $1.3 \mathrm{~mA} / \mathrm{cm}^{2}\left(\mathrm{SnO}_{2}: \mathrm{F}\right), 0.3 \mathrm{~mA} / \mathrm{cm}^{2}\left(\mathrm{TiO}_{2}\right)$, and $0.1 \mathrm{~mA} / \mathrm{cm}^{2}(\mathrm{Ag})$ in the energy region above $E_{\mathrm{g}}=1.61 \mathrm{eV}\left(\mathrm{MAPbI}_{3}\right)$. When a natural texture is present, the front light reflection is suppressed and $J_{\mathrm{sc}}$ of the $\mathrm{MAPbI}_{3}$ solar cell increases further.

To visualize carrier generation within the $\mathrm{MAPbI}_{3}$ layer, partial IQE spectra are calculated by dividing the $\mathrm{MAPbI}_{3}$ layer into 400 sublayers (i.e., 1-nm thick sublayer). In Fig. 16.16(b), the partial IQE calculated for different depths from the interface and wavelengths are shown. These IQE values are normalized relative to the maximum IQE value and, if the partial IQE spectra are integrated, the IQE spectrum of Fig. 16.16(a) can be obtained. The arrows in Fig. 16.16(b) denote the $\lambda$ positions that correspond to the $E_{\mathrm{g}}\left(E_{0}\right), E_{1}$ and $E_{2}$ transitions. In the $E_{2}$ transition regime, the partial IQE is limited by light absorption in the upper $\mathrm{SnO}_{2}: \mathrm{F}$ and $\mathrm{TiO}_{2}$ layers. At $E \geq E_{1}$, the partial IQE exhibits rapid decay with increasing depth because of strong light absorption within $\mathrm{MAPbI}_{3}$. In contrast, in the region of $E_{0} \leq E<E_{1}$, the IQE values are low because of the smaller $\alpha$ values and photocarriers are generated uniformly throughout the entire $\mathrm{MAPbI}_{3}$ layer with the appearance of the optical interference effect. In this region, the electrons and holes that are generated near the front and rear interfaces, respectively, need to travel through the whole $\mathrm{MAPbI}_{3}$ layer. Therefore, the high $J_{\mathrm{sc}}$ of $20 \mathrm{~mA} / \mathrm{cm}^{2}$ observed experimentally supports the efficient carrier collection within the whole absorber layer.

In Fig. 16.16(b), $\Delta J_{\text {sc }}$ shows the $J_{\text {sc }}$ component generated within the 1-nm-thick $\mathrm{MAPbI}_{3}$ sublayer, and the integrated $J_{\text {sc }}$ values relative to the depth are also shown. The integrated values obtained for each $\lambda$ are also indicated. The calculation result of $\Delta J_{\mathrm{sc}}$ confirms the intense carrier generation near the $\mathrm{TiO}_{2}$ interface and $J_{\mathrm{sc}}$ increases gradually at larger depth by the carrier collection in the low $\alpha$ region $\left(E_{0} \leq E<E_{1}\right)$. It 
should be emphasized that the contribution of $J_{\mathrm{sc}}$ at $\lambda \geq 500 \mathrm{~nm}$ accounts for $73 \%$ of the total $J_{\mathrm{sc}}$, confirming that the longer wavelength response is critical to realize a high $J_{\mathrm{sc}}$ [47].

Figure 16.16(c) summarizes the change in $J_{\mathrm{sc}}$ with $\mathrm{MAPbI}_{3}$ thickness [26]. The solid lines indicate the integrated $J_{\mathrm{sc}}$ estimated from $\mathrm{MAPbI}_{3}$ layers with discrete thicknesses ranging from 100 to $1000 \mathrm{~nm}$, and the end points are shown by the solid circles. The dotted line in this figure shows the $J_{\mathrm{sc}}$ values obtained by changing the $\mathrm{MAPbI}_{3}$ layer thickness. In Fig. 16.16(c), the difference in $J_{\text {sc }}$ observed between the dotted line and the solid line for the $1000 \mathrm{~nm}$ thickness indicates the influence of the back-side reflection. When the absorber thickness is $400 \mathrm{~nm}$, for example, the contribution of $J_{\text {sc }}$ improved by the back-side reflection is $2.4 \mathrm{~mA} / \mathrm{cm}^{2}$. Accordingly, the effect of the back-side reflection is relatively large in the hybrid solar cell and the light is absorbed effectively even in the thin layers. The optimum thickness of $400 \mathrm{~nm}$ confirmed in Fig. 16.16(c) can therefore be interpreted as a consequence of the optical confinement effect. The enhanced light absorption is particularly necessary for tandem-type solar cells and the EQE simulation of a $\mathrm{MAPbI}_{3}$ top cell confirms the importance of the strong optical confinement in the long-wavelength regime (Fig. 6.8).

\subsubsection{Carrier Loss Mechanisms}

The optical and recombination losses in $\mathrm{MAPbI}_{3}$ solar cells can be evaluated from the EQE analyses using the e-ARC method (Section 2.3 in Vol. 2). The ARC method is a calculation method developed originally for the estimation of the optical losses in submicron-textured solar cells, whereas the extended ARC (e-ARC) method further allows the analysis of the recombination loss. Here, from the recombination losses deduced from the e-ARC analyses [87], carrier recombination dynamics in $\mathrm{MAPbI}_{3}$ solar cells are discussed.

Figure 16.17 shows the optical models for $\mathrm{MAPbI}_{3}$ solar cells (a) with an HTL [88] and (b) with no HTL [89], and the corresponding EQE analysis results are shown in (c) and (d), respectively [87]. The solar cell with the HTL has a structure of glass $/ \mathrm{SnO}_{2}: \mathrm{F} / \mathrm{TiO}_{2} / \mathrm{MAPbI}_{3} /$ polytriarylamine(PTAA)/Au and shows a high efficiency of $17.6 \%\left(V_{\mathrm{oc}}=1.1 \mathrm{~V}, J_{\mathrm{sc}}=20.5 \mathrm{~mA} / \mathrm{cm}^{2}\right.$ and $\left.F F=0.78\right)$ [88]. This device incorporates the PTAA layer as the HTL and has a simple structure without a mesoporous $\mathrm{TiO}_{2}$ layer. In the optical model of Fig. 16.17(a), a complicated structure of $\mathrm{SnO}_{2}: \mathrm{F} / \mathrm{SiO}_{2} / \mathrm{SnO}_{2} /$ glass (TEC-8, see Fig. 18.25$)$ has been simplified to a $\mathrm{SnO}_{2}: \mathrm{F}(600$ 
(a)

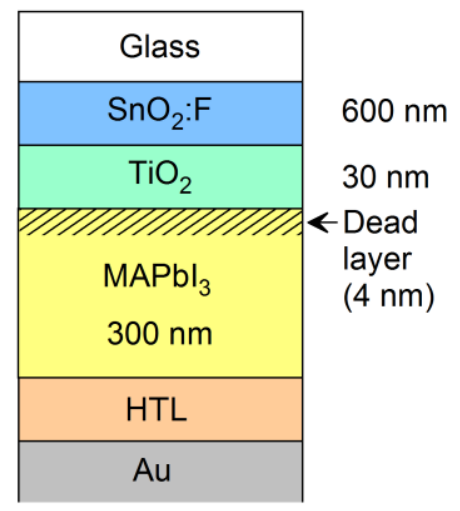

(b)

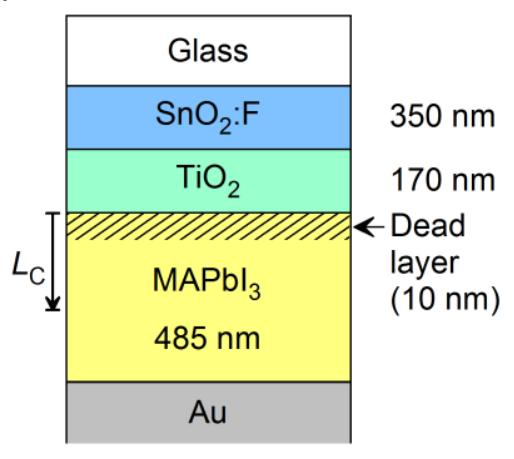

(c)

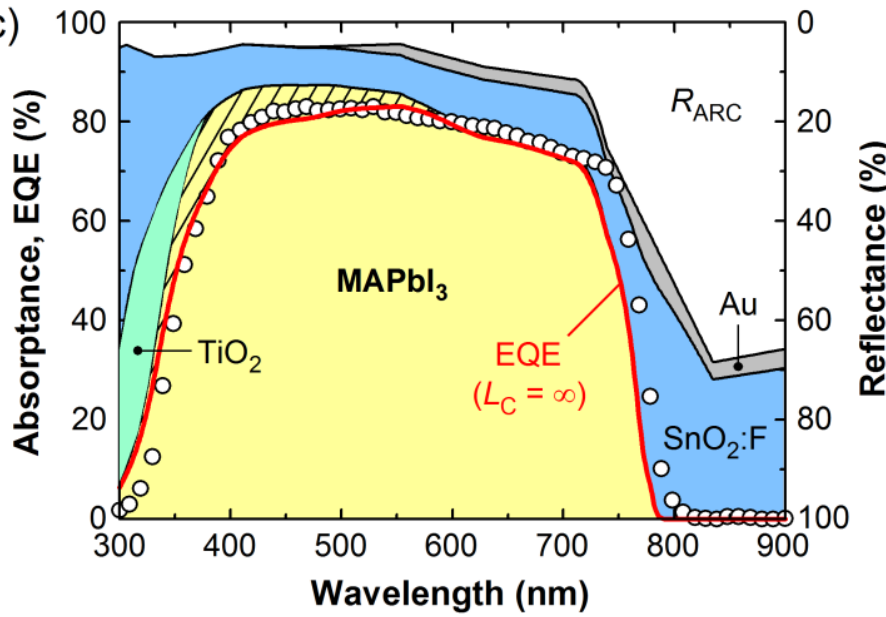

(d)

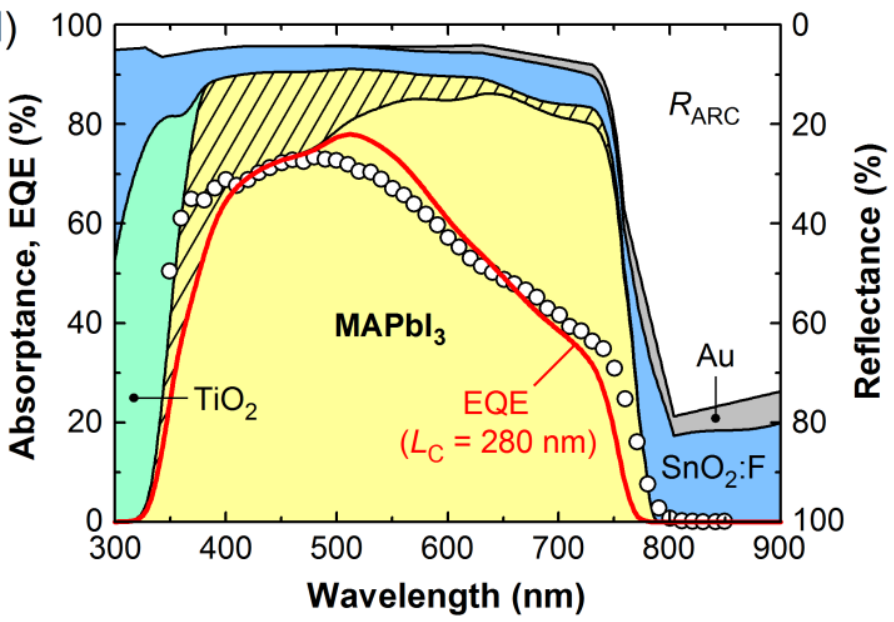

Fig. 16.17 Optical models for $\mathrm{MAPb}_{3}$ solar cells (a) with an HTL [88] and (b) with no HTL [89], and the corresponding EQE analysis results for the $\mathrm{MAPbI}_{3}$ solar cells (c) with the HTL and (d) with no HTL. In (a) and (b), the values represent the layer thicknesses in the optical model, and the thickness of the HTL layer in (a) is assumed to be zero. For the $\mathrm{MAPbI}_{3}$ layers, the presence of optical dead layers near the $\mathrm{TiO}_{2}$ interfaces is assumed. In (c) and (d), the EQE analyses are carried out based on the e-ARC method using the carrier collection length $\left(L_{\mathrm{C}}\right)$ as a fitting parameter. The calculated EQE spectra are shown by red lines. The hatched-line regions show the EQE losses originating from the front dead layers. The analysis data are taken from Ref. [87].

$\mathrm{nm}$ )/glass structure [26] and the layer thicknesses were deduced directly from the SEM images. In the optical model, however, the PTAA layer thickness is assumed to be zero, since the optical constants of the PTAA layer are unknown and the optical effect of the 
wide-gap PTAA layer $\left(E_{\mathrm{g}} \sim 3 \mathrm{eV}\right)$ is negligible. For the calculation, the optical constants of $\mathrm{SnO}_{2}: \mathrm{F}$ (Fig. 11.11. in Vol. 2), $\mathrm{TiO}_{2}$ (Fig. 11.12 in Vol. 2), $\mathrm{MAPbI}_{3}$ (Fig. 10.2 in Vol. 2) and $\mathrm{Au}$ (Fig. 12.8 in Vol. 2) have been used. In the EQE analyses, the carrier recombination in the $\mathrm{TiO}_{2} / \mathrm{MAPbI}_{3}$ interface region is further assumed. This recombination is modeled by simply dividing a $\mathrm{MAPbI}_{3}$ bulk layer into two layers and treating a thin layer located at the $\mathrm{TiO}_{2}$ interface as a "dead layer" that allows no carrier extraction [see Fig. 16.17(a)].

In Fig. 16.17(c), the absorptance spectra of the solar-cell component layers calculated using the optical model of Fig. 16.17(a) are shown. The $R_{\mathrm{ARC}}$ shows the reflectance deduced from the ARC method, and the $R_{\mathrm{ARC}}$ spectrum includes the contribution of light scattering generated in the textured solar cell (see Section 2.2.3 in Vol. 2). In this figure, the yellow-colored region shows the light absorption in the $\mathrm{MAPbI}_{3}$, and the hatched-line region corresponds to the EQE loss caused by the front dead layer. The red line indicates the EQE spectrum calculated by e-ARC, from which the carrier collection length $\left(L_{\mathrm{C}}\right)$ can be determined. In the EQE analysis using the e-ARC method, the $L_{\mathrm{C}}$ value modeled by $\left.H(\lambda)=1-\exp \left[-\alpha(\lambda) L_{\mathrm{C}}\right)\right]$ is used as a fitting parameter, where $H(\lambda)$ and $\alpha(\lambda)$ are the carrier collection efficiency and absorption coefficient spectrum of the absorber layer, respectively. In this simple model, $L_{C}$ shows a meaningful value only when $L_{C}$ is smaller than the layer thickness and we obtain $L_{C}=\infty$ when all the carriers are collected (see Fig. 2.14 in Vol. 2). In Fig. 16.17(c), the calculated EQE spectrum shows good agreement with the experimental result (open circles) when the thickness of the dead layer is $4 \mathrm{~nm}$ with $L_{\mathrm{C}}=\infty$, indicating that the dominant recombination occurs only near the $\mathrm{TiO}_{2}$ interface and the carrier loss near the HTL interface is negligible.

On the other hand, the slight disagreement of the calculated EQE, observed at $\lambda=$ $720-800 \mathrm{~nm}$, can be attributed to the calculation errors of $R_{\mathrm{ARC}}$. Specifically, when the layer thickness of solar cells is thin, the interference fringe becomes large and the calculation of $R_{\mathrm{ARC}}$ by the ARC method fails near the $E_{\mathrm{g}}$ region where $R$ shows a sharp increase. This problem can be avoided if the experimental $R$ spectrum obtained from an actual solar cell is used in Eq. (2.26) in Vol. 2. In the analysis of Fig. 16.17(c), however, the error in the $R_{\mathrm{ARC}}$ calculation affects $J_{\mathrm{sc}}$ only slightly $\left(0.4 \mathrm{~mA} / \mathrm{cm}^{2}\right)$.

The solar cell with no HTL shown in Fig. 16.17(b) has a structure of glass/SnO 2 :F/compact $\mathrm{TiO}_{2} /$ mesoporous $\mathrm{TiO}_{2}-\mathrm{MAPbI}_{3} / \mathrm{MAPbI}_{3} / \mathrm{Au}$ [89]. Quite fortunately, the optical response of the mesoporous $\mathrm{TiO}_{2}-\mathrm{MAPbI}_{3}$ mixed-phase layer can be expressed as the two separate flat layers of the $\mathrm{TiO}_{2}$ and $\mathrm{MAPbI}_{3}$ [26]. In this analysis, the $\mathrm{TiO}_{2}$ volume fraction within the mesoporous layer is assumed to be $30 \%$ (porosity $70 \%$ ). 
(a)

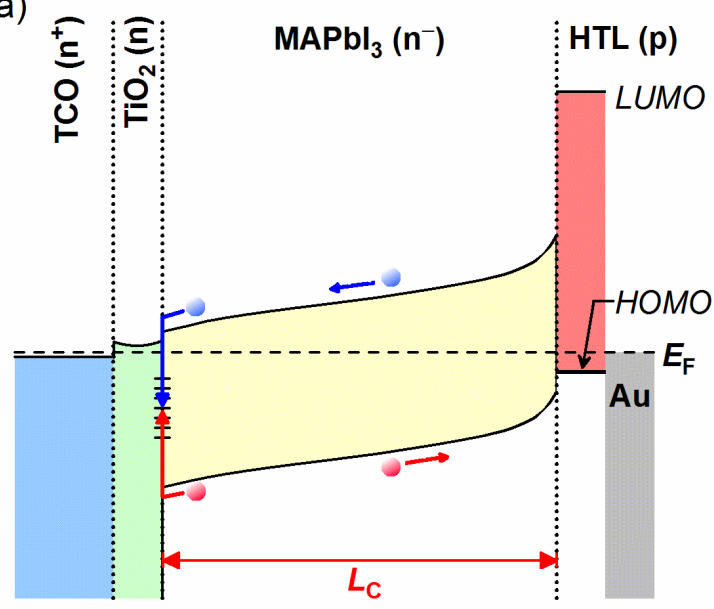

(b)

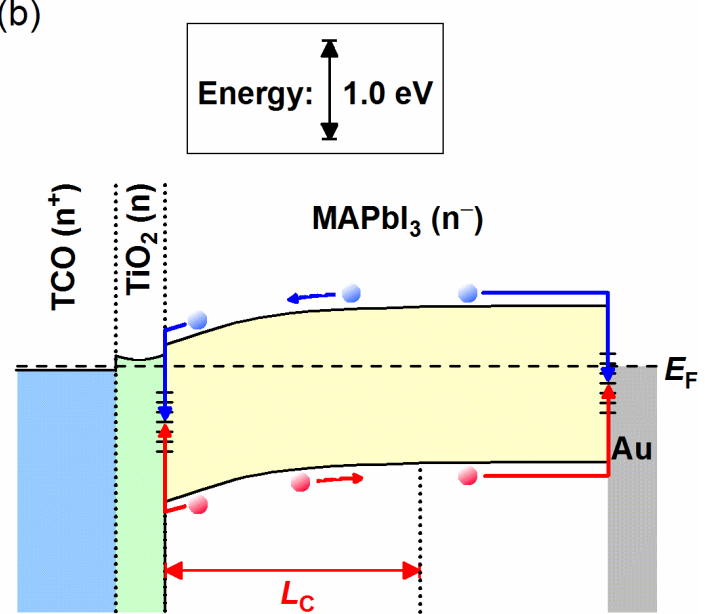

Fig. 16.18 Band diagrams proposed for $\mathrm{MAPbI}_{3}$ solar cells with an HTL (spiro-OMeTAD) and with no HTL [87]. In this figure, $L_{\mathrm{C}}$ represents the carrier collection length determined from the EQE analyses of Fig. 16.17.

Figure 16.17(d) shows the result of the EQE analysis performed for the solar cell with no HTL [87]. It can be seen that the experimental EQE spectrum of this solar cell is quite different, compared with the solar cell with the HTL. In particular, the EQE response in the longer $\lambda$ region decreases significantly when an HTL is not present [90-93]. The red line in this figure represents the EQE spectrum calculated assuming the carrier recombination in the front and rear interface regions using e-ARC. This EQE analysis reveals that the thickness of the front recombination layer is $10 \mathrm{~nm}$ with $L_{\mathrm{C}}=$ $280 \mathrm{~nm}$. Thus, the above result confirms that the carrier collection is hindered strongly when the HTL is not present and the effect of the carrier recombination appears primarily as the reduction of the EQE response in the low $\alpha$ region $(\lambda>600 \mathrm{~nm})$.

Figure 16.18 shows the band diagrams proposed for $\mathrm{MAPbI}_{3}$ solar cells (a) with an HTL (spiro-OMeTAD) and (b) with no HTL [87]. It can be seen that, in $\mathrm{MAPbI}_{3}$ solar cells, the electrons and holes generated within the absorber layer are collected in the front and rear electrodes without any potential barriers [6]. It is generally assumed that the operation of $\mathrm{MAPbI}_{3}$ solar cells occurs by the formation of a $p-i-n$ type structure [11,94-97], as confirmed by the electron-beam induced current (EBIC) [94,98] and Kelvin probe force microscopy [95] characterizations. However, the carrier type of $\mathrm{MAPbI}_{3}$ layers is basically n-type, and the Fermi level $\left(E_{\mathrm{F}}\right)$ of a standard $\mathrm{MAPbI}_{3}$ locates more closer to $\mathrm{CBM}$ with an electron concentration of $\sim 10^{17} \mathrm{~cm}^{-3}$ [99]. When 
the $\mathrm{MAPbI}_{3}$ layer shows $n$-type conductivity, the electric field is expected to concentrate at the $\mathrm{MAPbI}_{3}(n) / \mathrm{HTL}(p)$ interface with a weak electric field at the $\mathrm{TiO}_{2}(n) / \mathrm{MAPbI}_{3}(n)$ interface, as illustrated in Fig. 16.18(a). Thus, the relatively strong front-carrier recombination near the $\mathrm{TiO}_{2}$ interface can be interpreted by the weaker electric field in this region. In contrast, the carrier recombination in the solar-cell bottom region is suppressed most likely due to the presence of the strong electric field near the HTL. From the above results, the electric-field-assisted carrier collection by the formation of a $p-n^{-}-n$ type structure has been proposed for $\mathrm{MAPbI}_{3}$ solar cells [87]. The rapid degradation of the solar cell characteristics upon air exposure could be attributed, in part, to the elimination of the strong electric field in the rear $p-n$ junction region.

When the HTL is not present, however, the flat-band formation is expected to occur by the absence of the electric field in the bottom region [Fig. 16.18(b)], as evidenced by EBIC [98]. In this device, the photocarrier collection occurs through the carrier diffusion, and the intensified recombination in the rear interface region lowers the EQE response in the longer $\lambda$ region [87,92]. The lower $V_{\mathrm{oc}}$ observed in $\mathrm{MAPbI}_{3}$ solar cells with no HTL $[90-92,100]$ can be interpreted by the smaller built-in potential.

Earlier DFT studies of $\mathrm{MAPbI}_{3}$ revealed that mid-gap states are not created by the vacancy/interstitial-type defects in $\mathrm{MAPbI}_{3}$ [64,101,102], although the formation of complex defects is still possible [103]. Moreover, in the grain boundary region of $\mathrm{MAPbI}_{3}$, the $\mathrm{PbI}_{2}$-rich phase having larger $E_{\mathrm{g}}$ is formed, suppressing the carrier recombination in the grain boundary region effectively [104]. In $\mathrm{MAPbI}_{3}$ solar cells, therefore, the carrier recombination is generally considered to occur in the interface regions. Nevertheless, $V_{\mathrm{oc}}$ of $\mathrm{MAPbI}_{3}$ solar cells varies with the grain size [105], suggesting the possible recombination in the grain boundary region.

Figure 16.19 illustrates the optical and recombination losses in the $\mathrm{MAPbI}_{3}$ solar cell with an HTL [87], determined from the EQE analysis of Fig. 16.17(c). The numerical values in the figure represent the corresponding current densities in units of $\mathrm{mA} / \mathrm{cm}^{2}$. The maximum $J_{\text {sc }}$ value attainable under AM1.5G conditions is also shown and, from the ratio of output $J_{\mathrm{sc}}$ divided by the maximum attainable $J_{\mathrm{sc}}$ value, the optical gain of this solar cell is determined to be $70 \%\left(18.7 \mathrm{~mA} / \mathrm{cm}^{2}\right)$. This particular solar cell shows a large reflection loss $\left(3.2 \mathrm{~mA} / \mathrm{cm}^{2}\right)$ due to the lack of an anti-reflection coating and a rather flat layer structure with a thin absorber-layer thickness $(\sim 300 \mathrm{~nm})$. The parasitic absorption by the front TCO layer, originating mainly from free carrier absorption, reduces $J_{\mathrm{sc}}$ further by $3 \mathrm{~mA} / \mathrm{cm}^{2}$. The parasitic absorption of the TCO can be reduced largely by employing high-mobility TCO layers (Chapter 19). The recombination loss indicated in this figure corresponds to the interface recombination in the $\mathrm{TiO}_{2}$ interface 


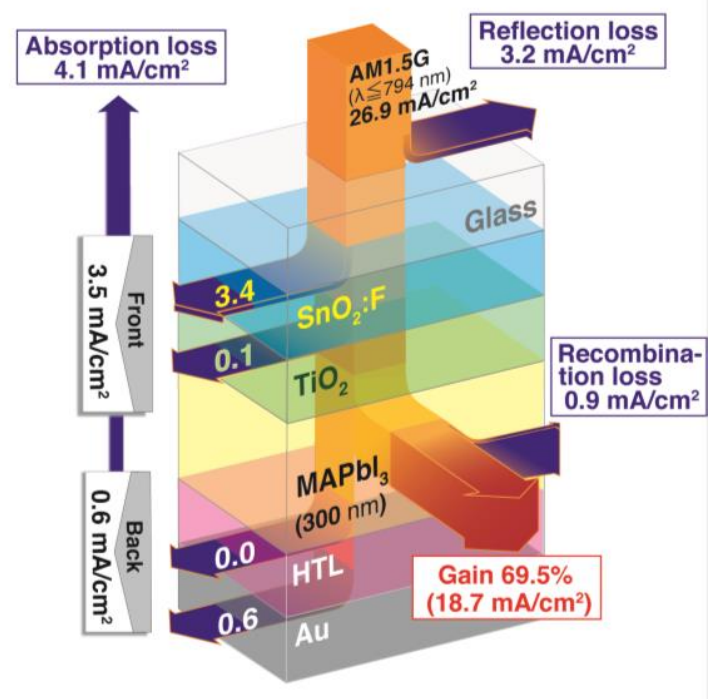

Fig. 16.19 Optical and recombination losses deduced from the EQE analysis performed for the $\mathrm{MAPbI}_{3}$ solar cell shown in Fig. 16.17(c) [87]. The numerical values represent the corresponding current densities in units of $\mathrm{mA} / \mathrm{cm}^{2}$. The maximum $J_{\mathrm{sc}}$ value attainable under AM1.5G conditions is also indicated. The optical gain shows the ratio of output $J_{\text {sc }}$ divided by the maximum attainable $J_{\text {sc }}$ value.

region.

It should be emphasized that the $\mathrm{MAPbI}_{3}$ solar cell shows quite small overall optical losses, compared with other photovoltaic devices including CuInGaSe 2 [Fig. 2.11(b) in Vol. 2], $\mathrm{Cu}_{2} \mathrm{ZnSnSe}_{4}$ [Fig. 2.15(b) in Vol. 2] and CdTe [Fig. 2.21(b) in Vol. 2] solar cells. In particular, the parasitic absorption within the $\mathrm{TiO}_{2}$ and $\mathrm{HTL}$ layers is negligible and the major optical loss occurs only in the front TCO layer, which can be optimized further. Thus, a quite high conversion efficiency reported for $\mathrm{MAPbI}_{3}$ solar cells $(\sim 20 \%)$ can be understood partly by the low parasitic absorption within the solar cell component layers [26].

As a result, the excellent performance of $\mathrm{MAPbI}_{3}$ hybrid solar cells can be interpreted as being due to a combination of (a) high $\alpha$ values comparable to those of $\mathrm{CuInGaSe}_{2}$ and CdTe (Fig. 1.6), (b) $E_{\mathrm{g}}$ being close to the optimum value of $1.4 \mathrm{eV}$ (Fig. 1.5 in Vol. 2) and the sharp absorption onset near $E_{\mathrm{g}}$ (low $E_{\mathrm{U}}$ ) (Fig. 16.6), which is expected to reduce the open-circuit voltage loss [see Fig. 1.7(b) in Vol. 2] [45], (c) efficient free carrier generation by non-excitonic semiconductor-type transitions within the $\mathrm{PbI}_{3}{ }^{-}$ 
component in the whole visible region (Fig. 16.9), (d) low levels of carrier recombination by electric-field assisted carrier collection (Figs. 16.17 and 16.18) and (e) the high optical confinement and low parasitic absorption in the solar cells (Figs. 16.16 and 16.19).

\subsection{Real Time Spectroscopic Ellipsometry of Thin Film Perovskites}

Real time spectroscopic ellipsometry (RTSE) monitoring enables fundamental studies of (i) the growth evolution of both perovskite thin films ( $\mathrm{MAPbI}_{3}$ and related materials) and component materials ( $\mathrm{PbI}_{2}$ and other inorganics, MAI and other organics) as well as (ii) the stability of the resultant films post-deposition. These studies enable detection of phase instabilities and changes in composition and structure occurring either during growth as a function of different deposition conditions or after deposition as a function of external stimuli known to cause perovskite decomposition or phase changes.

There are several methods already employed for perovskite deposition, each with their own region of parameter space yielding optimized materials for devices. Some of these methods are more easily adapted to RTSE monitoring of film growth than others. One case study described here will involve vapor deposition of $\mathrm{MAPbI}_{3}$ enabled by evaporation of $\mathrm{PbI}_{2}$ in a MAI vapor rich vacuum chamber. Figure 16.20 shows a schematic of a vacuum chamber outfitted with windows for in situ RTSE measurements during evaporation of $\mathrm{PbI}_{2}, \mathrm{MAI}$, and other source materials. This evaporation-based approach has multiple utility: (i) PV device quality $\mathrm{MAPbI}_{3}$ can be made as films and layers in functioning solar cells and (ii) fundamental studies of the component materials MAI and $\mathrm{PbI}_{2}$ are also enabled. Figure 16.21 shows spectra in $\varepsilon$ for component MAI and $\mathrm{PbI}_{2}$ as obtained from RTSE measurements of their respective component films with analysis using a multiple time point approach to obtain the most average spectra in $\varepsilon$ for each film [106,107]. For single component materials like MAI and $\mathrm{PbI}_{2}$, multiple time and global sum-of- $\sigma(\Sigma \sigma)$ minimization approaches are suitable for ascertaining $\varepsilon$ as well as the time evolution of structural parameters such as bulk layer and surface roughness thickness, as has been detailed for other PV absorber and component materials in other chapters. Spectra in $\varepsilon$ for $\mathrm{MAPbI}_{3}$ shown in Fig. 16.21 has been obtained using a divided spectral range analysis [108] for ellipsometric spectra collected ex situ from a vapor deposited film. This particular sample had relatively high crystalline quality, but a small fraction of $\mathrm{PbI}_{2}$ also present as confirmed with $\mathrm{x}$-ray diffraction and analysis of ellipsometric spectra using $\varepsilon$ for phase-pure solution deposited $\mathrm{MAPbI}_{3}$ and $\mathrm{PbI}_{2}$ in a Bruggeman effective medium approximation [107]. 


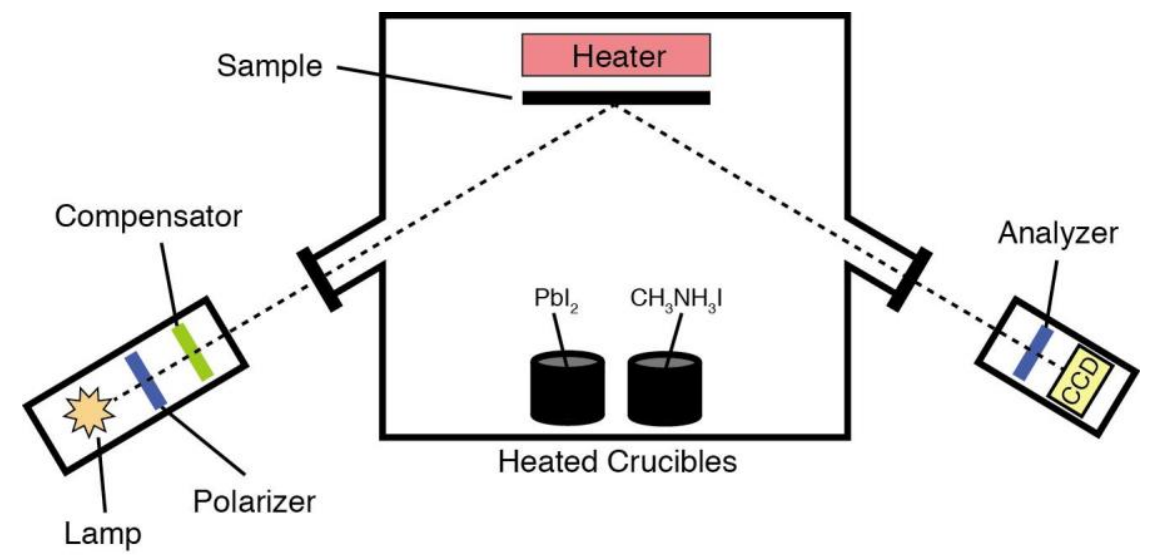

Fig. 16.20 Schematic for real time spectroscopic ellipsometry (RTSE) monitoring of $\mathrm{MAPbI}_{3}$ from multi-source vapor deposition. Adapted from [106].
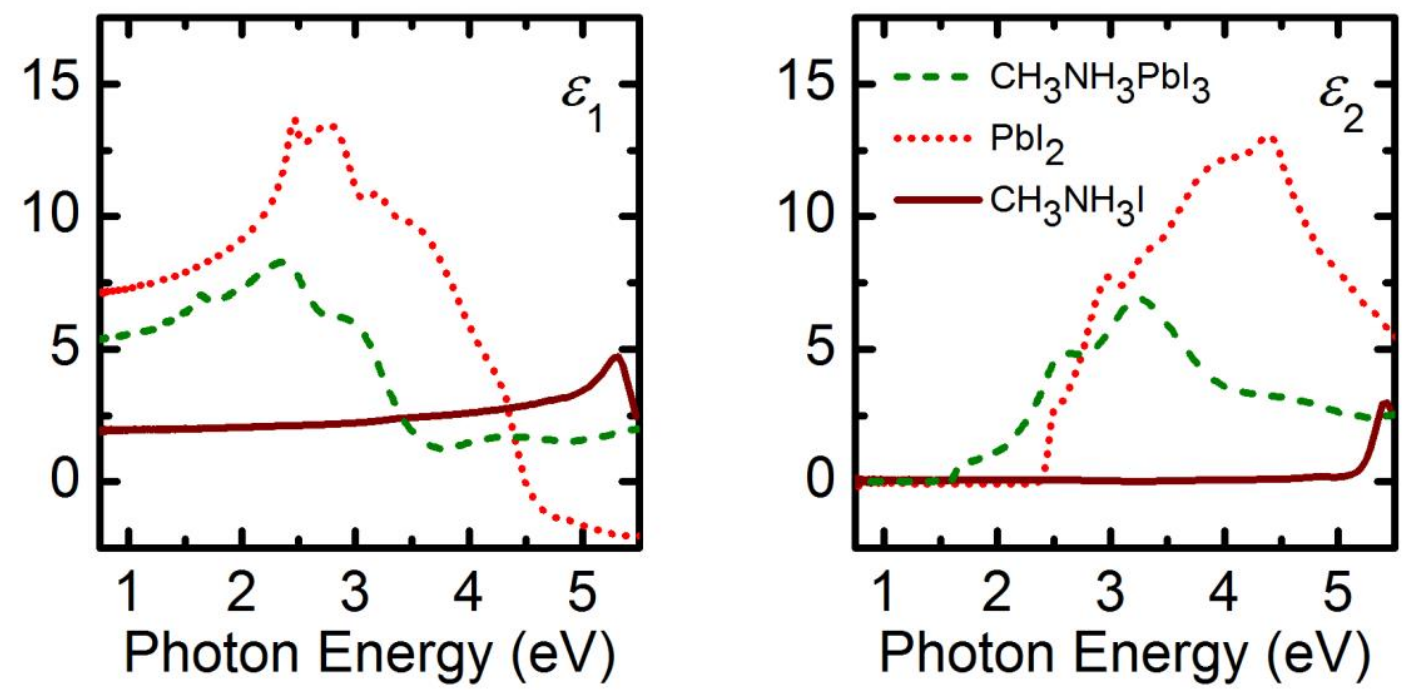

Fig. 16.21 Reference spectra in $\varepsilon$ for vapor deposited $\mathrm{PbI}_{2}, \mathrm{MAI}$, and $\mathrm{MAPbI}_{3}$ used for analysis of RTSE data collected during growth and degradation [107].

Due to the optical contrast between MAI, $\mathrm{PbI}_{2}$, and $\mathrm{MAPbI}_{3}$ shown in Fig. 16.21, it is possible to deduce some relative fractional components of each in a phase-segregated multi-component film from ellipsometric spectra. When ellipsometric spectra are collected in situ as a function of time, like RTSE, it is possible to better understand the dynamics of what occurs-namely the appearance or disappearance of particular 
components. Two case studies to be investigated here involve monitoring (i) vapor deposition of perovskite films enabled by in situ RTSE measurements and (ii) degradation of a film post-deposition upon exposure to laboratory ambient air. For studies of growth evolution, the deposition system must be adaptable to in situ RTSE via appropriate windows and a clear beam-path. In the case of post-deposition changes in films, all that is required is that a sample be first produced and that the post-deposition environment enables in situ ellipsometry measurements. Here we have applied the simplest post-deposition environment possible, namely leaving the sample exposed to laboratory ambient conditions while mounted upon a spectroscopic ellipsometer.

\subsubsection{RTSE during Vapor Deposition}

As the first example, consider RTSE measurements collected in situ during thin film vapor deposition using MAI and $\mathrm{PbI}_{2}$ sources. The intended film composition is predominately polycrystalline $\mathrm{MAPbI}_{3}$, possibly with some other components to manipulate the grain boundary chemistry. If not in the appropriate region of deposition parameter space, it is likely to produce significant amounts of phase-segregated $\mathrm{PbI}_{2}+$ MAI instead of the desired perovskite. These phase segregated regions have been observed near the substrate/film and film/ambient interfaces, possibly where interfacial effects impact perovskite stability. The relative amounts of all components may vary with time during deposition. For vapor deposited materials, changes in source-to-substrate throw distance and deposition sequence (simultaneous or alternating source fluxes) can manipulate the final relative amounts of each component. By analyzing vapor deposited films prepared under a variety of conditions, a relatively flexible structural model has been developed to analyze ellipsometric spectra as shown in Fig. 16.22 [106]. The model itself relies on (i) a known substrate, with optical properties and structure most directly obtained from an ellipsometry measurement prior to film deposition, (ii) a substrate-adjacent Bruggeman effective medium approximation (EMA) layer consisting of $\mathrm{PbI}_{2}+\mathrm{MAI}$, (iii) a $\mathrm{MAPbI}_{3}$ layer, (iv) a EMA layer of $\mathrm{PbI}_{2}+\mathrm{MAPbI}_{3}+$ void, and (v) a surface roughness EMA layer of 0.5 void and $0.5 \mathrm{PbI}_{2}+\mathrm{MAI}$ material fractions. Reference spectra in $\varepsilon$ shown in Fig. 16.21 are used for the component materials in this structure. To aid in interpretation of the results, individual layer thicknesses and material fractions are combined into effective material thicknesses $\left(d_{\text {eff }}\right)$ by multiplying each layer thickness (d) by the relative material fraction within that layer $(f)$ such that 


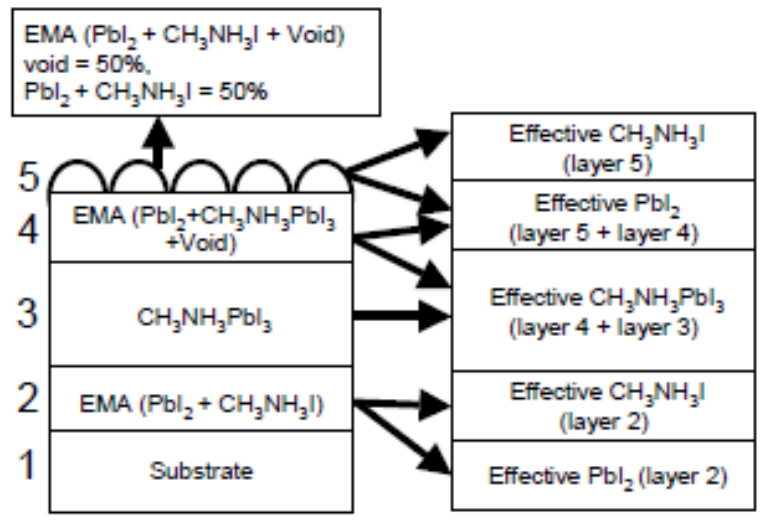

Fig. 16.22 Structure model used in analysis of RTSE data collected during vapor deposition and post-deposition degradation. The layered structure for analysis of ellipsometric spectra consists of (i) a known substrate, (ii) a substrate-adjacent Bruggeman effective medium approximation (EMA) layer consisting of $\mathrm{PbI}_{2}+\mathrm{MAI}$, (iii) a $\mathrm{MAPbI}_{3}$ layer, (iv) a EMA layer of $\mathrm{PbI}_{2}+\mathrm{MAPbI}_{3}+$ void, and (v) a surface roughness EMA layer of 0.5 void and $0.5 \mathrm{PbI}_{2}+\mathrm{MAI}$ material fractions. The effective material thicknesses of $\mathrm{MAPbI}_{3}, \mathrm{PbI}_{2}$ and MAI near the substrate, and surface $\mathrm{PbI}_{2}$ and MAI are calculated from the sum of the layer thicknesses weighted by the respective material fraction. Adapted from [106,107].

$d_{\text {eff }}=d \times f$. These effective material thicknesses of $\mathrm{MAPbI}_{3}, \mathrm{PbI}_{2}$ and MAI near the substrate, and surface $\mathrm{PbI}_{2}$ and MAI are calculated from the sum of effective material thicknesses of each layer.

Figure 16.23 shows effective thicknesses of the phase segregated $\mathrm{PbI}_{2}$ and MAI near the film surface and near the substrate as well as the $\mathrm{MAPbI}_{3}$ component [106]. This particular sample has been made on thermal oxide ( $250 \AA$ thick) coated crystal silicon with a throw distance of $50 \mathrm{~cm}$ and MAI and $\mathrm{PbI}_{2}$ sources heated to 100 and $300^{\circ} \mathrm{C}$, respectively. For the first $\sim 10$ minutes of the deposition, $\mathrm{MAPbI}_{3}$ grows with some excess surface $\mathrm{PbI}_{2}$. $\mathrm{MAPbI}_{3}$ thickness increases throughout the deposition, however at 10 minutes of growth effective thicknesses of $\mathrm{PbI}_{2}$ and MAI near the substrate begin to increase. After 20 minutes variable amounts of both $\mathrm{PbI}_{2}$ and MAI appear on the film surface. The appearance of significant amounts of both $\mathrm{PbI}_{2}$ and MAI near the surface and substrate interfaces implies that moderately thin $\mathrm{MAPbI}_{3}$ grown under these conditions may not be stable. After sufficient time has passed, $\sim 72$ minutes, the 

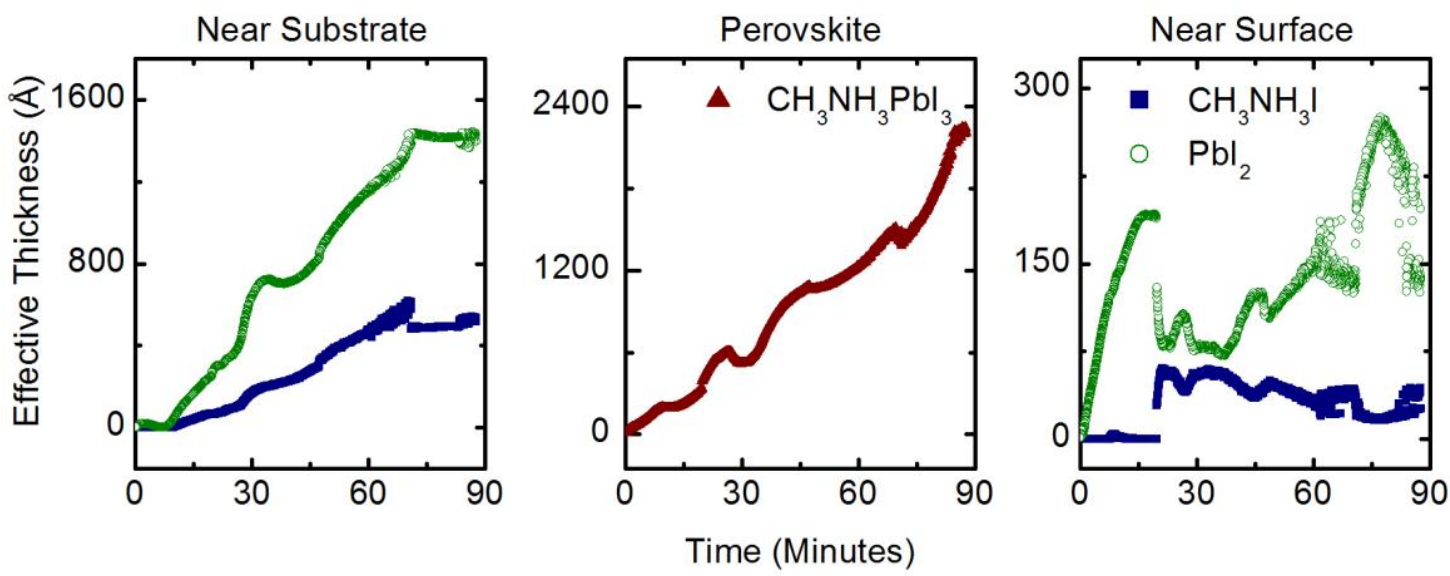

Fig. 16.23 Growth evolution from RTSE in the form of effective material thicknesses of $\mathrm{MAPbI}_{3}, \mathrm{PbI}_{2}$ and MAI near the substrate, and surface $\mathrm{PbI}_{2}$ and MAI as functions of time during vapor deposition [106].

near-substrate $\mathrm{PbI}_{2}$ and MAI effective thicknesses stabilize and the $\mathrm{MAPbI}_{3}$ thickness deposition rate increases.

To summarize, RTSE analysis of this deposition shows three stages: (i) an initial thin $\mathrm{MAPbI}_{3}$ layer with a slightly lower growth rate from 0-10 minutes, (ii) continued $\mathrm{MAPbI}_{3}$ growth accompanied by phase segregation of MAI and $\mathrm{PbI}_{2}$ from 10-72 minutes, and (iii) stabilization of MAI and $\mathrm{PbI}_{2}$ effective thicknesses and an increase in growth rate for $\mathrm{MAPbI}_{3}$ from 72 minutes until the end of the deposition. Samples prepared under different deposition conditions have shown immediate appearance of MAI and $\mathrm{PbI}_{2}$ components with the perovskite, appearance of these components followed by formation of the perovskite, or different behaviors for each component layer. The ability to apply RTSE during vapor deposition shows the possibility of real time monitoring and control of film composition. These types of RTSE measurements have been made both on test substrates for underlying smoothness and structural simplicity (e.g. oxide covered crystal silicon and glass) and in the superstrate PV device configuration on rough $\mathrm{SnO}_{2}: \mathrm{F}$ coated glass with an electron selective layer (ESL). 


\subsubsection{RTSE Post-Deposition}

As mentioned elsewhere in this chapter, these perovskites may undergo reversible or irreversible changes with temperature variations, applied bias, exposure to moisture and humidity, and under ultraviolet light. In principle, in situ spectroscopic ellipsometry enables monitoring of the changes in optical response and structure for samples prepared by any deposition method if the measurements can be conducted in a suitably controlled environment so that "before" and "after" characteristics are observed. In situ RTSE enables data to be collected during the respective process, whatever it may be. Temperature controlled stages, biased electrical contacts on films, environmental chambers, and unpolarized intense light sources can be readily applied to these kinds of samples. The sample may begin to change immediately upon exposure to laboratory ambient conditions. This exposure to laboratory ambient, nominally room temperature $\sim 20^{\circ} \mathrm{C}$, illuminated, and relative humidity $\sim 50 \%$, may cause a $\mathrm{MAPbI}_{3}$ perovskite to change and decompose into its constituent components $\mathrm{PbI}_{2}$ and MAI.

In situ RTSE has been performed on a vapor deposited initially predominately $\mathrm{MAPbI}_{3}$ film prepared on soda lime glass placed on an ellipsometer [107]. Ellipsometric spectra have been collected every 5 minutes for 48 hours. To fit ellipsometric spectra at each measurement time, reference $\varepsilon$ from Fig. 16.21 and the structural model from Fig. 16.22 used to describe RTSE of vapor deposition growth have been applied to describe phase segregation and film decomposition. The behavior of the sample post-deposition in ambient laboratory conditions shows similarities to that observed for un-optimized material during growth. Namely, phase segregated $\mathrm{MAI}$ and $\mathrm{PbI}_{2}$ components accumulate near the substrate/film and film/ambient interfaces. Effective material component thicknesses are shown in Fig. 16.24. It is clear that the $\mathrm{MAPbI}_{3}$ effective thickness decreases with time over the RTSE measurements. This decrease in effective $\mathrm{MAPbI}_{3}$ thickness occurs with initial increases in the MAI and $\mathrm{PbI}_{2}$ effective thicknesses near the substrate and surface, implying that phase segregation of the perovskite has occurred. After about 1500-2000 minutes have passed, the amount of MAI and $\mathrm{PbI}_{2}$ components near the substrate begin to decrease while the $\mathrm{PbI}_{2}$ near the surface continues to increase and the surface MAI thickness is much lower in magnitude. The sum of all $\mathrm{PbI}_{2}$ increases with time, $\mathrm{MAPbI}_{3}$ decreases with time, and MAI first increases then weakly decreases. This indicates that as some of the perovskite is decomposing, the constituents may be reacting. 

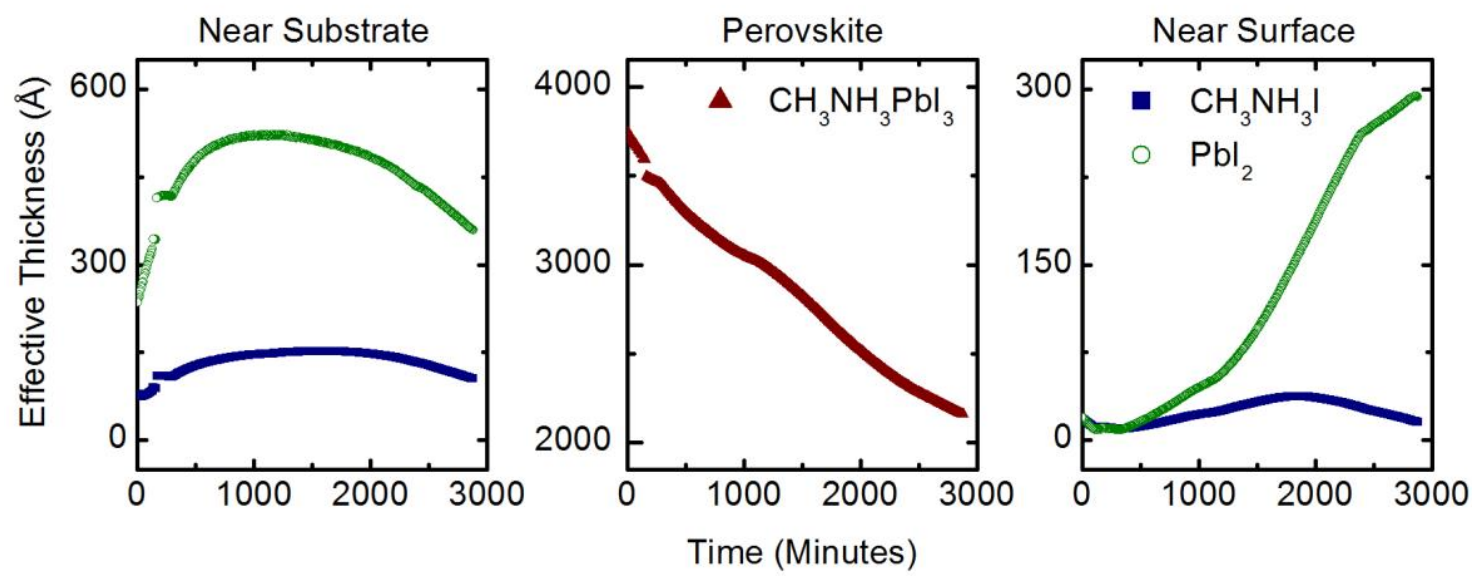

Fig. 16.24 Effective material thicknesses of $\mathrm{MAPbI}_{3}, \mathrm{PbI}_{2}$ and MAI near the substrate, and surface $\mathrm{PbI}_{2}$ and MAI as functions of time during post-deposition exposure in laboratory ambient conditions monitored by RTSE [107].

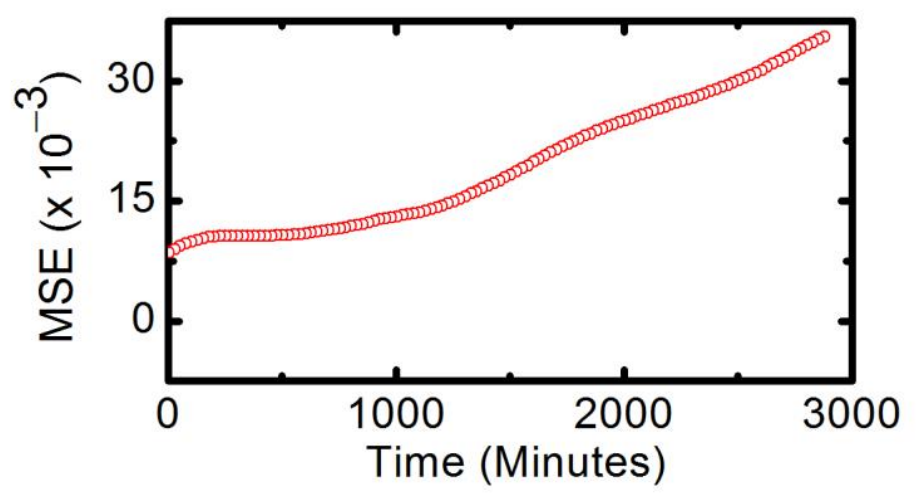

Fig. 16.25 Mean square error (MSE) as a function of time for fits to RTSE data collected during post-deposition exposure to laboratory ambient conditions, with results in Fig. 16.24 [109].

An interesting observation comes from the quality of the fit over the course of decomposition, represented by the mean square error (MSE) in Fig. 16.25. Reference spectra in $\varepsilon$ are used over the full measurement time, however the quality of the fit decreases (MSE increases) at later times. Recent work has shown that changes in the perovskite component optical properties can reduce and stabilize the MSE [109]. A possible explanation for the changes in $\varepsilon$ observed may be related to the sum of the effective MAI thicknesses. When considering the density and expected amount of MAI and $\mathrm{PbI}_{2}$ produced by decomposition of $\mathrm{MAPbI}_{3}$, the effective thicknesses shown 
in Fig. 16.24 imply that some MAI is not accounted in the model. Process parameter dependent phase diagrams for these perovskites [110] indicate that a "stacked perovskite sheet" structure consisting of thin perovskite layers interspaced by MAI exists. This explanation possibly accounts for the missing effective MAI thickness, and the transition from perovskite to stacked perovskite sheet structure could be responsible for the variation in $\varepsilon$ needed to reduce MSE of RTSE data fitting at later times during degradation.

\subsection{Characterization of Degradation in Hybrid Perovskite Materials}

One of the key aspects to achieve the full potential of PV devices based on hybrid perovskites is to control the stability of these materials. Up to date, different fabrication protocols can be followed resulting in different film morphologies which might lead to variable degradation pathways [111]. However, most approaches to produce materials, mainly low-temperature evaporation or solution-based, are apt to result in similar high-performance materials and subsequent devices with rather comparable efficiencies and lifetimes. Several environmental factors such as humidity, heat, and illumination can compromise the stability both of the perovskite materials, in particular $\mathrm{MAPbI}_{3}$, and of the solar cell devices. The effect of humidity as the main degradation agent has been investigated by SE, and different results have been detected, from reversible hydration to irreversible decomposition of $\mathrm{MAPbI}_{3}$ [51]. The perovskite structure of $\mathrm{MAPbI}_{3}$ is depicted in Fig. 16.26(a). Two hydrated $\mathrm{MAPbI}_{3}$ crystal phases form by water incorporation with either one or two water molecules per formula unit. The monohydrate and dihydrate crystal structures are shown in Fig. 16.26 (b) and (c), respectively.
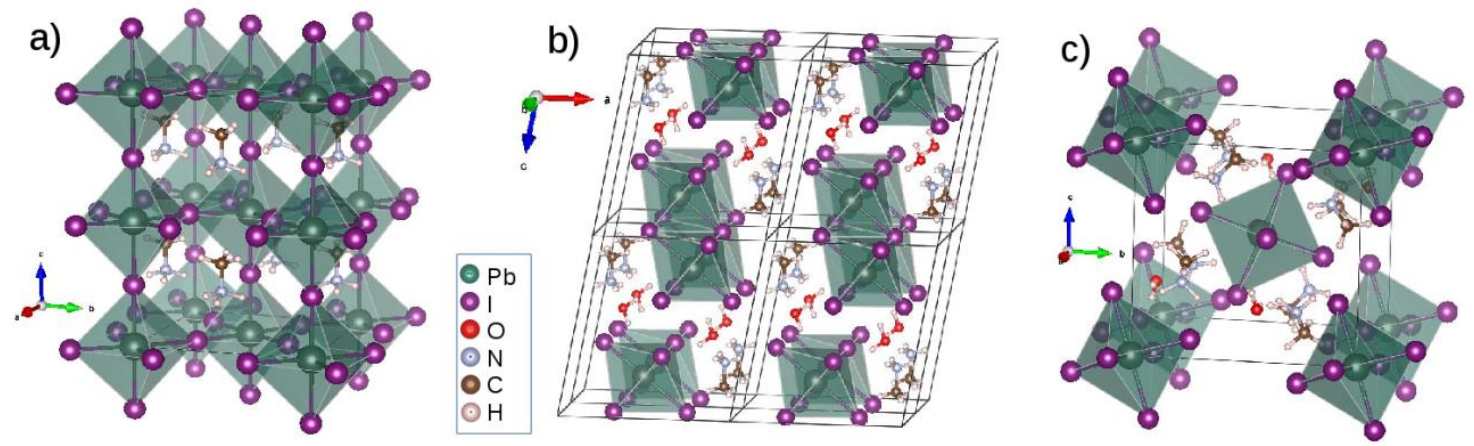

Fig. 16.26 Crystal structures of the phases related to hydration of $\mathrm{MAPbI}_{3}$ : (a) pristine perovskite phase, (b) $\mathrm{MAPbI}_{3} \bullet \mathrm{H}_{2} \mathrm{O}$ (monohydrate), and (c) $\mathrm{MAPbI}_{3} \cdot 2 \mathrm{H}_{2} \mathrm{O}$ (dihydrate). 


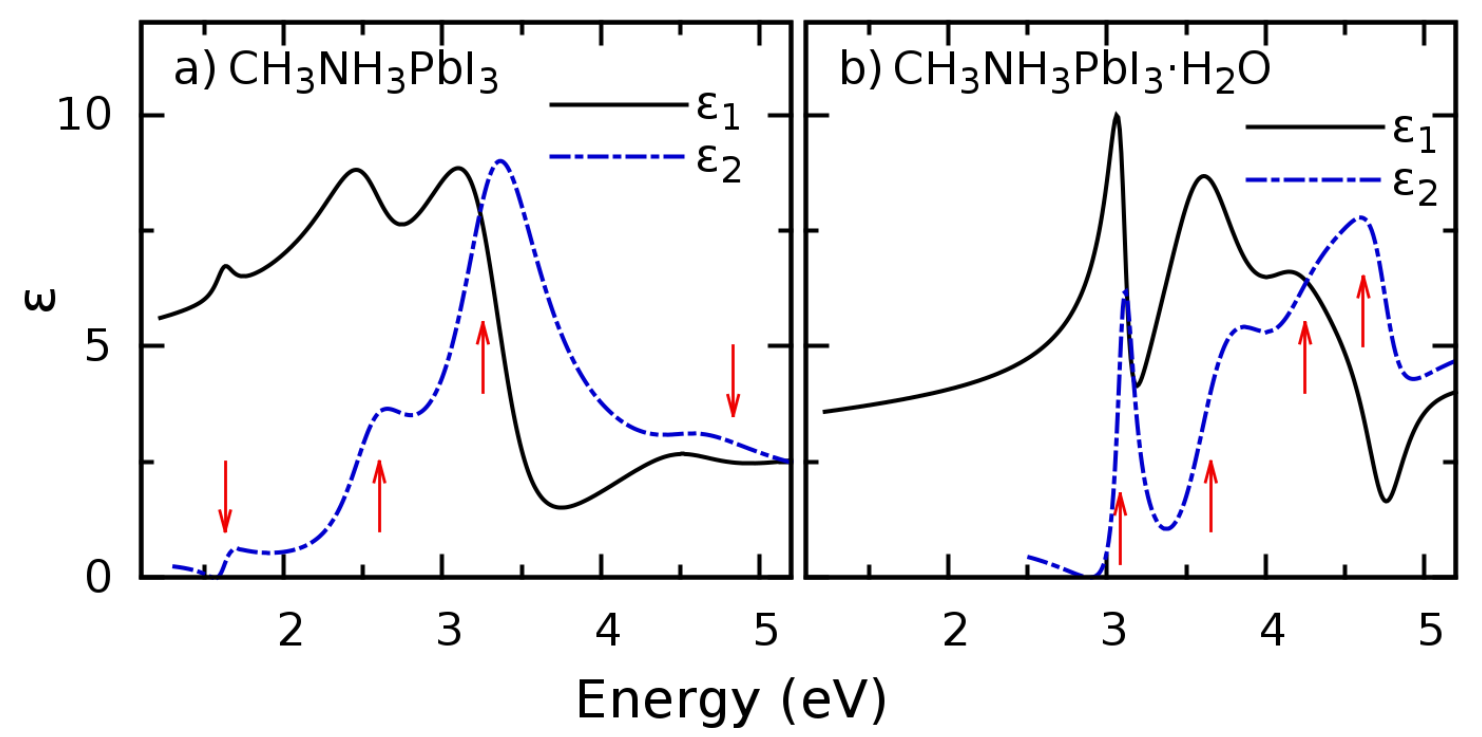

Fig. 16.27 Dielectric functions determined by ellipsometry of a) $\mathrm{MAPbI}_{3}$ single crystal and b) $\mathrm{MAPbI}_{3} \cdot \mathrm{H}_{2} \mathrm{O}$ monohydrate phase. Data from Ref. [51]. Values of $\varepsilon_{2}$ in the sub-bandgap region are spurious, due to the modelling. Arrows indicate the energies of the main critical points obtained from fits to second derivative spectra.[51,52]

In the seminal work by Leguy et al. [51], solution-processed perovskite films, single crystals and full devices were exposed to high relative humidity $(\mathrm{RH})$ around $75 \%$ $( \pm 5 \%)$. The hydration mechanism was first investigated optically by SE on single crystals of $\mathrm{MAPbI}_{3}$. The crystals were exposed to a humid ambient with nitrogen as carrier gas at room temperature $\left(21^{\circ} \mathrm{C}\right)$. Figure 16.27 shows the dielectric function determined in a crystal before (a) and after $60 \mathrm{~h}$ of exposure (b) to the humid ambient. The effect of water was to form a new crystal structure identified by $x$-ray diffraction as the monohydrate $\mathrm{MAPbI}_{3} \cdot \mathrm{H}_{2} \mathrm{O}$, see Fig. 16.26(b). The initially black $\mathrm{MAPbI}_{3}$ crystal with band gap $\sim 1.6 \mathrm{eV}$ becomes transparent (whitish) in the wet ambient, with a band gap of $\sim 3.1 \mathrm{eV}$.

When subsequently exposed to a dry atmosphere, the crystal instantly starts to convert back to the initial structure, as displayed in Fig. 16.28, with the process ending in the full recovery of the material. The spectra measured both during hydration or dehydration can be well understood by an effective Bruggeman mixture of the two phases, therefore suggesting that water is highly mobile within the perovskite lattice with the transformation occurring more or less homogeneously throughout the sample. Both from diffraction and SE investigations it appears that dehydration is faster than 
hydration. However, the time needed for the transformation depends from the initial material condition: the presence of defects such as pores or grain boundaries facilitate water diffusion through the sample. Consequently, the transformation of thin polycrystalline films starts almost instantaneously [51,59] because water penetrates more efficiently within the grains than in compact single crystals. The study on thin films [51] lead to the following equations for the hydration of $\mathrm{MAPbI}_{3}$ :

$4\left(\mathrm{CH}_{3} \mathrm{NH}_{3}\right) \mathrm{PbI}_{3}+4 \mathrm{H}_{2} \mathrm{O} \leftrightarrow 4\left[\mathrm{CH}_{3} \mathrm{NH}_{3} \mathrm{PbI}_{3} \cdot \mathrm{H}_{2} \mathrm{O}\right]$

$\leftrightarrow\left(\mathrm{CH}_{3} \mathrm{NH}_{3}\right)_{4} \mathrm{PbI}_{6} \cdot 2 \mathrm{H}_{2} \mathrm{O}+3 \mathrm{PbI}_{2}+2 \mathrm{H}_{2} \mathrm{O}$

Eq. (16.2) describes a two-step process in which the crystal structure of $\mathrm{MAPbI}_{3}$ is first saturated with one water molecule per unit cell, followed by the insertion of a second water molecule upon longer exposure to humidity. Accordingly, the monohydrate is an intermediate product that can be easily converted back to $\mathrm{MAPbI}_{3}$. However, when the reaction is driven further to the right of the stoichiometric equation (16.2), the formation of the dihydrated crystal phase initiates the decomposition with formation of $\mathrm{PbI}_{2}$ and release of water ending in an irreversible degradation of the film. In polycrystalline film material the transformation is reversible up to a certain point before any dihydrate formation. Later, both reactions may happen simultaneously at different film locations. For instance, decomposition may appear at grain boundaries while there are still grains of pristine $\mathrm{MAPbI}_{3}$. In Ref. [51], this situation was detected after a monohydrate content in the film of about $20 \%$ was reached. Then, the roughness increased dramatically with formation of needle-like structures which are presumably the dihydrate. Therefore, the exact degradation pathways depend on the sample morphology. Furthermore, temperature and illumination also play a role but the interplay of all causes is not well established yet. A decisive factor is whether water condensation occurs on the surface of the film or not [51,111]. If water condensation is avoided, the process is reversible in films and even in solar cells, where the photovoltaic performance of "degraded" devices was recovered with a simple drying step under a nitrogen stream for a few hours [51]. 


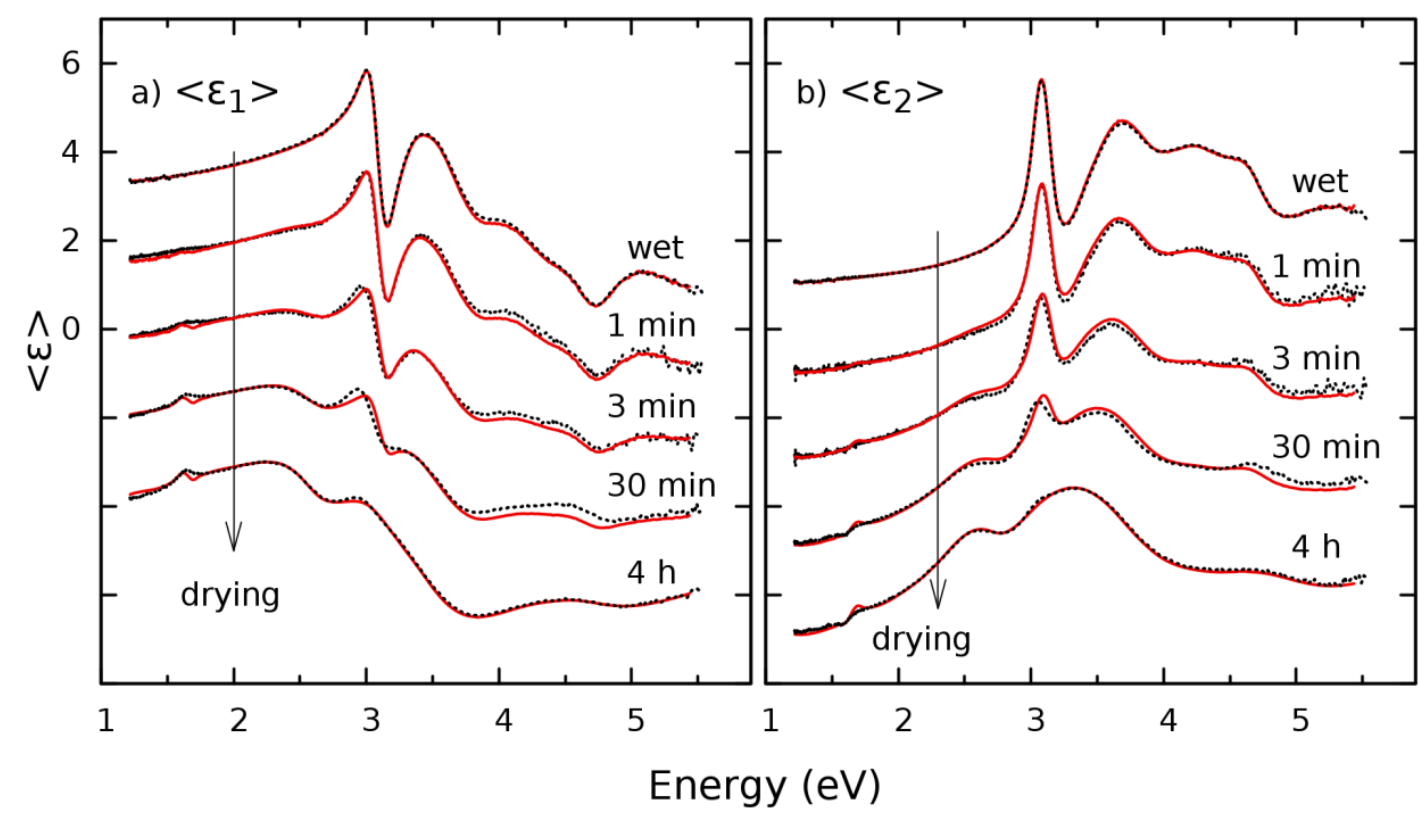

Fig. 16.28 Ellipsometric spectra presented as pseudo-dielectric functions measured during the back-conversion from a $\mathrm{MAPbI}_{3} \cdot \mathrm{H}_{2} \mathrm{O}$ monohydrate crystal to the pristine $\mathrm{MAPbI}_{3}$ phase, see Ref. [51]. Dotted lines display the experimental results and solid lines the simulations based on an effective Bruggeman mixture of the two phases and voids. The chosen time intervals represent approximately equidistant volume fractions, i.e., variations of 0.25 in hydrate content upon drying from 1 (wet) to 0 . The vertical scale corresponds to the uppermost spectrum and the rest are shifted downwards by steps of -2 for clarity.

Shirayama et al. [59] have characterized the degradation processes of ultra-smooth MAPI thin films prepared by a laser evaporation technique. In this study, the exposure of the $\mathrm{MAPbI}_{3}$ samples to humid air was carried out under ordinary room illumination. They observed that at $75 \% \mathrm{RH}$, the surface reaction was quite rapid and the mirror-like surface of the pristine $\mathrm{MAPbI}_{3}$ layer became whitish within $20 \mathrm{~s}$ after the air exposure. Unfortunately, reliable optical characterization of this process was quite difficult due to the extensive developed roughness and further analysis was not made. The authors monitored the film changes at RH of $40 \%$, observing by SEM that even at this relatively low RH value [112], large structural changes in the near-surface region take place after a short exposure. The changes in optical functions are displayed in Fig. 16.29 as pseudo-dielectric imaginary part $\left\langle\varepsilon_{2}\right\rangle$. Although SEM 


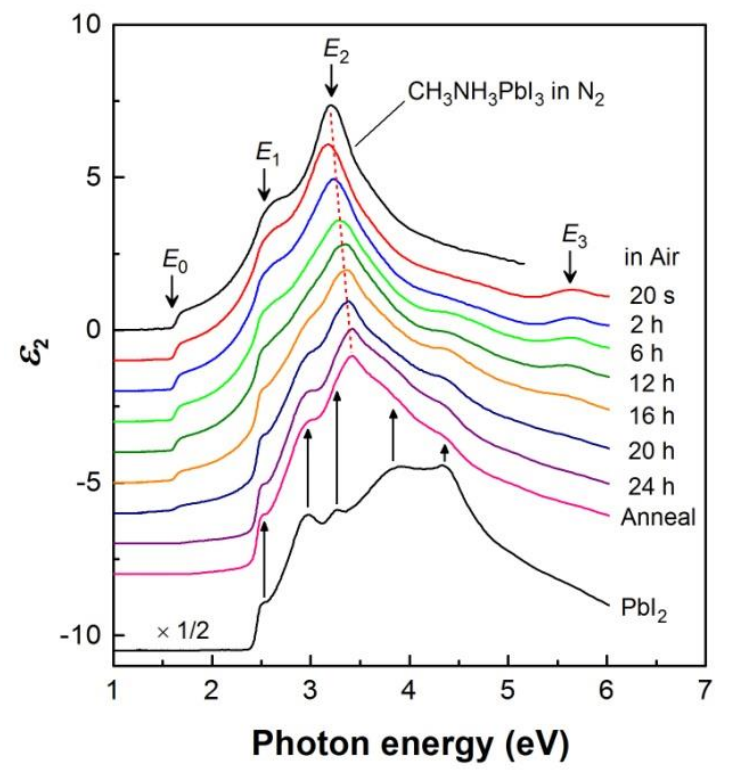

Fig. 16.29 Variation of $\varepsilon_{2}$ reported in Ref. [59] for a $\mathrm{MAPbI}_{3}$ thin film during exposure to air at $40 \% \mathrm{RH}$. The spectra of $\mathrm{MAPbI}_{3}$ in $\mathrm{N}_{2}$ and of a $\mathrm{PbI}_{2}$ film are plotted for reference. The degraded sample spectrum does not change after a thermal annealing at $100^{\circ} \mathrm{C}$, indicating its stability.

indicated a much faster surface transformation, changes in the optical properties start to be evident at times beyond $2 \mathrm{~h}$. In particular, absorption features that coincide with those of a $\mathrm{PbI}_{2}$ reference start to appear clearly while the band gap transition at $1.6 \mathrm{eV}$ gradually disappears with the exposure time until at $\mathrm{t} \geq 24 \mathrm{~h}$, the bandgap is located at $2.4 \mathrm{eV}$, consistent with that of $\mathrm{PbI}_{2}$. Even if during the initial degradation process $(\mathrm{t} \leq 3$ h) the change of the $\left\langle\varepsilon_{2}\right\rangle$ spectrum is rather small, the $E_{2}$ peak shows a distinct peak shift which has been related to the formation of the hydrate phase in the surface region. In contrast to the film thickness increase during hydration observed in Ref. [51], at low $\mathrm{RH}$ [59] a reduction of the total layer thickness was observed. This was explained by the concurrence of a desorption reaction in addition to the hydration reactions of Eq. (16.2). The thickness reduction occurs according to:

$$
\mathrm{CH}_{3} \mathrm{NH}_{3} \mathrm{PbI}_{3} \rightarrow \mathrm{PbI}_{2}+\mathrm{CH}_{3} \mathrm{NH}_{3} \mathrm{I}\left(\text { or } \mathrm{CH}_{3} \mathrm{NH}_{2}+\mathrm{HI}\right)
$$

In this study it was emphasized that the time scale of the $\mathrm{MAPbI}_{3}$ degradation observed at $40 \%$ RH (1 day) starts from the surface and is much faster than that reported in earlier studies at similar RH (1 month) without illumination [113]. This correlates quite well with the efficiency loss observed in actual solar cells (1-2 days at 55\% RH) 
[54]. Accordingly, the degradation of hybrid perovskite solar cells in humid air is most likely caused by the rapid changes near the interfaces that conform the devices. In fact, the contacts performance has been found to degrade faster than the perovskite active layer itself [114].

As a conclusion of these studies, it seems that the degradation processes depend strongly both on the morphology of the samples and on the ambiental conditions, but mainly on the latter. Although humidity is an essential parameter, also temperature and illumination are important degradation agents. Structural studies combined with SE measurements provide insight into the degradation pathways. SE monitoring can be very helpful to follow the changes, allowing to identify the degradation species and to keep track of the thickness and roughness variations associated to these changes.

\section{Acknowledgements}

N. J. Podraza and K. Ghimire would like to acknowledge D. Zhao, A. Cimaroli, Y. Ke, F. Hong, T. Shi, and Prof. Y. Yan for providing samples and materials; M. Junda for graphical assistance; and the National Science Foundation for financial support (CHE-1230246). M. I. Alonso acknowledges financial support from the Spanish Ministry of Economy and Competitiveness, through grants CSD2010-00044, MAT2015-70850-P, and the "Severo Ochoa" Programme for Centres of Excellence in R\&D (SEV-2015-0496). 


\section{References}

1. W. S. Yang, J. H. Noh, N. J. Jeon, Y. C. Kim, S. Ryu, J. Seo, S. I. Seok, Science 348, $1234(2015)$

2. D. Bi, W. Tress, M. I. Dar, P. Gao, J. Luo, C. Renevier, K. Schenk, A. Abate, F. Giordano, J.-P. Correa Baena, J.-D. Decoppet, S. M. Zakeeruddin, M. K. Nazeeruddin, M. Grätzel, A. Hagfeldt, Sci. Adv. 2, e1501170 (2016)

3. M. Saliba, T. Matsui, J.-Y. Seo, K. Domanski, J.-P. Correa-Baena, M. K. Nazeeruddin, S. M. Zakeeruddin, W. Tress, A. Abate, A. Hagfeldt, M. Grätzel, Energy Environ. Sci. 9, 1989 (2016)

4. T. J. Jacobsson, J.-P. Correa-Baena, M. Pazoki, M. Saliba, K. Schenk, M. Grätzel, A. Hagfeldt, Energy Environ. Sci. 9, 1706 (2016)

5. A. Kojima, K. Teshima, Y. Shirai, T. Miyasaka, J. Am. Chem. Soc. 131, 6050 (2009)

6. M. M. Lee, J. Teuscher, T. Miyasaka, T. N. Murakami, H. J. Snaith, Science 338, 643 (2012)

7. M. Liu, M. B. Johnston, H. J. Snaith, Nature 501, 395 (2013)

8. J. Burschka, N. Pellet, S.-J. Moon, R. Humphry-Baker, P. Gao, M. K. Nazeeruddin, M. Grätzel, Nature 499, 316 (2013)

9. T. Baikie, Y. Fang, J. M. Kadro, M. Schreyer, F. Wei, S. G. Mhaisalkar, M. Grätzel, T. J. White, J. Mater. Chem. A 1, 5628 (2013)

10. P. Gao, M. Grätzel, M. K. Nazeeruddin, Energy Environ. Sci. 7, 2448 (2014)

11. M. A. Green, A. Ho-Baillie, H. J. Snaith, Nat. Photon. 8, 506 (2014)

12. H. S. Jung, N. -G. Park, Small 11, 10 (2015)

13. T. M. Brenner, D. A. Egger, L. Kronik, G. Hodes, D. Cahen, Nat. Rev. Mater. 1, 15007 (2016)

14. J. S. Manser, J. A. Christians, P. V. Kamat, Chem. Rev. 116, 12956 (2016)

15. T. M. Koh, K. Fu, Y. Fang, S. Chen, T. C. Sum, N. Mathews, S. G. Mhaisalkar, P. P. Boix, T. Baikie, J. Phys. Chem. C 118, 16458 (2014)

16. G. E. Eperon, S. D. Stranks, C. Menelaou, M. B. Johnston, L. M. Herz, H. J. Snaith, Energy Environ. Sci. 7, 982 (2014)

17. S. Pang, H. Hu, J. Zhang, S. Lv, Y. Yu, F. Wei, T. Qin, H. Xu, Z. Liu, G. Cui, Chem. Mater. 26, 1485 (2014)

18. Q. Han, S.-H. Bae, P. Sun, Y.-T. Hsieh, Y. Yang, Y. S. Rim, H. Zhao, Q. Chen, W. Shi, G. Li, Y. Yang, Adv. Mater. 28, 2253 (2016)

19. M. T. Weller, O. J. Weber, J. M. Frost, A. Walsh, J. Phys. Chem. Lett. 6, 3209 (2015) 
20. N. Pellet, P. Gao, G. Gregori, T.-Y. Yang, M. K. Nazeeruddin, J. Maier, M. Grätzel, Angew. Chem. Int. Ed. 53, 3151 (2014)

21. J.-W. Lee, D.-H. Kim, H.-S. Kim, S.-W. Seo, S. M. Cho, N.-G. Park, Adv. Energy Mater. 5, 1501310 (2015)

22. Z. Li, M. Yang, J.-S. Park, S.-H. Wei, J. J. Berry, K. Zhu, Chem. Mater. 28, 284 (2016)

23. C. Yi, J. Luo, S. Meloni, A. Boziki, N. Ashari-Astani, C. Grätzel, S. M. Zakeeruddin, U. Röthlisberger, M. Grätzel, Energy Environ. Sci. 9, 656 (2016)

24. D. P. McMeekin, G. Sadoughi, W. Rehman, G. E. Eperon, M. Saliba, M. T. Hörantner, A. Haghighirad, N. Sakai, L. Korte, B. Rech, M. B. Johnston, L. M. Herz, H. J. Snaith, Science 351, 151 (2016)

25. J. P. Perdew, K. Burke, M. Ernzerhof, Phys. Rev. Lett. 77, 3865 (1996)

26. M. Shirayama, H. Kadowaki, T. Miyadera, T. Sugita, M. Tamakoshi, M. Kato, T. Fujiseki, D. Murata, S. Hara, T. N. Murakami, S. Fujimoto, M. Chikamatsu, H. Fujiwara, Phys. Rev. Applied 5, 014012 (2016)

27. M. Kato, T. Fujiseki, T. Miyadera, T. Sugita, S. Fujimoto, M. Tamakoshi, M. Chikamatsu, H. Fujiwara, J. Appl. Phys. 121, 115501 (2017)

28. D. Shi, V. Adinolfi, R. Comin, M. Yuan, E. Alarousu, A. Buin, Y. Chen, S. Hoogland, A. Rothenberger, K. Katsiev, Y. Losovyj, X. Zhang, P. A. Dowben, O. F. Mohammed, E. H. Sargent, O. M. Bakr, Science 347, 519 (2015)

29. C. C. Stoumpos, C. D. Malliakas, M. G. Kanatzidis, Inorg. Chem. 52, 9019 (2013)

30. A. Amat, E. Mosconi, E. Ronca, C. Quarti, P. Umari, M. K. Nazeeruddin, M. Grätzel, F. De. Angelis, Nano Lett. 14, 3608 (2014)

31. J.-H. Lee, N. C. Bristowe, P. D. Bristowe, A. K. Cheetham, Chem. Commun. 51, $6434(2015)$

32. J. H. Lee, J.-H. Lee, E.-H. Kong, H. M. Jang, Sci. Rep. 6, 21687 (2016)

33. J. S. Bechtel, R. Seshadri, A. Van der Ven, J. Phys. Chem. C 120, 12403 (2016)

34. R. E. Wasylishen, O. Knop, J. B. Macdonald, Solid State Commun. 56, 581 (1985)

35. E. Mosconi, C. Quarti, T. Ivanovska, G. Ruani, F. De Angelis, Phys. Chem. Chem. Phys. 16, 16137 (2014)

36. A. Mattoni, A. Filippetti, M. I. Saba, P. Delugas, J. Phys. Chem. C 119, 17421 (2015)

37. A. M. A. Leguy, J. M. Frost, A. P. McMahon, V. G. Sakai, W. Kochelmann, C. Law, X. Li, F. Foglia, A. Walsh, B. C. O’Regan, J. Nelson, J. T. Cabral, R. F. Barnes, Nat. Commun. 6, 7124 (2015)

38. M. A. Carignano, A. Kachmar, J. Phys. Chem. C. 119, 8991 (2015) 
39. A. A. Bakulin, O. Selig, H. J. Bakker, Y. L. A. Rezus, C. Müller, T. Glaser, R. Lovrincic, Z. Sun, Z. Chen, A. Walsh, J. M. Frost, T. L. C. Jansen, J. Phys. Chem. Lett. 6, 3663 (2015)

40. M. A. Carignano, Y. Saeed, S. A. Aravindh, I. S. Roqan, J. Even, C. Katan, Phys. Chem. Chem. Phys. 18, 27109 (2016)

41. M. R. Filip, G. E. Eperon, H. J. Snaith, F. Giustino, Nat. Commun. 5, 5757 (2014)

42. J. Kim, S.-C. Lee, S.-H. Lee, K.-H. Hong, J. Phys. Chem. C 119, 4627 (2015)

43. G. Xing, N. Mathews, S. Sun, S. S. Lim, Y. M. Lam, M. Grätzel, S. Mhaisalkar, T. C. Sum, Science 342, 344 (2013)

44. S. Sun, T. Salim, N. Mathews, M. Duchamp, C. Boothroyd, G. Xing, T. C. Sum, Y. M. Lam, Energy Environ. Sci. 7, 399 (2014)

45. S. De Wolf, J. Holovsky, S.-J. Moon, P. Löper, B. Niesen, M. Ledinsky, F.-J. Haug, J.-H. Yum, C. Ballif, J. Phys. Chem. Lett. 5, 1035 (2014)

46. G. Xing, N. Mathews, S. S. Lim, N. Yantara, X. Liu, S. Dharani, M. Grätzel, S. Mhaisalkar, T. C. Sum, Nat. Mater. 13, 476 (2014)

47. Q. Lin, A. Armin, R. C. R. Nagiri, P. L. Burn, P. Meredith, Nat. Photon. 9, 106 (2015)

48. J. M. Ball, S. D. Stranks, M. T. Hörantner, S. Hüttner, W. Zhang, E. J. W. Crossland, I. Ramirez, M. Riede, M. B. Johnston, R. H. Friend, H. J. Snaith, Energy Environ. Sci. 8, 602 (2015)

49. P. Löper, M. Stuckelberger, B. Niesen, J. Werner, M. Filipič, S.-J. Moon, J.-H. Yum, M. Topič, S. De Wolf, C. Ballif, J. Phys. Chem. Lett. 6, 66 (2015)

50. Y. Jiang, M. A. Green, R. Sheng, A. Ho-Baillie, Sol. Eng. Mater. Sol. Cells 137, 253 (2015)

51. A. M. A. Leguy, Y. Hu, M. Campoy-Quiles, M. I. Alonso, O. J. Weber, P. Azarhoosh, M. van Schilfgaarde, M. T. Weller, T. Bein, J. Nelson, P. Docampo, P. R. F. Barnes, Chem. Mater. 27, 3397 (2015)

52. A. M. A. Leguy, P. Azarhoosh, M. I. Alonso, M. Campoy-Quiles, O. J. Weber, J. Yao, D. Bryant, M. T. Weller, J. Nelson, A. Walsh, M. van Schilfgaarde, P. R. F. Barnes, Nanoscale 8, 6317 (2016)

53. T. Miyadera, T. Sugita, H. Tampo, K. Matsubara, M. Chikamatsu, ACS Appl. Mater. Interfaces. 8, 26013 (2016)

54. J. H. Noh, S. H. Im, J. H. Heo, T. N. Mandal, S. I. Seok, Nano Lett. 13, 1764 (2013)

55. G. Niu, X. Guo, L. Wang, J. Mater. Chem. A 3, 8970 (2015)

56. T. Leijtens, G. E. Eperon, N. K. Noel, S. N. Habisreutinger, A. Petrozza, H. J. Snaith, Adv. Energy Mater. 5, 1500963 (2015) 
57. G. Murugadoss, S. Tanaka, G. Mizuta, S. Kanaya, H. Nishino, T. Umeyama, H. Imahori, S. Ito, Jpn. J. Appl. Phys. 54, 08KF08 (2015)

58. T. A. Berhe, W.-N. Su, C.-H. Chen, C.-J. Pan, J.-H. Cheng, H.-M. Chen, M.-C. Tsai, L.-Y. Chen, A. A. Dubale, B.-J. Hwang, Energy Environ. Sci. 9, 323 (2016)

59. M. Shirayama, M. Kato, T. Miyadera, T. Sugita, T. Fujiseki, S. Hara, H. Kadowaki, D. Murata, M. Chikamatsu, H. Fujiwara, J. Appl. Phys. 119, 115501 (2016)

60. H. Fujiwara, J. Koh, P. I. Rovira, R. W. Collins, Phys. Rev. B 61, 10832 (2000)

61. H. Fujiwara, Spectroscopic Ellipsometry: Principles and Applications (Wiley, West Sussex, 2007)

62. N. Kitazawa, Y. Watanabe, Y. Nakamura, J. Mater. Sci. 37, 3585 (2002)

63. W.-J. Yin, T. Shi, Y. Yan, Adv. Mater. 26, 4653 (2014)

64. W.-J. Yin, J.-H. Yang, J. Kang, Y. Yan, S.-H. Wei, J. Mater. Chem. A 3, 8926 (2015)

65. J. Bordas, J. Robertson, A. Jakobsson, J. Phys. C 11, 2607 (1978)

66. E. Doni, G. Grosso, G. Harbeke, E. Meier, E. Tosatti, Phys. Stat. Sol. (b) 68, 569 (1975)

67. Y. Yamada, T. Nakamura, M. Endo, A. Wakamiya, Y. Kanemitsu, Appl. Phys. Express 7, 032302 (2014)

68. A. Binek, F. C. Hanusch, P. Docampo, T. Bein, J. Phys. Chem. Lett. 6, 1249 (2015)

69. F. Hao, C. C. Stoumpos, Z. Liu, R. P. H. Chang, M. G. Kanatzidis, J. Am. Chem. Soc. 136, 16411 (2014)

70. O. N. Yunakova, V. K. Miloslavskii, E. N. Kovalenko, Opt. Spectrosc. 112, 91 (2012)

71. Y. Yamada, T. Nakamura, M. Endo, A. Wakamiya, Y. Kanemitsu, IEEE J. Photovoltaics 5, 401 (2015)

72. A. Miyata, A. Mitioglu, P. Plochocka, O. Portugall, J. T.-W. Wang, S. D. Stranks, H. J. Snaith, R. J. Nicholas, Nat. Phys. 11, 582 (2015)

73. E. Mosconi, A. Amat, M. K. Nazeeruddin, M. Grätzel, F. De Angelis, J. Phys. Chem. C 117, 13902 (2013)

74. F. Brivio, A. B. Walker, A. Walsh, APL mater. 1, 042111 (2013)

75. T. Umebayashi, K. Asai, T. Kondo, A. Nakao, Phys. Rev. B 67, 155405 (2003)

76. D. Li, J. Meng, Y. Niu, H. Zhao, C. Liang, Chem. Phys. Lett. 627, 13 (2015)

77. H. Fujiwara, S. Fujimoto, M. Tamakoshi, M. Kato, H. Kadowaki, T. Miyadera, H. Tampo, M. Chikamatsu, H. Shibata, Appl. Surf. Sci. doi: 10.1016/j.apsusc.2016.09.113 (2016)

78. M. P. Marder, Condensed Matter Physics (Wiley, Hoboken, 2010)

79. J. Even, L. Pedesseau, J.-M. Jancu, C. Katan, J. Phys. Chem. Lett. 4, 2999 (2013) 
80. P. Umari, E. Mosconi, F. De Angelis, Sci. Rep. 4, 4467 (2014)

81. F. Brivio, K. T. Butler, A. Walsh, M. van Schilfgaarde, Phys. Rev. B 89, 155204 (2014)

82. E. Menéndez-Proupin, P. Palacios, P. Wahnón, J. C. Conesa, Phys. Rev. B 90, 045207 (2014)

83. M. Marsman, J. Paier, A. Stroppa, G. Kresse, J. Phys. Condens. Matter 20, 064201 (2008)

84. C. Quarti, E. Mosconi, F. De Angelis, Chem. Mater. 26, 6557 (2014)

85. C. Motta, F. El-Mellouhi, S. Kais, N. Tabet, F. Alharbi, S. Sanvito, Nat. Commun. 6, $7026(2015)$

86. C. Motta, F. El-Mellouhi, S. Sanvito, Phys. Rev. B 93, 235412 (2016)

87. A. Nakane, H. Tampo, M. Tamakoshi, S. Fujimoto, K. M. Kim, S. Kim, H. Shibata, S. Niki, H. Fujiwara, J. Appl. Phys. 120, 064505 (2016)

88. J. H. Heo, D. H. Song, H. J. Han, S. Y. Kim, J. H. Kim, D. Kim, H. W. Shin, T. K. Ahn, C. Wolf, T.-W. Lee, S. H. Im, Adv. Mater. 27, 3424 (2015)

89. J. Shi, J. Dong, S. Lv, Y. Xu, L. Zhu, J. Xiao, X. Xu, H. Wu, D. Li, Y. Luo, Q. Meng, Appl. Phys. Lett. 104, 063901 (2014)

90. L. Etgar, P. Gao, Z. Xue, Q. Peng, A. K. Chandiran, B. Liu, Md. K. Nazeeruddin, M. Grätzel, J. Am. Chem. Soc. 134, 17396 (2012)

91. H. Zhou, Y. Shi, Q. Dong, H. Zhang, Y. Xing, K. Wang, Y. Du, T. Ma, J. Phys. Chem. Lett. 5, 3241 (2014)

92. W. Tress, N. Marinova, O. Inganäs, M. K. Nazeeruddin, S. M. Zakeeruddin, M. Graetzel, Adv. Energy Mater. 5, 1400812 (2014)

93. A. R. B. M. Yuoff, M. K. Nazeeruddin, J. Phys. Chem. Lett. 7, 851 (2016)

94. E. Edri, S. Kirmayer, A. Henning, S. Mukhopadhyay, K. Gartsman, Y. Rosenwaks, G. Hodes, D. Cahen, Nano Lett. 14, 1000 (2014)

95. V. W. Bergmann, S. A. L. Weber, F. J. Ramos, M. K. Nazeeruddin, M. Grätzel, D. Li, A. L. Domanski, I. Lieberwirth, S. Ahmad, R. Berger, Nat. Commun. 5, 5001 (2014)

96. W. Tress, N. Marinova, T. Moehl, S. M. Zakeeruddin, M. K. Nazeeruddin, M. Grätzel, Energy Environ. Sci. 8, 995 (2015)

97. Y. Zhang, M. Liu, G. E. Eperon, T. C. Leijtens, D. McMeekin, M. Saliba, W. Zhang, M. de Bastiani, A. Petrozza, L. M. Herz, M. B. Johnston, H. Lin, H. J. Snaith, Mater. Horiz. 2, 315 (2015)

98. E. Edri, S. Kirmayer, S. Mukhopadhyay, K. Gartsman, G. Hodes, D. Cahen, Nat. Commun. 5, 3461 (2014)

99. Q. Wang, Y. Shao, H. Xie, L. Lyu, X. Liu, Y. Gao, J. Huang, Appl. Phys. Lett. 105, 
$163508(2014)$

100. E. J. Juarez-Perez, M. Wußler, F. Fabregat-Santiago, K. Lakus-Wollny, E. Mankel, T. Mayer, W. Jaegermann, I. Mora-Sero, J. Phys. Chem. Lett. 5, 680 (2014)

101. W.-J. Yin, T. Shi, Y. Yan, Appl. Phys. Lett. 104, 063903 (2014)

102. J. Kim, S.-H. Lee, J. H. Lee, K.-H. Hong, J. Phys. Chem. Lett. 5, 1312 (2014)

103. M. L. Agiorgousis, Y.-Y. Sun, H. Zeng, S. Zhang, J. Am. Chem. Soc. 136, 14570 (2014)

104. Q. Chen, H. Zhou, T.-B. Song, S. Luo, Z. Hong, H.-S. Duan, L. Dou, Y. Liu, Y. Yang, Nano Lett. 14, 4158 (2014)

105. H. D. Kim, H. Ohkita, H. Benten, S. Ito, Adv. Mater. 28, 917 (2016)

106. K. Ghimire, A. Cimaroli, F. Hong, T. Shi, N. Podraza, Y. Yan, Proceedings of the $42^{\text {nd }}$ Photovoltaic Specialists Conference (2015) p.1

107. K. Ghimire, D. Zhao, A. Cimaroli, W. Ke, Y. Yan, N. J. Podraza, J. Phys. D 49, 405102 (2016)

108. L. Karki Gautam, H. Haneef, M. M. Junda, D. B. Saint John, N. J. Podraza, Thin Solid Films 571, 548 (2014)

109. K. Ghimire, D. Zhao, A. Cimaroli, W. Ke, M. Junda, Y. Yan, N. Podraza, Proceedings of the $43^{\text {rd }}$ Photovoltaic Specialists Conference (2016) p.89

110. Z. Song, S. C. Watthage, A. B. Phillips, B. L. Tompkins, R. J. Ellingson, M. J. Heben, Chem. Mater. 27, 4612 (2015)

111. P. Docampo T. Bein, Acc. Chem. Res. 49, 339 (2016)

112. C. Müller, T. Glaser, M. Plogmeyer, M. Sendner, S. Döring, A. A. Bakulin, C. Brzuska, R. Scheer, M. S. Pshenichnikov, W. Kowalsky, A. Pucci, R. Lovrinčić, Chem. Mater. 27, 7835 (2015)

113. I. Deretzis, A. Alberti, G. Pellegrino, E. Smecca, F. Giannazzo, N. Sakai, T. Miyasaka, A. La Magna, Appl. Phys. Lett. 106, 131904 (2015)

114. F. Matsumoto, S. M. Vorpahl, J. Q. Banks, E. Sengupta, D. S. Ginger, J. Phys. Chem. C 119, 20810 (2015) 